\title{
A Local-in-Time Theory for Singular SDEs with Applications to Fluid Models with Transport Noise
}

\author{
Diego Alonso-Orán ${ }^{1} \cdot$ Christian Rohde ${ }^{2} \cdot$ Hao Tang ${ }^{3}$
}

Received: 3 April 2021 / Accepted: 11 September 2021 / Published online: 17 October 2021

(C) The Author(s) 2021

\begin{abstract}
We establish a local theory, i.e., existence, uniqueness and blow-up criterion, for a general family of singular SDEs in Hilbert spaces. The key requirement relies on an approximation property that allows us to embed the singular drift and diffusion mappings into a hierarchy of regular mappings that are invariant with respect to the Hilbert space and enjoy a cancellation property. Various nonlinear models in fluid dynamics with transport noise belong to this type of singular SDEs. By establishing a cancellation estimate for certain differential operators of order one with suitable coefficients, we give the detailed constructions of such regular approximations for certain examples. In particular, we show novel local-in-time results for the stochastic two-component Camassa-Holm system and for the stochastic Córdoba-CórdobaFontelos model.
\end{abstract}

Keywords Singular stochastic differential equations - Stochastic fluid models . Transport noise

Communicated by Leslie Smith.

Alonso-Orán and H. Tang kindly acknowledge support by the Alexander von Humboldt Foundation. C. Rohde acknowledges support by Deutsche Forschungsgemeinschaft (DFG, German Research Foundation) under Germany's Excellence Strategy—EXC 2075-39074001.

$凶$ Hao Tang

haot@math.uio.no

Diego Alonso-Orán

alonso@iam.uni-bonn.de

Christian Rohde

christian.rohde@mathematik.uni-stuttgart.de

1 Institut für Angewandte Mathematik, Universität Bonn, Endenicher Allee 60, 53115 Bonn, Germany

2 Institut für Angewandte Analysis und Numerische Simulation, Universität Stuttgart, Pfaffenwaldring 57, 70569 Stuttgart, Germany

3 Department of Mathematics, University of Oslo, P.O. Box 1053, Blindern, 0316 Oslo, Norway 
Mathematics Subject Classification Primary 60H15 - 35Q35; Secondary 60H17 . 35A01

\section{Introduction}

Consider the initial value problem for a stochastic differential equation (SDE) with unknown process $X=X(t), t \geq 0$, given by

$$
\mathrm{d} X=(b(t, X)+g(t, X)) \mathrm{d} t+h(t, X) \mathrm{d} \mathcal{W}, \quad X(0)=X_{0} \in \mathcal{X}
$$

and let $\mathcal{X}, \mathcal{Y}$ and $\mathcal{Z}$ be three separable Hilbert spaces such that $\mathcal{X} \subset \mathcal{Y} \subset \mathcal{Z}$. Here, $\mathcal{W}$ denotes a cylindrical Wiener process defined on some separable Hilbert space $\mathbb{U}$; the drift is given by the sum of the mappings $b:[0, \infty) \times \mathcal{X} \rightarrow \mathcal{X}$ and $g:[0, \infty) \times \mathcal{X} \rightarrow$ $\mathcal{Z}$. The operator $h:[0, \infty) \times \mathcal{X} \rightarrow \mathcal{L}_{2}(\mathbb{U} ; \mathcal{Y})$ stands for the diffusion coefficient with $\mathcal{L}_{2}(\mathbb{U} ; \mathcal{Y})$ being the space of Hilbert-Schmidt operators from $\mathbb{U}$ to $\mathcal{Y}$. We call $(1.1)$ a singular initial value problem because $g$ and $h$ map $\mathcal{X}$ to the larger spaces $\mathcal{Z}$ and $\mathcal{Y}$, i.e., they are not invariant in $\mathcal{X}$. We refer to Sects. 2.1 and 2.2 for the precise setting.

In the fully regular case $\mathcal{X}=\mathcal{Y}=\mathcal{Z}$, it is well known that (local) Lipschitz conditions on $b(t, \cdot)+g(t, \cdot)$ and $h(t, \cdot)$ ensure that (1.1) admits unique (local) pathwise solution in $\mathcal{X}$. If additional monotonicity properties on the coefficients are imposed, then the Itô formula for Gelfand-triple Hilbert spaces can be exploited to assure global existence and continuity of solutions, cf. Kallianpur et al. (1995), Leha and Ritter (1985), Krylov and Rozovskiǔ (1979), Prévôt and Röckner (2007) and the references therein. Notably this covers also the case when the Hilbert spaces form a Gelfand triple.

In this work, we are interested in the singular scenario which appears in particular in the study of ideal fluid models. Indeed, when considering particular examples in Sobolev spaces $\mathcal{X}=H^{s}$, if $g(t, X)$ and $h(t, X)$ involve $\nabla X$ or some derivatives of $X$ (see our examples (3.7) and (3.11)), then $g(t, X)$ and $h(t, X)$ can not be expected to be in $\mathcal{X}=H^{s}$, either. Moreover, working with the abstract framework in (1.1) entails another difficulty compared to the regular or the Gelfand-triple case: the Itô formula is no longer available. To highlight the latter difficulty, let us recall the classical Itô formula for a Gelfand triplet $V \hookrightarrow H \hookrightarrow V^{*}$, where $H$ is a separable Hilbert space with inner product $(\cdot, \cdot)$ and $H^{*}$ is its dual; $V$ is a Banach space such that the embedding $V \hookrightarrow H$ is dense; $\mathcal{L}_{2}(\mathbb{U} ; H)$ is the space of Hilbert-Schmidt operators mapping $\mathbb{U}$ to $H$. Then, the following result is classical, see Krylov and Rozovskiu (1979, Theorem I.3.1) or Prévôt and Röckner (2007, Theorem 4.2.5).

Assume that $\mathbf{U}$ is a continuous $V^{*}$-valued stochastic process given by

$$
\mathbf{U}(t)=\mathbf{U}(0)+\int_{0}^{t} \mathbf{g}(s) \mathrm{d} s+\int_{0}^{t} \mathbf{G}(s) \mathrm{d} \mathcal{W}(s), \quad t \in[0, T]
$$

where $\mathbf{G} \in L^{2}\left(\Omega \times[0, T] ; \mathcal{L}_{2}(\mathbb{U} ; H)\right)$ and $\mathbf{g} \in L^{2}\left(\Omega \times[0, T] ; V^{*}\right)$ are both progressively measurable and $\mathbf{U}(0) \in L^{2}(\Omega ; H)$ is $\mathcal{F}_{0}$-measurable. If $\mathbf{U} \in$ $L^{2}(\Omega \times[0, T] ; V)$, then $\mathbf{U}$ is an $H$-valued continuous stochastic process and the 
Itô formula

$$
\begin{aligned}
\|\mathbf{U}(t)\|_{H}^{2}= & \|\mathbf{U}(0)\|_{H}^{2}+2 \int_{0}^{t} V^{*}\langle\mathbf{g}(s), \mathbf{U}(s)\rangle_{V} \mathrm{~d} s \\
& +2 \int_{0}^{t}(\mathbf{G}(s) \mathrm{d} \mathcal{W}, \mathbf{U}(s))_{H}+\int_{0}^{t}\|\mathbf{G}(s)\|_{\mathcal{L}_{2}(\mathbb{U} ; H)}^{2} \mathrm{~d} s .
\end{aligned}
$$

holds true $\mathbb{P}-$ a.s. for all $t \in[0, T]$.

We notice that (1.2) is applicable for $\mathbf{U}(0) \in H, \mathbf{U} \in L^{2}(\Omega \times[0, T] ; V), \mathbf{g} \in$ $L^{2}\left(\Omega \times[0, T] ; V^{*}\right)$ and $\mathbf{G} \in L^{2}\left(\Omega \times[0, T] ; \mathcal{L}_{2}(\mathbb{U} ; H)\right)$. However, if $\mathbf{G}$ is singular (not invariant in $H$ ), then $\mathbf{G} \in \mathcal{L}_{2}(\mathbb{U} ; H)$ is ambiguous. Besides, even though $\mathbf{g}$ is allowed to be less regular, (1.2) requires $\mathbf{U}(t)$ to be more regular than $\mathbf{U}(0)$, i.e., $\mathbf{U} \in V \hookrightarrow H \ni \mathbf{U}(0)$. In many cases (for example, stochastic ideal fluid models), we do not know that this holds true. Hence, (1.2) is not applicable in singular cases. Likewise, the concept of monotonicity cannot be applied, and the time continuity of the solution cannot be obtained directly, either.

The first major goal of this paper is to establish a local-in-time theory for (1.1) generalizing classical results for, e.g., the completely regular case $\mathcal{X}=\mathcal{Y}=\mathcal{Z}$. The second goal of this work is to show that the abstract theory for (1.1) can be used to establish new results for ideal fluid systems with transport noise.

1. To achieve the first goal, we fix in Sect. 2.2 the precise assumptions on the regular drift $b$ and in particular on the singular drift $g$ and diffusion $h$ (see Assumptions (A) and (B)). Then, we provide our main results for (1.1), including the existence, uniqueness, time regularity and a result characterizing the possible blow-up of pathwise solutions (see Theorem 2.1). The key requirements for the proof are the assumption on the existence of appropriate Lipschitz-continuous and monotone regularizations for the singular mappings. This allows us to exploit Itô-like formulas as above.

2. With the abstract framework at hand, we are able to construct such regular approximation schemes by using mollifying operators and establishing a cancellation property for certain differential operators (cf. Lemma A.5). The latter property has already been used in Crisan et al. (2019) in a somewhat different formulation to establish local-in-time results for the three dimensional Euler equations with transport noise. To set the stage, in Sect. 3, we consider two models governing ideal flows with particularly interesting stochastic perturbation, namely

- the two-component Camassa-Holm $(\mathrm{CH})$ system with transport noise (Holm and Luesink 2021), see (3.4),

- a nonlinear transport equation with non-local velocity, referred as the CórdobaCórdoba-Fontelos (CCF) model (Córdoba et al. 2005), with transport noise, see (3.10).

In both cases, we obtain a local-in-time theory in the sense of the abstractframework Theorem 2.1. The statements of our results are found in Sect. 3.2. To the best of our knowledge, they are the first results on well-posedness for these models. Finally, we explain in Sect. 3.5 how our abstract framework and the reg- 
ular approximation schemes can be applied to a broader class of fluid dynamics equations including the surface quasi-geostrophic (SQG) equation with transport noise.

\section{An Abstract Framework for a Class of Singular SDEs}

\subsection{Notations and Definitions}

To begin with, we introduce some notations. We consider a probability space $(\Omega, \mathcal{F}, \mathbb{P})$, where $\mathbb{P}$ is a probability measure on $\Omega$ and $\mathcal{F}$ is a $\sigma$-algebra. We endow the probability space $(\Omega, \mathcal{F}, \mathbb{P})$ with an increasing filtration $\left\{\mathcal{F}_{t}\right\}_{t \geq 0}$, which is a rightcontinuous filtration on $(\Omega, \mathcal{F})$ such that $\left\{\mathcal{F}_{0}\right\}$ contains all the $\mathbb{P}$-negligible subsets. For some separable Hilbert space $\mathbb{U}$ with a complete orthonormal basis $\left\{e_{i}\right\}_{i \in \mathbb{N}}$ the noise $\mathcal{W}$ in (1.1) is a cylindrical Wiener process, i.e., it is defined by

$$
\mathcal{W}=\sum_{k=1}^{\infty} W_{k} e_{k} \mathbb{P}-\text { a.s },
$$

where $\left\{W_{k}\right\}_{k \geq 1}$ is a sequence of mutually independent standard 1-D Brownian motions. To guarantee the convergence of the above formal summation, we consider a larger separable Hilbert space $\mathbb{U}_{0}$ such that the canonical injection $\mathbb{U} \hookrightarrow \mathbb{U}_{0}$ is HilbertSchmidt. Therefore, for any $T>0$, we have, cf. Da Prato and Zabczyk (2014), Gawarecki and Mandrekar (2011) and Karczewska (1998),

$$
\mathcal{W} \in C\left([0, T], \mathbb{U}_{0}\right) \mathbb{P}-\text { a.s. }
$$

Note that the choice of the auxiliary Hilbert spaces $\mathbb{U}$ and $\mathbb{U}_{0}$ is not crucial for our analysis. Thus, we let $\mathbb{U}$ and $\mathbb{U}_{0}$ be arbitrary but fixed in the sequel.

For some time $t>0$, the family $\sigma\left\{x_{1}(\tau), \ldots, x_{n}(\tau)\right\}_{\tau \in[0, t]}$ stands for the completion of the union $\sigma$-algebra generated by $\left(x_{1}(\tau), \ldots, x_{n}(\tau)\right)$ for $\tau \in[0, t]$. $\mathbb{E} Y$ stands for the mathematical expectation of a random variable $Y$ with respect to $\mathbb{P}$. From now on $\mathcal{S}=\left(\Omega, \mathcal{F}, \mathbb{P},\left\{\mathcal{F}_{t}\right\}_{t \geq 0}, \mathcal{W}\right)$ is called a stochastic basis.

For any Hilbert space $\mathbb{H}$ the inner product is denoted by $(\cdot, \cdot) \mathbb{H}$. Furthermore, the space $\mathcal{L}_{2}(\mathbb{U} ; \mathbb{H})$ contains all Hilbert-Schmidt operators $Z: \mathbb{U} \rightarrow \mathbb{H}$ with finite norm $\|Z\|_{\mathcal{L}_{2}(\mathbb{U} ; \mathbb{H})}^{2}=\sum_{k=1}^{\infty}\left\|Z e_{k}\right\|_{\mathbb{H}}^{2}$. As in Breit et al. (2018, Theorem 2.3.1), we see that for an $\mathbb{H}$-valued progressively measurable stochastic process $Z$ with $Z \in L^{2}\left(\Omega ; L_{\text {loc }}^{2}\left([0, \infty) ; \mathcal{L}_{2}(\mathbb{U} ; \mathbb{H})\right)\right)$, one can define the Itô stochastic integral

$$
\int_{0}^{t} Z \mathrm{~d} \mathcal{W}=\sum_{k=1}^{\infty} \int_{0}^{t} Z e_{k} \mathrm{~d} W_{k}
$$


Most notably for the analysis here, if $Z \in \mathcal{L}_{2}(\mathbb{U} ; \mathbb{H})$ and $\mathcal{W}$ is given as above, we have the Burkholder-Davis-Gundy (BDG) inequality

$$
\mathbb{E}\left(\sup _{t \in[0, T]}\left\|\int_{0}^{t} Z \mathrm{~d} \mathcal{W}\right\|_{\mathbb{H}}^{p}\right) \leq C \mathbb{E}\left(\int_{0}^{T}\|Z\|_{\mathcal{L}_{2}(\mathbb{U} ; \mathbb{H})}^{2} \mathrm{~d} t\right)^{\frac{p}{2}}, \quad p \geq 1,
$$

or in terms of the coefficients,

$$
\mathbb{E}\left(\sup _{t \in[0, T]}\left\|\sum_{k=1}^{\infty} \int_{0}^{t} Z e_{k} \mathrm{~d} W_{k}\right\|_{\mathbb{H}}^{p}\right) \leq C \mathbb{E}\left(\int_{0}^{T} \sum_{k=1}^{\infty}\left\|Z e_{k}\right\|_{\mathbb{H}}^{2} \mathrm{~d} t\right)^{\frac{p}{2}}, \quad p \geq 1 .
$$

Let $\mathbb{X}$ be a separable Banach space. $\mathcal{B}(\mathbb{X})$ denotes the Borel sets of $\mathbb{X}$ and $\mathscr{P}(\mathbb{X})$ stands for the collection of Borel probability measures on $(\mathbb{X}, \mathcal{B}(\mathbb{X}))$. We denote $\mathscr{P}_{r}(\mathbb{X})$ the family of probability measures in $\mathscr{P}(\mathbb{X})$ with finite moment of order $r \in[1, \infty)$, i.e., $\mathscr{P}_{r}(\mathbb{X})=\left\{\mu: \int_{\mathbb{X}}\|x\|_{\mathbb{X}}^{r} \mu(\mathrm{d} x)<\infty\right\}$. For two Banach spaces $\mathbb{X}$ and $\mathbb{Y}, \mathbb{X} \hookrightarrow \mathbb{Y}$ means that $\mathbb{X}$ is embedded continuously into $\mathbb{Y}$, and $\mathbb{X} \hookrightarrow \hookrightarrow \mathbb{Y}$ means that the embedding is compact. For a set $E, \mathbf{1}_{E}$ denotes the indicator function on $E$.

Next, let us make precise two different notions of solutions in the Hilbert space $\mathcal{X}$ for the Cauchy problem (1.1).

Definition 2.1 (Martingale solutions) Let $\mu_{0} \in \mathscr{P}(\mathcal{X})$. A triple $(\mathcal{S}, X, \tau)$ is said to be a martingale solution to (1.1) if

1. $\mathcal{S}=\left(\Omega, \mathcal{F}, \mathbb{P},\left\{\mathcal{F}_{t}\right\}_{t \geq 0}, \mathcal{W}\right)$ is a stochastic basis and $\tau$ is a stopping time with respect to $\left\{\mathcal{F}_{t}\right\}_{t \geq 0}$

2. $X(\cdot \wedge \tau): \Omega \times[0, \infty) \rightarrow \mathcal{X}$ is an $\mathcal{F}_{t}$-progressively measurable process such that it is continuous in $\mathcal{Z}, \mu_{0}(\cdot)=\mathbb{P}\left\{X_{0} \in \cdot\right\}$ for all $\cdot \in \mathcal{B}(\mathcal{X})$ and for every $t>0$,

$$
X(t \wedge \tau)-X_{0}=\int_{0}^{t \wedge \tau}\left(b\left(t^{\prime}, X\left(t^{\prime}\right)\right)+g\left(t^{\prime}, X\left(t^{\prime}\right)\right)\right) \mathrm{d} t^{\prime}+\int_{0}^{t \wedge \tau} h\left(t^{\prime}, X\left(t^{\prime}\right)\right) \mathrm{d} \mathcal{W} \mathbb{P}-\text { a.s. }
$$

In (2.2), $\int_{0}^{\cdot}\left\{b\left(t^{\prime}, X\left(t^{\prime}\right)\right)+g\left(t^{\prime}, X\left(t^{\prime}\right)\right)\right\} \mathrm{d} t^{\prime}$ is the Bochner integral on $\mathcal{Z}$ and $\int_{0} h\left(t^{\prime}, X\left(t^{\prime}\right)\right) \mathrm{d} \mathcal{W}$ is a continuous local martingale on $\mathcal{Y}$.

3. If $\tau=\infty \mathbb{P}-$ a.s., then we say that the martingale solution is global.

The stronger concept of pathwise solutions is provided in

Definition 2.2 (Pathwise solutions) Let $\mathcal{S}=\left(\Omega, \mathcal{F}, \mathbb{P},\left\{\mathcal{F}_{t}\right\}_{t \geq 0}, \mathcal{W}\right)$ be a fixed stochastic basis. Let $X_{0}$ be an $\mathcal{X}$-valued $\mathcal{F}_{0}$-measurable random variable. A local pathwise solution to (1.1) is a pair $(X, \tau)$, where $\tau$ is a stopping time satisfying $\mathbb{P}\{\tau>0\}=1$ and $X: \Omega \times[0, \tau] \rightarrow \mathcal{X}$ is an $\mathcal{F}_{t}$-progressively measurable process satisfying $(2.2)$ and $X(\cdot \wedge \tau) \in C([0, \infty) ; \mathcal{X})$ almost surely.

It follows from Definition 2.1 that, if a martingale solution exists, then (2.2) implies that

$$
\int_{0}^{t \wedge \tau}\left(b\left(t^{\prime}, X\left(t^{\prime}\right)\right)+g\left(t^{\prime}, X\left(t^{\prime}\right)\right)\right) \mathrm{d} t^{\prime}+\int_{0}^{t \wedge \tau} h\left(t^{\prime}, X\left(t^{\prime}\right)\right) \mathrm{d} \mathcal{W}
$$


takes values in $\mathcal{X}$, even though $g$ and $h$ are not invariant in $\mathcal{X}$. Moreover, Definition 2.2 implies that if a pathwise solution exists, then (2.3) is continuous in time in $\mathcal{X}$.

To study the possible blow-up of the solutions, we need the following concept of maximal solutions.

Definition 2.3 (Maximal solutions) Let $\mathcal{S}=\left(\Omega, \mathcal{F}, \mathbb{P},\left\{\mathcal{F}_{t}\right\}_{t \geq 0}, \mathcal{W}\right)$ be a fixed stochastic basis. Let $X_{0}$ be an $\mathcal{X}$-valued $\mathcal{F}_{0}$-measurable random variable. $\left(X, \tau^{*}\right)$ is called a maximal pathwise solution to (1.1) if there is an increasing sequence $\tau_{n} \rightarrow \tau^{*}$ such that for any $n \in \mathbb{N},\left(X, \tau_{n}\right)$ is a pathwise solution satisfying

$$
\sup _{t \in\left[0, \tau_{n}\right]}\|X\| \mathcal{X} \geq n \text { a.e. on }\left\{\tau^{*}<\infty\right\} \text {. }
$$

Particularly, if $\tau^{*}=\infty$ almost surely, then such a solution is called global.

\subsection{Assumptions and Main Results}

To study the existence of martingale and pathwise solutions, we need the following assumptions on the three separable Hilbert spaces $\mathcal{X}, \mathcal{Y}, \mathcal{Z}$ and on the coefficients $b$, $g$ and $h$ in (1.1). Recall that $\left\{e_{i}\right\}_{i \in \mathbb{N}}$ is a complete orthonormal basis of $\mathbb{U}$.

We first impose some conditions guaranteeing the existence of martingale solutions.

Assumption (A) The Hilbert spaces satisfy the embedding relation $\mathcal{X} \hookrightarrow \mathcal{Y} \hookrightarrow \hookrightarrow \mathcal{Z}$ and the coefficients $b:[0, \infty) \times \mathcal{X} \rightarrow \mathcal{X}, g:[0, \infty) \times \mathcal{X} \rightarrow \mathcal{Z}$ and $h:[0, \infty) \times \mathcal{X} \rightarrow$ $\mathcal{L}_{2}(\mathbb{U} ; \mathcal{Y})$ are continuous in both variables. Let $\mathcal{V}$ be a Banach space satisfying $\mathcal{Z} \hookrightarrow \mathcal{V}$. There are non-decreasing locally bounded functions $f(\cdot), k(\cdot), q(\cdot) \in C$ $([0,+\infty) ;[0,+\infty))$ such that the following conditions hold true.

$\left(A_{1}\right)$ For all $(t, X) \in[0, \infty) \times \mathcal{X}$, we have

$$
\|b(t, X)\| \mathcal{X} \leq k(t) f(\|X\| \mathcal{V})\|X\| \mathcal{X}
$$

and for all $N \in \mathbb{N}$,

$$
\sup _{\|X\|_{\mathcal{X}},\|Y\|_{\mathcal{X} \leq N}}\left\{\mathbf{1}_{\{X \neq Y\}} \frac{\|b(t, X)-b(t, Y)\| \mathcal{X}}{\|X-Y\| \mathcal{X}}\right\} \leq q(N) k(t) .
$$

Besides, for any bounded sequence $\left\{X_{\varepsilon}\right\} \subset \mathcal{X}$ such that $X_{\varepsilon} \rightarrow X$ in $\mathcal{Z}$,

$$
\lim _{n \rightarrow \infty}\left\|b\left(t, X_{\varepsilon}\right)-b(t, X)\right\|_{\mathcal{Z}}=0, t \geq 0
$$

$\left(\boldsymbol{A}_{2}\right)$ For $\varepsilon \in(0,1)$ and $N \geq 1$ there exist regular maps

$$
g_{\varepsilon}:[0, \infty) \times \mathcal{X} \rightarrow \mathcal{X}, \quad h_{\varepsilon}:[0, \infty) \times \mathcal{X} \rightarrow \mathcal{L}_{2}(\mathbb{U} ; \mathcal{X})
$$


and constants $C_{\varepsilon, N}>0$ such that for all $t \geq 0$ the bounds

$$
\begin{gathered}
\sup _{\varepsilon \in(0,1),\|X\|_{\mathcal{X} \leq N}}\left\{\left\|g_{\varepsilon}(t, X)\right\|_{\mathcal{Z}}+\|g(t, X)\|_{\mathcal{Z}}+\left\|h_{\varepsilon}(t, X)\right\|_{\mathcal{L}_{2}(\mathbb{U} ; \mathcal{Y})}\right. \\
+\|h(t, X)\|_{\left.\mathcal{L}_{2}(\mathbb{U} ; \mathcal{Y})\right\} \leq q(N) k(t),} \\
\sup _{\|X\|_{\mathcal{X} \leq N}}\left\{\left\|g_{\varepsilon}(t, X)\right\| \mathcal{X}+\left\|h_{\varepsilon}(t, X)\right\|_{\left.\mathcal{L}_{2}(\mathbb{U} ; \mathcal{X})\right\} \leq C_{\varepsilon, N} k(t),}\right.
\end{gathered}
$$

and

$\sup _{\|X\|_{\mathcal{X}},\|Y\|_{\mathcal{X}} \leq N}\left\{\mathbf{1}_{\{X \neq Y\}}\left(\frac{\left\|g_{\varepsilon}(t, X)-g_{\varepsilon}(t, Y)\right\|_{\mathcal{X}}}{\|X-Y\|_{\mathcal{X}}}+\frac{\left\|h_{\varepsilon}(t, X)-h_{\varepsilon}(t, Y)\right\|_{\mathcal{L}_{2}(\mathbb{U} ; \mathcal{X})}}{\|X-Y\|_{\mathcal{X}}}\right)\right\} \leq C_{\varepsilon, N} k(t)$

hold. Moreover, for any $T>0$ and any bounded sequence $\left\{X_{\varepsilon}(t)\right\} \subset$ $C([0, T] ; \mathcal{X})$ such that $X_{\varepsilon} \rightarrow X$ in $C([0, T] ; \mathcal{Z})$, we suppose

$$
\lim _{\varepsilon \rightarrow 0} \int_{0}^{t} \mid \mathcal{Z}\left\langle g_{\varepsilon}\left(t, X_{\varepsilon}\left(t^{\prime}\right)\right)-g\left(t, X\left(t^{\prime}\right)\right), \phi\left|\mathcal{Z}^{*}\right| \mathrm{d} t^{\prime}=0 \forall \phi \in \mathcal{Z}^{*}\right.
$$

and

$$
\lim _{n \rightarrow \infty}\left\|h_{\varepsilon}\left(t, X_{\varepsilon}\right)-h(t, X)\right\|_{\mathcal{L}_{2}(\mathbb{U} ; \mathcal{Z})}=0
$$

Here $\mathcal{Z}\langle\cdot, \cdot\rangle_{\mathcal{Z}^{*}}$ denotes the dual pairing in $\mathcal{Z}$.

$\left(\boldsymbol{A}_{3}\right)$ Let $g_{\varepsilon}$ and $h_{\varepsilon}$ be given in $\left(\mathbf{A}_{2}\right)$. For all $n \geq 1$ and $(t, X) \in[0, \infty) \times \mathcal{X}$, we have

$$
\sum_{i=1}^{\infty}\left|\left(h_{\varepsilon}(t, X) e_{i}, X\right) \mathcal{X}\right|^{2} \leq k(t) f(\|X\| \mathcal{V})\|X\|_{\mathcal{X}}^{4}
$$

and

$$
2\left(g_{\varepsilon}(t, X), X\right)_{\mathcal{X}}+\left\|h_{\varepsilon}(t, X)\right\|_{\mathcal{L}_{2}(\mathbb{U} ; \mathcal{X})}^{2} \leq k(t) f(\|X\| \mathcal{V})\|X\|_{\mathcal{X}}^{2}
$$

Remark 2.1 It is important to notice that Assumption (A) can be satisfied for singular mappings $b$ and $g$, since the constants $C_{\varepsilon, N}$ in Assumption (A) may be non-decreasing in $N$ for fixed $\varepsilon$ and may explode for $\varepsilon \rightarrow 0$ with $N$ fixed.

For pathwise solution, we will need more assumptions.

Assumption (B) To consider the existence, uniqueness and time continuity of a pathwise solution, we need the following assumptions:

$\left(\boldsymbol{B}_{1}\right)$ For any $t \geq 0$ and $N \geq 1$, we have

$$
\sup _{\|X\|_{\mathcal{X}},\|Y\|_{\mathcal{X} \leq N}}\left\{\mathbf{1}_{\{X \neq Y\}} \frac{\|b(t, X)-b(t, Y)\|_{\mathcal{Z}}}{\|X-Y\|_{\mathcal{Z}}}\right\} \leq q(N) k(t)
$$


and

$$
\sup _{\|X\|_{\mathcal{X}},\|Y\|_{\mathcal{X}} \leq N}\left\{\mathbf{1}_{\{X \neq Y\}} \frac{2(g(t, X)-g(t, Y), X-Y)_{\mathcal{Z}}+\|h(t, X)-h(t, Y)\|_{\mathcal{L}_{2}(\mathbb{U} ; \mathcal{Z})}^{2}}{\|X-Y\|_{\mathcal{Z}}^{2}}\right\} \leq q(N) k(t) .
$$

( $\left.\boldsymbol{B}_{2}\right)$ The embedding $\mathcal{X} \hookrightarrow \mathcal{Z}$ is dense, and there is a family of continuous linear operators $\left\{T_{\varepsilon}: \mathcal{Z} \rightarrow \mathcal{X}\right\}_{\varepsilon \in(0,1)}$ such that

$$
\left\|T_{\varepsilon} X\right\|_{\mathcal{X}} \leq\|X\|_{\mathcal{X}}, \quad \lim _{n \rightarrow \infty}\left\|T_{\varepsilon} X-X\right\|_{\mathcal{X}}=0, \quad X \in \mathcal{X}
$$

and for all $t \geq 0, N \geq 1$

$$
\begin{aligned}
& \sup _{\varepsilon \in(0,1),\|X\|_{\mathcal{X}} \leq N} 2\left(T_{\varepsilon} g(t, X), T_{\varepsilon} X\right) \mathcal{X}+\left\|T_{\varepsilon} h(t, X)\right\|_{\mathcal{L}_{2}(\mathbb{U} ; \mathcal{X}) \leq q(N) k(t),}^{2} \\
& \sup _{\varepsilon \in(0,1),\|X\|_{\mathcal{X} \leq N}} \sum_{i=1}^{\infty}\left|\left(T_{\varepsilon} h(t, X) e_{i}, T_{\varepsilon} X\right) \mathcal{X}\right|^{2} \leq q(N) k(t)
\end{aligned}
$$

hold.

Finally, we introduce the following assumption, which will be used to derive a blow-up criterion.

Assumption (C) There is a family of continuous linear operators $\left\{Q_{\varepsilon}: \mathcal{Z} \rightarrow \mathcal{X}\right\}_{\varepsilon \in(0,1)}$ such that (2.16) with $Q_{\varepsilon}$ replacing $T_{\varepsilon}$ and

$$
\begin{aligned}
& \sup _{\varepsilon \in(0,1)} \sum_{i=1}^{\infty}\left|\left(Q_{\varepsilon} h(t, X) e_{i}, Q_{\varepsilon} X\right) \mathcal{X}\right|^{2} \leq k(t) f\left(\|X\|_{\mathcal{V}}\right)\|X\|_{\mathcal{X}}^{2}\left\|Q_{\varepsilon} X\right\|_{\mathcal{X}}^{2}, \\
& \sup _{\varepsilon \in(0,1)} 2\left(Q_{\varepsilon} g(t, X), Q_{\varepsilon} X\right) \mathcal{X}+\left\|Q_{\varepsilon} h(t, X)\right\|_{\mathcal{L}_{2}(\mathbb{U} ; \mathcal{X})}^{2} \leq k(t) f(\|X\| \mathcal{V})\|X\|_{\mathcal{X}}^{2}
\end{aligned}
$$

hold true for $t \geq 0$.

Then, we can state our main results for the initial value problem (1.1):

Theorem 2.1 Let us consider the initial value problem (1.1). Then, we have that:

(i) Let Assumption (A) hold. Then, for any $\mu_{0} \in \mathscr{P}_{2}(\mathcal{X}),(1.1)$ has a local martingale solution $(\mathcal{S}, X, \tau)$ in the sense of Definition 2.1.

(ii) Let $\mathcal{S}=\left(\Omega, \mathcal{F}, \mathbb{P},\left\{\mathcal{F}_{t}\right\}_{t \geq 0}, \mathcal{W}\right)$ be a fixed stochastic basis. If Assumptions (A) and (B) hold, then for any $\mathcal{F}_{0}$-measurable random variable $X_{0} \in L^{2}(\Omega ; \mathcal{X})$, (1.1) has a local unique pathwise solution $(X, \tau)$, in the sense of Definition 2.2 such that

$$
X(\cdot \wedge \tau) \in L^{2}(\Omega ; C([0, \infty) ; \mathcal{X}))
$$


(iii) Let $\left(X, \tau^{*}\right)$ be the maximal solution to (1.1), in the sense of Definition 2.3, under Assumptions (A) and (B). If additionally Assumption (C) holds true, then X blows up in $\mathcal{X}$ if only and only if it blows up in $\mathcal{V}$, i.e.,

$$
\mathbf{1}_{\left\{\lim \sup _{t \rightarrow \tau^{*}}\|X\|_{\mathcal{X}}=\infty\right\}}=\mathbf{1}_{\left\{\lim \sup _{t \rightarrow \tau^{*}}\|X\|_{\mathcal{V}}=\infty\right\}} \mathbb{P}-\text { a.s. }
$$

Remark 2.2 We first remark that the singular terms $g$ and $h$ are in general not monotone in the sense of Pardoux (1972) and Prévôt and Röckner (2007). So, the well-known approximation scheme under a Gelfand triple developed for quasi-linear SPDEs does not work for the present model. Motivated by Tang (2018), Li et al. (2021) and Ren et al. (2020), we will employ a regularization argument to overcome this difficulty. Let us give some explanations on Assumptions (A), (B) and (C) that makes precise the required regularization procedure.

- Assumption $\left(\mathbf{A}_{1}\right)$ provides the local Lipschitz continuity for the regular drift coefficient $b(t, X)$ and bounds its growth. Assumption $\left(\mathbf{A}_{2}\right)$ requires the local Lipschitz continuity on the approximations $g_{\varepsilon}$ and $h_{\varepsilon}$ of the singular terms $g$ and $h$, which together with $\left(\mathbf{A}_{1}\right)$ will ensure local-in-time existence for some approximate problem. In Sect. 3.1 we will show how to construct such approximations using mollifiers.

- Condition (2.13) in Assumption $\left(\mathbf{A}_{3}\right)$ is a cancellation property. Even though $g$ and $h$ are not invariant in $\mathcal{X}$ (hence, $(g(t, X), X) \mathcal{X}$ and $\|h(t, X)\|_{\mathcal{L}_{2}(\mathbb{U} ; \mathcal{X})}$ may be infinite), we can formally require that $(g(t, X), X) \mathcal{X}+\|h(t, X)\|_{\mathcal{L}_{2}(\mathbb{U} ; \mathcal{X})}$ can be controlled. Hence, (2.13) can be viewed as a renormalization type condition in the sense that it specifies this relationship for $g_{\varepsilon}$ and $h_{\varepsilon}$ such that $\left(g_{\varepsilon}(t, X), X\right) \mathcal{X}$ and $\left\|h_{\varepsilon}(t, X)\right\|_{\mathcal{L}_{2}(\mathbb{U} ; \mathcal{X})}$ make sense.

- Since $g$ and $h$ are singular, we need $\left(\mathbf{B}_{1}\right)$ on the joint space $\mathcal{Z}$ to guarantee pathwise uniqueness.

- As explained in the introduction, we can not use the Itô formula (1.2) to obtain the time continuity of the solution directly. This is why we need to Assumptions $\left(\mathbf{B}_{2}\right)$ and $(\mathrm{C})$ to establish time continuity and blow-up criterion, respectively. Assumption $(C)$ is stronger than $\left(\mathbf{B}_{2}\right)$ because we need both, the validity of the Itô formula and the growth condition. However, the dense embedding $\mathcal{X} \hookrightarrow \mathcal{Z}$ is not necessary for deriving the blow-up criterion. Moreover, in applications, usually one can take $T_{\varepsilon}=Q_{\varepsilon}$.

- In view of Assumption (A), it is worthwhile noticing that the regular drift $b$ will not be used to control the singular terms, i.e., our result covers the case $b \equiv 0$, where both the drift and diffusion in (1.1) are singular. However, we assume that the problem (1.1) has a regular part to cover more ideal fluid models.

Remark 2.3 In Debussche et al. (2011), an abstract fluid model involving a Stokes operator (viscous term) and a regular noise coefficient is studied. The existence of martingale solution is shown under the condition that the initial measure has finite moment of order $r>8$ (see Debussche et al. 2011, Theorem 6.1). In the present work, we are able to treat inviscid fluid models with singular transport type noises and improve the initial measure requirement to $r=2$, i.e., $\mu_{0} \in \mathscr{P}_{2}(\mathcal{X})$ in (i) in Theorem 2.1. 
Remark 2.4 We also remark that when the noise coefficient $h(t, X)$ is as regular as the solution $X$ and the singularity of (1.1) only arises in $g$, namely $b:[0, \infty) \times \mathcal{X} \rightarrow \mathcal{X}$, $h:[0, \infty) \times \mathcal{X} \rightarrow \mathcal{L}_{2}(\mathbb{U} ; \mathcal{X})$ and $g:[0, \infty) \times \mathcal{X} \rightarrow \mathcal{Z}$, one can also obtain a local theory as in Theorem 2.1 even under weaker conditions as in Assumption (A).

\subsection{Proof of (i) in Theorem 2.1}

For the sake of clarity, we split the proof into the following subsections.

\subsubsection{Approximation Scheme and Uniform Estimates}

For $\mu_{0} \in \mathscr{P}_{2}(\mathcal{X})$, we first fix a stochastic basis $\mathcal{S}$ and a random variable $X_{0}$ such that the distribution law of $X_{0}$ is $\mu_{0}$. For any $R>1$, we let $\chi_{R}(x):[0, \infty) \rightarrow[0,1]$ be a $C^{\infty}$-function such that $\chi_{R}(x)=1$ for $x \in[0, R]$ and $\chi_{R}(x)=0$ for $x>2 R$. Then, we consider a cut-off version of (1.1) given by

$$
\left\{\begin{array}{l}
\mathrm{d} X=\chi_{R}^{2}\left(\|X\|_{\mathcal{V}}\right)[b(t, X)+g(t, X)] \mathrm{d} t+\chi_{R}\left(\|X\|_{\mathcal{V}}\right) h(t, X) \mathrm{d} \mathcal{W} \\
X(0)=X_{0}
\end{array}\right.
$$

We have not posed any structural properties like monotonicity on the singular mappings $g, h$ that ensure the existence of solutions for (2.23). Therefore, we employ the regular approximations $g_{\varepsilon}$ and $h_{\varepsilon}$ from Assumption (A) which leads us to the regular approximate version

$$
\left\{\begin{array}{l}
\mathrm{d} X=H_{1, \varepsilon}(t, X) \mathrm{d} t+H_{2, \varepsilon}(t, X) \mathrm{d} \mathcal{W} \\
H_{1, \varepsilon}(t, X)=\chi_{R}^{2}(\|X\| \mathcal{V})\left(b(t, X)+g_{\varepsilon}(t, X)\right) \\
H_{2, \varepsilon}(t, X)=\chi_{R}(\|X\| \mathcal{V}) h_{\varepsilon}(t, X) \\
X(0)=X_{0}
\end{array}\right.
$$

For (2.24) we can obtain the following global existence result.

Lemma 2.1 For $\mu_{0} \in \mathscr{P}_{2}(\mathcal{X})$, we fix a stochastic basis $\mathcal{S}$ and a $\mathcal{F}_{0}$-measurable random variable $X_{0}$ such that the distribution of $X_{0}$ is $\mu_{0}$. Let $R>1$ be fixed.

For each $\varepsilon \in(0,1)$, the problem (2.24) has a global solution $X_{\varepsilon}$. Moreover, for any sequence $\left\{\varepsilon_{n}\right\}_{n \in \mathbb{N}}$ and for any $T>0$, we have that

$$
v_{\varepsilon_{n}}(\cdot)=\mathbb{P}\left\{\left(X_{\varepsilon_{n}}, \mathcal{W}\right) \in \cdot\right\}
$$

defines a tight sequence in $\mathscr{P}\left(C([0, T] ; \mathcal{Z}) \times C\left([0, T] ; \mathbb{U}_{0}\right)\right)$.

Proof From $\left(\mathbf{A}_{1}\right),\left(\mathbf{A}_{2}\right)$, it is easy to see that for each $n \geq 1, H_{1, \varepsilon}(t, X)$ and $H_{2, \varepsilon}(t, X)$ are locally Lipschitz in $X \in \mathcal{X}$. Moreover, the growth of $\left\|H_{1, \varepsilon}(\cdot, X)\right\|_{\mathcal{X}}$ and $\left\|H_{2, \varepsilon}(\cdot, X)\right\|_{\mathcal{L}_{2}(\mathbb{U} ; \mathcal{X})}$ is controlled by the continuous function $k(t)$. Therefore, for each $\varepsilon \in(0,1)$, there is a stopping time $\tau_{\varepsilon}^{*}>0$ almost surely such that the problem (2.24) has a unique solution $X_{\varepsilon} \in L^{2}\left(\Omega ; C\left(\left[0, \tau_{\varepsilon}^{*}\right) ; \mathcal{X}\right)\right)$, see Leha and Ritter (1985) 
or Kallianpur et al. (1995, Theorem 5.1.1). Next, we prove that the solution is actually a global solution. To see this, we use the Itô formula in $\mathcal{X}$ for the regular mappings $g_{\varepsilon}, h_{\varepsilon}$ to find

$$
\begin{aligned}
\mathrm{d}\left\|X_{\varepsilon}\right\|_{\mathcal{X}}^{2}= & 2 \sum_{k=1}^{\infty} \chi_{R}\left(\left\|X_{\varepsilon}\right\|_{\mathcal{V}}\right)\left(h_{\varepsilon}\left(t, X_{\varepsilon}\right) e_{k}, X_{\varepsilon}\right)_{\mathcal{X}} \mathrm{d} W_{k}+2 \chi_{R}^{2}\left(\left\|X_{\varepsilon}\right\|_{\mathcal{V}}\right)\left(b\left(t, X_{\varepsilon}\right), X_{\varepsilon}\right)_{\mathcal{X}} \mathrm{d} t \\
& +2 \chi_{R}^{2}\left(\left\|X_{\varepsilon}\right\|_{\mathcal{V}}\right)\left(g_{\varepsilon}\left(t, X_{\varepsilon}\right), X_{\varepsilon}\right)_{\mathcal{X}} \mathrm{d} t+\chi_{R}^{2}\left(\left\|X_{\varepsilon}\right\|_{\mathcal{V}}\right)\left\|h_{\varepsilon}\left(t, X_{\varepsilon}\right)\right\|_{\mathcal{L}_{2}(\mathbb{U} ; \mathcal{X})}^{2} \mathrm{~d} t \\
& :=\sum_{k=1}^{\infty} J_{1, k} \mathrm{~d} W_{k}+\sum_{i=2}^{4} J_{i} \mathrm{~d} t
\end{aligned}
$$

For any $T>0$, we integrate (2.26), take a supremum for $t \in[0, T]$ and then use the BDG inequality, $\left(\mathbf{A}_{1}\right)$ and $\left(\mathbf{A}_{3}\right)$ to find a constant $C=C_{R}>0$ depending on $R$ such that

$$
\begin{aligned}
& \mathbb{E} \sup _{t \in[0, T]}\left\|X_{\varepsilon}\right\|_{\mathcal{X}}^{2}-\mathbb{E}\left\|X_{0}\right\|_{\mathcal{X}}^{2} \\
& \lesssim \mathbb{E}\left(\int_{0}^{T} \sum_{k=1}^{\infty} J_{1, k}^{2} \mathrm{~d} t\right)^{\frac{1}{2}}+\int_{0}^{T}\left|J_{2}\right| \mathrm{d} t+\int_{0}^{T}\left|J_{3}+J_{4}\right| \mathrm{d} t \\
& \lesssim \mathbb{E}\left(\int_{0}^{T} k(t) \chi_{R}^{2}\left(\left\|X_{\varepsilon}\right\| \mathcal{V}\right) f\left(\left\|X_{\varepsilon}\right\| \mathcal{V}\right)\left\|X_{\varepsilon}\right\|_{\mathcal{X}}^{4} \mathrm{~d} t\right)^{\frac{1}{2}} \\
& +\int_{0}^{T} k(t) \chi_{R}^{2}\left(\left\|X_{\varepsilon}\right\| \mathcal{V}\right) f\left(\left\|X_{\varepsilon}\right\| \mathcal{V}\right)\left\|X_{\varepsilon}\right\|_{\mathcal{X}}^{2} \mathrm{~d} t \\
& \leq C_{R} \mathbb{E}\left(\sup _{t \in[0, T]}\left\|X_{\mathcal{\varepsilon}}\right\|_{\mathcal{X}}^{2} \int_{0}^{T} k(t)\left\|X_{\mathcal{E}}\right\|_{\mathcal{X}}^{2} \mathrm{~d} t\right)^{\frac{1}{2}}+C_{R} \int_{0}^{T} k(t)\left\|X_{\mathcal{\varepsilon}}\right\|_{\mathcal{X}}^{2} \mathrm{~d} t \\
& \leq \frac{1}{2} \mathbb{E} \sup _{t \in[0, T]}\left\|X_{\mathcal{E}}\right\|_{\mathcal{X}}^{2}+C_{R} \int_{0}^{T} k(t) \mathbb{E} \sup _{t^{\prime} \in[0, t]}\left\|X_{\mathcal{\varepsilon}}\right\|_{\mathcal{X}}^{2} \mathrm{~d} t
\end{aligned}
$$

Via Grönwall's inequality, we arrive at the $\varepsilon$-independent bound

$$
\sup _{\varepsilon \in(0,1)} \mathbb{E} \sup _{t \in[0, T]}\left\|X_{\varepsilon}(t)\right\|_{\mathcal{X}}^{2} \leq C\left(R, X_{0}, T\right)
$$

Since $T>0$ can be chosen arbitrarily, we see in particular that $X_{\varepsilon}$ is a global solution for each $\varepsilon \in(0,1)$.

Moreover, the bound (2.27) implies that the stopping times

$$
\tau_{N}^{\varepsilon}:=\inf \left\{t \geq 0: \sup _{t^{\prime} \in[0, t]}\left\|X_{\varepsilon}\right\| \mathcal{X} \geq N\right\}, N \geq 1, \varepsilon \in(0,1)
$$


satisfy

$$
\mathbb{P}\left(\tau_{N}^{\varepsilon}<T\right) \leq \mathbb{P}\left(\sup _{t \in[0, T]}\left\|X_{\varepsilon}\right\| \mathcal{X} \geq N\right) \leq \frac{C\left(R, X_{0}, T\right)}{N^{2}}
$$

Now we turn to prove the tightness result on the Borel measure in (2.25). For any given $\delta \in(0,1)$, we get that

$$
\begin{aligned}
& \mathbb{E} \sup _{\left[t_{1}, t_{2}\right] \subset[0, T], t_{2}-t_{1}<\delta}\left(1 \wedge\left\|X_{\mathcal{E}}\left(t_{2}\right)-X_{\mathcal{E}}\left(t_{1}\right)\right\|_{\mathcal{Z}}\right) \\
& \quad \leq \mathbb{E}\left(\sup _{\left[t_{1}, t_{2}\right] \subset[0, T], t_{2}-t_{1}<\delta}\left(1 \wedge\left\|X_{\mathcal{E}}\left(t_{2}\right)-X_{\mathcal{E}}\left(t_{1}\right)\right\|_{\mathcal{Z}}\right) \mathbf{1}_{\left\{\tau_{N}^{\varepsilon}<T\right\}}\right) \\
& \quad+\mathbb{E}\left(\sup _{\left[t_{1}, t_{2}\right] \subset[0, T], t_{2}-t_{1}<\delta}\left(1 \wedge\left\|X_{\varepsilon}\left(t_{2}\right)-X_{\mathcal{E}}\left(t_{1}\right)\right\|_{\mathcal{Z}}\right) \mathbf{1}_{\left\{\tau_{N}^{\varepsilon} \geq T\right\}}\right) \\
& \quad \leq \mathbb{P}\left\{\tau_{N}^{\varepsilon}<T\right\}+\mathbb{E}\left(\sup _{\left[t_{1}, t_{2}\right] \subset\left[0, T \wedge \tau_{N}^{\varepsilon}\right], t_{2}-t_{1}<\delta}\left(1 \wedge\left\|X_{\mathcal{E}}\left(t_{2}\right)-X_{\mathcal{E}}\left(t_{1}\right)\right\|_{\mathcal{Z}}\right) \mathbf{1}_{\left\{\tau_{N}^{\varepsilon} \geq T\right\}}\right) \\
& \quad \leq \frac{C\left(R, X_{0}, T\right)}{N^{2}}+\mathbb{E}\left(\sup _{\left[t_{1}, t_{2}\right] \subset\left[0, T \wedge \tau_{N}^{\varepsilon}\right], t_{2}-t_{1}<\delta}\left(1 \wedge\left\|\mathbf{1}_{\left\{\tau_{N}^{\varepsilon} \geq T\right\}} X_{\mathcal{E}}\left(t_{2}\right)-\mathbf{1}_{\left\{\tau_{N}^{\varepsilon} \geq T\right\}} X_{\mathcal{E}}\left(t_{1}\right)\right\| \mathcal{Z}\right)\right)
\end{aligned}
$$

holds. Note that we used the $\varepsilon$-independent bound (2.29) for the last inequality. To estimate the expectation term in (2.30) we utilize the approximative problem (2.24) directly. We start with the drift term $H_{1, \varepsilon}$. On account of (2.28), $\left(\mathbf{A}_{1}\right)$ and $\left(\mathbf{A}_{2}\right)$ and the BDG inequality, there are a non-decreasing, locally bounded function $a(\cdot) \in$ $C([0,+\infty) ;[0,+\infty))$ and a constant $C>0$ independent of $\varepsilon$ such that we have

$$
\begin{aligned}
& \mathbb{E} \| \int_{t_{1}}^{t_{2}} \mathbf{1}_{\left\{\tau_{N}^{\varepsilon} \geq T\right\}} H_{1, \varepsilon}\left(t^{\prime}, X_{\varepsilon}\left(t^{\prime}\right) \mathrm{d} t^{\prime} \|_{\mathcal{Z}}\right. \\
& \quad \leq\left|t_{2}-t_{1}\right| \mathbb{E} \sup _{t \in\left[0, T \wedge \tau_{N}^{\varepsilon}\right]} \| H_{1, \varepsilon}\left(t, X_{\mathcal{E}}(t) \| \mathcal{Z}\right. \\
& \quad \leq C\left|t_{2}-t_{1}\right| \mathbb{E} \sup _{t \in\left[0, T \wedge \tau_{N}^{\varepsilon}\right]}\left(\chi_{R}^{2}\left(\left\|X_{\varepsilon}\right\|_{\mathcal{V}}\right) k(t) f\left(\left\|X_{\varepsilon}\right\|_{\mathcal{V}}\right)\left\|X_{\varepsilon}\right\|_{\mathcal{X}}+\chi_{R}^{2}\left(\left\|X_{\varepsilon}\right\|_{\mathcal{V}}\right)\left\|g_{\varepsilon}\left(X_{\varepsilon}\right)\right\|_{Z}\right) \\
& \quad \leq C k(T)\left|t_{2}-t_{1}\right| \mathbb{E} \sup _{t \in[0, T]}(f(C N) N+q(N)) \leq C a(N) k(T)\left|t_{2}-t_{1}\right| .
\end{aligned}
$$

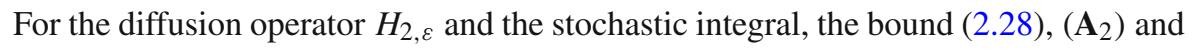
the BDG inequality imply

$$
\begin{aligned}
& \mathbb{E}\left(\| \int_{t_{1}}^{t_{2}} \mathbf{1}_{\left\{\tau_{N}^{\varepsilon} \geq T\right\}} H_{2, \varepsilon}\left(t^{\prime}, X_{\varepsilon}\left(t^{\prime}\right) \mathrm{d} \mathcal{W} \|_{\mathcal{Z}}\right)\right. \\
& \quad \leq \mathbb{E}\left(\sup _{t_{*} \in\left[t_{1}, t_{2}\right]}\left\|\int_{t_{1}}^{t_{*}} \mathbf{1}_{\left\{\tau_{N}^{\varepsilon} \geq T\right\}} H_{2, \varepsilon}\left(t^{\prime}, X_{\varepsilon}\left(t^{\prime}\right)\right) \mathrm{d} \mathcal{W}\right\|_{\mathcal{Z}}\right) \\
& \quad \leq C \mathbb{E}\left(\int_{t_{1}}^{t_{2}} \| \mathbf{1}_{\left\{\tau_{N}^{\varepsilon} \geq T\right\}} H_{2, \varepsilon}\left(t^{\prime}, X_{\varepsilon}\left(t^{\prime}\right) \|_{\mathcal{L}_{2}(\mathbb{U} ; \mathcal{Z})}^{2} \mathrm{~d} \tau\right)^{\frac{1}{2}}\right.
\end{aligned}
$$




$$
\begin{aligned}
& \leq C\left|t_{2}-t_{1}\right|^{\frac{1}{2}} \mathbb{E} \sup _{t \in[0, T]}(q(N) k(t)) \\
& \leq C a(N) k(T)\left|t_{2}-t_{1}\right|^{\frac{1}{2}}
\end{aligned}
$$

Combining the estimates $(2.31),(2.32)$, for any $\delta \in(0,1)$, one has

$$
\begin{aligned}
& \mathbb{E} \sup _{\left[t_{1}, t_{2}\right] \subset\left[0, T \wedge \tau_{N}^{\varepsilon}\right], t_{2}-t_{1}<\delta}\left\|\mathbf{1}_{\left\{\tau_{N}^{\varepsilon} \geq T\right\}} X_{\varepsilon}\left(t_{2}\right)-\mathbf{1}_{\left\{\tau_{N}^{\varepsilon} \geq T\right\}} X_{\varepsilon}\left(t_{1}\right)\right\|_{\mathcal{Z}} \\
& \leq C \mathbb{E} \sup _{\left[t_{1}, t_{2}\right] \subset\left[0, T \wedge \tau_{N}^{\varepsilon}\right], t_{2}-t_{1}<\delta}\left\|\int_{t_{1}}^{t_{2}} \mathbf{1}_{\left\{\tau_{N}^{\varepsilon} \geq T\right\}} H_{1, \varepsilon}\left(t^{\prime}, X_{\varepsilon}\left(t^{\prime}\right)\right) \mathrm{d} t^{\prime}\right\|_{\mathcal{Z}} \\
& \quad+C \mathbb{E} \sup _{\left[t_{1}, t_{2}\right] \subset\left[0, T \wedge \tau_{N}^{\varepsilon}\right], t_{2}-t_{1}<\delta}\left\|\int_{t_{1}}^{t_{2}} \mathbf{1}_{\left\{\tau_{N}^{\varepsilon} \geq T\right\}} H_{2, \varepsilon}\left(t^{\prime}, X_{\mathcal{E}}\left(t^{\prime}\right)\right) \mathrm{d} \mathcal{W}\right\|_{\mathcal{Z}} \\
& \leq \operatorname{Ca}(N) k(T) \delta^{\frac{1}{2}} .
\end{aligned}
$$

Therefore, returning to (2.30), the last estimate implies that for all $\delta \in(0,1)$,

$$
\mathbb{E} \sup _{\left[t_{1}, t_{2}\right] \subset[0, T], t_{2}-t_{1}<\delta}\left(1 \wedge\left\|X_{\varepsilon}\left(t_{2}\right)-X_{\varepsilon}\left(t_{1}\right)\right\|_{\mathcal{Z}}\right) \leq \inf _{N \geq 1}\left\{\frac{C\left(R, X_{0}, T\right)}{N^{2}}+C a(N) k(T) \delta^{\frac{1}{2}}\right\} .
$$

Because $a(\cdot)$ is non-decreasing, we have

$$
\lim _{\delta \rightarrow 0} \sup _{\varepsilon \in(0,1)} \mathbb{E} \sup _{\left[t_{1}, t_{2}\right] \subset[0, T], t_{2}-t_{1}<\delta}\left\|X_{\varepsilon}\left(t_{2}\right)-X_{\varepsilon}\left(t_{1}\right)\right\|_{\mathcal{Z}}=0
$$

Thus, we obtain that, for any $\delta>0$, the limit

$$
\lim _{\delta \rightarrow 0} \sup _{\varepsilon \in(0,1)} \mathbb{P}\left(\sup _{\left[t_{1}, t_{2}\right] \subset[0, T], t_{2}-t_{1}<\delta}\left\|X_{\varepsilon}\left(t_{2}\right)-X_{\varepsilon}\left(t_{1}\right)\right\|_{\mathcal{Z}}>\delta\right)=0
$$

holds. Since $\mathcal{X} \hookrightarrow \hookrightarrow \mathcal{Z}$, for each $t \geq 0, \mathbb{P}\left(X_{\mathcal{E}}(t) \in \cdot\right)$ is tight in $\mathscr{P}(\mathcal{Z})$. This together with (2.34) means for any vanishing sequence $\left\{\varepsilon_{n}\right\}_{n \in \mathbb{N}}$ that (cf. Gawarecki and Mandrekar 2011, Theorem 3.17)

$$
\mu_{\varepsilon_{n}}(\cdot)=\mathbb{P}\left\{X_{\varepsilon_{n}} \in \cdot\right\}
$$

is a tight sequence in $\mathscr{P}(C([0, T] ; \mathcal{Z}))$. On the other hand, since $\mathcal{W}$ stays unchanged, $v_{\varepsilon_{n}}$ defined in (2.25) is also tight.

\subsubsection{Stochastic Compactness}

On the basis of Lemma 2.1 and the weak stochastic compactness theory, we can now characterize the convergence of the sequence $\left\{X_{\varepsilon}\right\}$ obtaining global-in-time results. 
Lemma 2.2 Let $R>1, T>0$. The sequence $\left\{v_{\varepsilon_{n}}\right\}$ defined in Lemma 2.1 has a weakly convergent subsequence, still denoted by $\left\{v_{\varepsilon}\right\}$, with limit measure $v$. There is a probability space $(\widetilde{\Omega}, \widetilde{\mathcal{F}}, \widetilde{\mathbb{P}})$ on which there is a sequence of random variables $\left(\widetilde{X}_{\varepsilon}, \widetilde{\mathcal{W}}_{\varepsilon}\right)$ and a pair $(\widetilde{X}, \widetilde{\mathcal{W}})$ such that we have

$$
\widetilde{\mathbb{P}}\left\{\left(\widetilde{X}_{\varepsilon}, \widetilde{\mathcal{W}}_{\varepsilon}\right) \in \cdot\right\}=v_{n}(\cdot), \quad \widetilde{\mathbb{P}}\{(\widetilde{X}, \tilde{\mathcal{W}}) \in \cdot\}=v(\cdot),
$$

and

$$
\widetilde{X_{\varepsilon}} \rightarrow \widetilde{X} \text { in } C([0, T] ; \mathcal{Z}) \text { and } \widetilde{\mathcal{W}}_{\varepsilon} \rightarrow \widetilde{\mathcal{W}} \text { in } C\left([0, T] ; \mathbb{U}_{0}\right) \widetilde{\mathbb{P}}-\text { a.s. }
$$

Moreover, for $t \in[0, T]$, the following results hold.

(i) $\widetilde{\mathcal{W}}_{\varepsilon}$ is a cylindrical Wiener process with respect to $\widetilde{\mathcal{F}}_{t}^{\varepsilon}=\sigma\left\{\widetilde{X}_{\varepsilon}(\tau), \widetilde{\mathcal{W}}_{\varepsilon}(\tau)\right\}_{\tau \in[0, t]}$.

(ii) $\tilde{\mathcal{W}}$ is a cylindrical Wiener process with respect to $\widetilde{\mathcal{F}}_{t}=\sigma\{\tilde{X}(\tau), \tilde{\mathcal{W}}(\tau)\}_{\tau \in[0, t]^{\circ}}$. (iii) On $\left(\widetilde{\Omega}, \widetilde{\mathcal{F}}, \widetilde{\mathbb{P}},\left\{\widetilde{\mathcal{F}}_{t}^{\varepsilon}\right\}_{t \geq 0}\right)$, we have that $\widetilde{\mathbb{P}}-$ a.s.

$$
\begin{aligned}
\widetilde{X}_{\varepsilon}(t)-\widetilde{X_{\varepsilon}}(0)= & \int_{0}^{t} \chi_{R}^{2}\left(\left\|\widetilde{X_{\varepsilon}}\right\| \mathcal{V}\right)\left[b\left(t^{\prime}, \widetilde{X_{\varepsilon}}\right)+g_{\varepsilon}\left(t^{\prime}, \widetilde{X}_{\varepsilon}\right)\right] \mathrm{d} t^{\prime} \\
& +\int_{0}^{t} \chi_{R}\left(\left\|\widetilde{X_{\varepsilon}}\right\| \mathcal{V}\right) h_{\varepsilon}\left(t^{\prime}, \widetilde{X}_{\varepsilon}\right) \mathrm{d} \widetilde{\mathcal{W}}_{\varepsilon} .
\end{aligned}
$$

Proof The existence of the sequence $\left(\widetilde{X}_{\varepsilon}, \widetilde{\mathcal{W}}_{\varepsilon}\right)$ satisfying $(2.36)$ is a consequence of Lemma 2.1 and Theorems A.6 and A.7 . Besides, Breit et al. (2018, Theorem 2.1.35 and Corollary 2.1.36) imply that $\widetilde{\mathcal{W}}_{\varepsilon}$ and $\widetilde{\mathcal{W}}$ are cylindrical Wiener processes relative to $\widetilde{\mathcal{F}}_{t}^{\varepsilon}=\sigma\left\{\widetilde{X}_{\varepsilon}(\tau), \widetilde{\mathcal{W}}_{\varepsilon}(\tau)\right\}_{\tau \in[0, t]}$ and $\widetilde{\mathcal{F}}_{t}=\sigma\{\widetilde{X}(\tau), \widetilde{\mathcal{W}}(\tau)\}_{\tau \in[0, t]}$, respectively. As in Bensoussan (1995, (4.17) \& page 282) or Breit et al. (2018, Theorem 2.9.1), one can find that $\left(\widetilde{X}_{\varepsilon}, \widetilde{\mathcal{W}}_{\varepsilon}\right)$ relative to $\left\{\widetilde{\mathcal{F}}_{t}^{\varepsilon}\right\}_{t \geq 0}$ satisfies $(2.37) \widetilde{\mathbb{P}}-$ a.s.

\subsubsection{Concluding the Proof of (i) in Theorem 2.1}

To begin with, we notice that the embedding $\mathcal{X} \hookrightarrow \mathcal{Z}$ is continuous, which means there exist continuous maps $\pi_{m}: \mathcal{Z} \rightarrow \mathcal{X}, m \geq 1$ such that

$$
\left\|\pi_{m} x\right\|_{\mathcal{X}} \leq\|x\|_{\mathcal{X}}, \quad \lim _{m \rightarrow \infty}\left\|\pi_{m} x\right\|_{\mathcal{X}}=\|x\|_{\mathcal{X}}, \quad x \in \mathcal{Z},
$$

where $\|x\|_{\mathcal{X}}:=\infty$ if $x \notin \mathcal{X}$. This, together with (2.27), (2.35) and Fatou's lemma, yields

$$
\begin{aligned}
\widetilde{\mathbb{E}} \sup _{t \in[0, T]}\|\widetilde{X}\|_{\mathcal{X}}^{2} & \leq \liminf _{m \rightarrow \infty} \widetilde{\mathbb{E}} \sup _{t \in[0, T]}\left\|\pi_{m} \widetilde{X}\right\|_{\mathcal{X}}^{2} \\
& \leq \liminf _{m \rightarrow \infty} \liminf _{\varepsilon \rightarrow 0} \widetilde{\mathbb{E}} \sup _{t \in[0, T]}\left\|\pi_{m} \widetilde{X}_{\mathcal{E}}\right\|_{\mathcal{X}}^{2} \\
& \leq \liminf _{m \rightarrow \infty} \liminf _{\varepsilon \rightarrow 0} \mathbb{E} \sup _{t \in[0, T]}\left\|X_{\mathcal{\varepsilon}}\right\|_{\mathcal{X}}^{2}<C\left(R, X_{0}, T\right) .
\end{aligned}
$$


Using (2.36), (2.38), $\mathcal{X} \hookrightarrow \mathcal{V},\left(\mathbf{A}_{2}\right)$ and Lemma A.8 (up to further subsequence) in (2.37), we obtain that

$$
\int_{0}^{t} \chi_{R}\left(\left\|\widetilde{X_{\varepsilon}}\right\|_{\mathcal{V}}\right) h_{\varepsilon}\left(t, \widetilde{X_{\varepsilon}}\right) \mathrm{d} \widetilde{\mathcal{W}_{\varepsilon}} \stackrel{\varepsilon \rightarrow 0}{\longrightarrow} \int_{0}^{t} \chi_{R}\left(\|\widetilde{X}\|_{\mathcal{V}}\right) h(t, \widetilde{X}) \mathrm{d} \widetilde{\mathcal{W}} \text { in } L^{2}(0, T ; \mathcal{Z}) \mathbb{P}-\text { a.s. }
$$

As before, it follows from (2.36), (2.38), $\mathcal{X} \hookrightarrow \mathcal{V}$ and $\left(\mathbf{A}_{2}\right)$ that for any $t \in[0, T]$ and $\phi \in \mathcal{Z}^{*}$,

$$
\begin{aligned}
& \int_{0}^{t} \chi_{R}^{2}(\|\widetilde{X}\| \mathcal{V})_{\mathcal{Z}}\left\langle b\left(s, \widetilde{X}_{\varepsilon}(s)\right)-b(s, \widetilde{X}(s))\right. \\
& \left.\quad+g_{\varepsilon}\left(s, \widetilde{X}_{\varepsilon}(s)\right)-g(s, \widetilde{X}(s)), \phi\right\rangle \mathcal{Z}^{*} \mathrm{~d} s \stackrel{\varepsilon \rightarrow 0}{\longrightarrow} 0 \mathbb{P}-\text { a.s. }
\end{aligned}
$$

Therefore, we derive that for all $\phi \in \mathcal{Z}^{*}$ and $\mathrm{d} t \otimes \widetilde{\mathbb{P}}-$ a.s.,

$$
\begin{aligned}
& \mathcal{Z}\langle\tilde{X}(t), \phi\rangle_{\mathcal{Z}^{*}-\mathcal{Z}}\langle\tilde{X}(0), \phi\rangle_{\mathcal{Z}^{*}} \\
& =\int_{0}^{t} \chi_{R}^{2}\left(\|\widetilde{X}\|_{\mathcal{V}}\right)_{\mathcal{Z}}\left\langle b(s, \widetilde{X}(s))+g\left(s, \widetilde{X}_{\varepsilon}(s)\right), \phi\right\rangle_{\mathcal{Z}^{*}} \mathrm{~d} s \\
& \quad+\mathcal{Z}\left\langle\int_{0}^{t} \chi_{R}\left(\|\tilde{X}\|_{\mathcal{V}}\right) h(t, \tilde{X}) \mathrm{d} \tilde{\mathcal{W}}, \phi\right\rangle_{\mathcal{Z}^{*}}
\end{aligned}
$$

Due to $(2.38),\left(\mathbf{A}_{1}\right)$ and $\left(\mathbf{A}_{2}\right)$, we see that $t \mapsto \int_{0}^{t} \chi_{R}\left(\|\tilde{X}\|_{\mathcal{V}}\right) h\left(t^{\prime}, \tilde{X}\left(t^{\prime}\right)\right) \mathrm{d} \tilde{\mathcal{W}}$ is a local continuous martingale on $\mathcal{Y} \subset \mathcal{Z}$, and that $t \mapsto \int_{0}^{t} \chi_{R}^{2}(\|\widetilde{X}\| \mathcal{V})\left[b\left(t^{\prime}\right.\right.$, $\left.\left.\tilde{X}\left(t^{\prime}\right)\right)+g\left(t^{\prime}, \tilde{X}\left(t^{\prime}\right)\right)\right] \mathrm{d} t^{\prime}$ is a continuous process on $\mathcal{Z}$ as well. Hence, we obtain that $\tilde{X}$ is a global martingale solution to (2.23). Moreover, (2.36) and (2.38) imply that $\widetilde{X} \in L^{2}\left(\widetilde{\Omega} ; L^{\infty}(0, T ; \mathcal{X}) \cap C([0, T] ; \mathcal{Z})\right)$ holds. Define

$$
\tilde{\tau}=\inf \{t \geq 0:\|\widetilde{X}(t)\| \mathcal{V}>R\}
$$

then we see that $(\widetilde{\mathcal{S}}, \tilde{X}, \tilde{\tau})$ is a local martingale solution to (1.1), where $\widetilde{\mathcal{S}}=$ $\left(\widetilde{\Omega}, \widetilde{\mathcal{F}}, \widetilde{\mathbb{P}},\left\{\widetilde{\mathcal{F}}_{t}\right\}_{t \geq 0}, \widetilde{\mathcal{W}}\right)$ with $\left\{\widetilde{\mathcal{F}}_{t}\right\}_{t \geq 0}=\sigma\{\tilde{X}(\tau), \tilde{\mathcal{W}}(\tau)\}_{\tau \in[0, t]}$. We have finished the proof.

\subsection{Proof of (ii) in Theorem 2.1}

To obtain a pathwise solution to (1.1), we will use (i) in Theorem 2.1 and the GyöngyKrylov Lemma, cf. Lemma A.9. The proof can naturally be broken down into several subsections.

\subsubsection{Pathwise Uniqueness}

We first state the following result which indicates that for $L^{\infty}(\Omega)$-initial values, the solution map is time locally Lipschitz in the less regular space $\mathcal{Z}$. 
Lemma 2.3 Let $\mathcal{S}=\left(\Omega, \mathcal{F}, \mathbb{P},\left\{\mathcal{F}_{t}\right\}_{t \geq 0}, \mathcal{W}\right)$ be a fixed stochastic basis and let $\left(\boldsymbol{B}_{1}\right)$ hold. Let $M>0$ be a constant. Assume that $X_{0}$ and $Y_{0}$ are two $\mathcal{X}$-valued $\mathcal{F}_{0^{-}}$ measurable random variables satisfying $\left\|X_{0}\right\|_{\mathcal{X}},\left\|Y_{0}\right\|_{\mathcal{X}}<M$ almost surely.

Let $\left(\mathcal{S}, X, \tau_{1}\right)$ and $\left(\mathcal{S}, Y, \tau_{2}\right)$ be two local pathwise solutions to $(1.1)$ such that $X(0)=$ $X_{0}, Y(0)=Y_{0}$ almost surely, and $X\left(\cdot \wedge \tau_{1}\right), Y\left(\cdot \wedge \tau_{2}\right) \in L^{2}(\Omega ; C([0, \infty) ; \mathcal{X}))$ for $i=1,2$.

Then, for any $T>0$, there exists a constant $C(M, T)>0$ such that

$$
\mathbb{E} \sup _{t \in\left[0, \tau_{X, Y}^{T}\right]}\|X(t)-Y(t)\|_{\mathcal{Z}}^{2} \leq C(M, T) \mathbb{E}\left\|X_{0}-Y_{0}\right\|_{\mathcal{Z}}^{2}
$$

In (2.39) we used

$$
\tau_{X}^{T}:=\inf \left\{t \geq 0:\|X(t)\|_{\mathcal{X}}>M+2\right\} \wedge T, \quad \tau_{Y}^{T}:=\inf \left\{t \geq 0:\|Y(t)\|_{\mathcal{X}}>M+2\right\} \wedge T,
$$

and $\tau_{X, Y}^{T}:=\tau_{X}^{T} \wedge \tau_{Y}^{T}$

Proof Let $Z=X-Y$. Then, $Z$ satisfies the following equation

$$
\begin{aligned}
\mathrm{d}\|Z\|_{\mathcal{Z}}^{2}=2( & {[h(t, X)-h(t, Y)] \mathrm{d} \mathcal{W}, Z)_{\mathcal{Z}}+2(b(t, X)-b(t, Y), Z)_{\mathcal{Z}} \mathrm{d} t } \\
& +2(g(t, X)-g(t, Y), Z)_{\mathcal{Z}} \mathrm{d} t+\|h(t, X)-h(t, X)\|_{\mathcal{L}_{2}(\mathbb{U} ; \mathcal{Z})}^{2} \mathrm{~d} t
\end{aligned}
$$

By $\left(\mathbf{A}_{1}\right),\left(\mathbf{B}_{1}\right)$, Itô's formula (which holds true on the entire space $\mathcal{Z}$ ), and the BDG inequality, we find for some $C>0$ depending on $b, g, h$ the estimate

$$
\begin{aligned}
\mathbb{E} & \sup _{t \in\left[0, \tau_{X, Y}^{T}\right]}\|Z(t)\|_{\mathcal{Z}}^{2}-\mathbb{E}\|Z(0)\|_{\mathcal{Z}}^{2} \\
\leq & C \mathbb{E}\left(\int_{0}^{\tau_{X, Y}^{T}}\|h(t, X)-h(t, Y)\|_{\mathcal{L}_{2}(\mathbb{U}, \mathcal{Z})}^{2}\|Z\|_{\mathcal{Z}}^{2} \mathrm{~d} t\right)^{\frac{1}{2}}+\mathbb{E} \int_{0}^{\tau_{X, Y}^{T}} q(M+2) k(t)\|Z(t)\|_{\mathcal{Z}}^{2} \mathrm{~d} t \\
\leq & C q(M+2) \mathbb{E}\left(\sup _{t \in\left[0, \tau_{X, Y}^{T}\right]}\|Z\|_{\mathcal{Z}}^{2} \cdot \int_{0}^{\tau_{X, Y}^{T}} k^{2}(t)\|Z\|_{\mathcal{Z}}^{2} \mathrm{~d} t\right)^{\frac{1}{2}} \\
& +C q(M+2) \int_{0}^{T} k(t) \mathbb{E} \sup _{t^{\prime} \in\left[0, \tau_{X, Y}^{t}\right]}\left\|Z\left(t^{\prime}\right)\right\|_{\mathcal{Z}}^{2} \mathrm{~d} t \\
\leq & \frac{1}{2} \mathbb{E} \sup _{t \in\left[0, \tau_{X, Y}^{T}\right]}\|Z\|_{\mathcal{Z}}^{2}+C(M, T) \int_{0}^{T} \operatorname{Es}_{t^{\prime} \in\left[0, \tau_{X, Y}^{t}\right]}\left\|Z\left(t^{\prime}\right)\right\|_{\mathcal{Z}}^{2} \mathrm{~d} t .
\end{aligned}
$$

If we apply Grönwall's inequality to the estimate above, we get (2.39).

Lemma 2.4 Let $\mathcal{S}=\left(\Omega, \mathcal{F}, \mathbb{P},\left\{\mathcal{F}_{t}\right\}_{t \geq 0}, \mathcal{W}\right)$ be a fixed stochastic basis and let $\left(\boldsymbol{B}_{1}\right)$ hold. Let $X_{0}$ be an $\mathcal{X}$-valued $\mathcal{F}_{0}$-measurable random variable satisfying $\mathbb{E}\left\|X_{0}\right\|_{\mathcal{X}}^{2}<$ $\infty$. If $\left(\mathcal{S}, X_{1}, \tau_{1}\right)$ and $\left(\mathcal{S}, X_{2}, \tau_{2}\right)$ are two local pathwise solutions to (1.1) satisfying 
$X_{i}\left(\cdot \wedge \tau_{i}\right) \in L^{2}(\Omega ; C([0, \infty) ; \mathcal{X}))$ for $i=1,2$ and $\mathbb{P}\left\{X_{1}(0)=X_{2}(0)=X_{0}\right\}=1$, then

$$
\mathbb{P}\left\{X_{1}=X_{2}, \forall t \in\left[0, \tau_{1} \wedge \tau_{2}\right]\right\}=1
$$

Proof We first assume that $\left\|X_{0}\right\|_{\mathcal{X}}<M \mathbb{P}-$ a.s. for some deterministic $M>0$. For any $K>2 M$ and $T>0$, we define

$$
\tau_{K}^{T}:=\inf \left\{t \geq 0:\left\|X_{1}(t)\right\|_{\mathcal{X}}+\left\|X_{2}(t)\right\|_{\mathcal{X}}>K\right\} \wedge T .
$$

Then, one can repeat all steps in the proof of (2.39) by using $\tau_{K}^{T}$ instead of $\tau_{X, Y}^{T}$ to find

$$
\mathbb{E} \sup _{t \in\left[0, \tau_{K}^{T}\right]}\left\|X_{1}(t)-X_{2}(t)\right\|_{\mathcal{Z}}^{2} \leq C(K, T) \mathbb{E}\left\|X_{1}(0)-X_{2}(0)\right\|_{\mathcal{Z}}^{2}=0
$$

It is easy to see that

$$
\mathbb{P}\left\{\liminf _{K \rightarrow \infty} \tau_{K}^{T} \geq \tau_{1} \wedge \tau_{2} \wedge T\right\}=1
$$

Sending $K \rightarrow \infty$, using the monotone convergence theorem and (2.41) with noticing $T>0$ is arbitrary, we obtain the desired result for $X_{0}$ being almost surely bounded.

It remains to remove this restriction. Motivated by Glatt-Holtz and Ziane (2009) and Glatt-Holtz and Vicol (2014), for general $\mathcal{X}$-valued $\mathcal{F}_{0}$-measurable initial data such that $\mathbb{E}\left\|X_{0}\right\|_{\mathcal{X}}^{2}<\infty$ holds, we define $\Omega_{k}=\left\{k-1 \leq\left\|X_{0}\right\|_{\mathcal{X}}<k\right\}, k \geq 1$. Then, we see that $\Omega_{k} \bigcap \Omega_{k^{\prime}}=\emptyset$ for $k \neq k^{\prime}$, and $\bigcup_{k \geq 1} \Omega_{k}$ is a set of full measure. Consider

$$
X_{0}(\omega)=\sum_{k \geq 1} X_{0}(\omega, x) \mathbf{1}_{\Omega_{k}}:=\sum_{k \geq 1} X_{0, k}(\omega) \mathbb{P}-\text { a.s. }
$$

Notice that

$$
\begin{aligned}
& \mathbf{1}_{\Omega_{k}} X_{1}\left(t \wedge \tau_{1}\right)-\mathbf{1}_{\Omega_{k}} X(0) \\
& =\mathbf{1}_{\Omega_{k}} \int_{0}^{t \wedge \tau_{1}} b\left(t^{\prime}, X_{1}\right) \mathrm{d} t^{\prime}+\mathbf{1}_{\Omega_{k}} \int_{0}^{t \wedge \tau_{1}} g\left(t^{\prime}, X_{1}\right) \mathrm{d} t^{\prime}+\mathbf{1}_{\Omega_{k}} \int_{0}^{t \wedge \tau_{1}} h\left(t, X_{1}\right) \mathrm{d} \mathcal{W} \\
& =\int_{0}^{t \wedge \mathbf{1}_{\Omega_{k}} \tau_{1}} \mathbf{1}_{\Omega_{k}} b\left(t^{\prime}, X_{1}\right) \mathrm{d} t^{\prime}+\int_{0}^{t \wedge \mathbf{1}_{\Omega_{k}} \tau_{1}} \mathbf{1}_{\Omega_{k}} g\left(t^{\prime}, X_{1}\right) \mathrm{d} t^{\prime}+\int_{0}^{t \wedge \mathbf{1}_{\Omega_{k}} \tau_{1}} \mathbf{1}_{\Omega_{k}} h\left(t^{\prime}, X_{1}\right) \mathrm{d} \mathcal{W} .
\end{aligned}
$$

Notice that $\mathbf{1}_{\Omega_{k}} F\left(t, X_{1}\right)=F\left(t, \mathbf{1}_{\Omega_{k}} X_{1}\right)-\mathbf{1}_{\Omega_{k}^{C}} F(t, \mathbf{0})$ for $F \in\{b, g, h\}$, and $\left(\mathbf{A}_{1}\right),\left(\mathbf{A}_{2}\right)$ implies $\|b(t, \mathbf{0})\| \mathcal{X},\|g(t, \mathbf{0})\|_{\mathcal{Z}},\|h(t, \mathbf{0})\|_{\mathcal{L}_{2}(\mathbb{U} ; \mathcal{Y})}<\infty$. Then, we can proceed with 


$$
\begin{aligned}
\mathbf{1}_{\Omega_{k}} X_{1}\left(t \wedge \mathbf{1}_{\Omega_{k}} \tau_{1}\right)-X_{0, k} \\
=\int_{0}^{t \wedge \mathbf{1}_{\Omega_{k}} \tau_{1}} b\left(t^{\prime}, \mathbf{1}_{\Omega_{k}} X_{1}\right) \mathrm{d} t^{\prime} \\
\quad+\int_{0}^{t \wedge \mathbf{1}_{\Omega_{k}} \tau_{1}} g\left(t^{\prime}, \mathbf{1}_{\Omega_{k}} X_{1}\right) \mathrm{d} t^{\prime}+\int_{0}^{t \wedge \mathbf{1}_{\Omega_{k}} \tau_{1}} h\left(t^{\prime}, \mathbf{1}_{\Omega_{k}} X_{1}\right) \mathrm{d} \mathcal{W} \mathbb{P}-\text { a.s. },
\end{aligned}
$$

which means that $\left(\mathbf{1}_{\Omega_{k}} X_{1}, \mathbf{1}_{\Omega_{k}} \tau_{1}\right)$ is a solution to (1.1) with initial data $X_{0, k}$.

Similarly, $\left(\mathbf{1}_{\Omega_{k}} X_{2}, \mathbf{1}_{\Omega_{k}} \tau_{2}\right)$ is also a solution to (1.1) with initial data $X_{0, k}$. Altogether we obtain $\mathbf{1}_{\Omega_{k}} X_{1}=\mathbf{1}_{\Omega_{k}} X_{2}$ on $\left[0, \mathbf{1}_{\Omega_{k}} \tau_{1} \wedge \mathbf{1}_{\Omega_{k}} \tau_{2}\right]$ almost surely. Because $X_{i}=\sum_{k \geq 1} X_{i} \mathbf{1}_{\Omega_{k}}$ and $\tau_{i}=\sum_{k \geq 1} \tau_{i} \mathbf{1}_{\Omega_{k}}$ almost surely for $i=1,2, \Omega_{k} \bigcap \Omega_{k^{\prime}}=\emptyset$ for $k \neq k^{\prime}$ and $\bigcup_{k \geq 1} \Omega_{k}$ is a set of full measure, we have

$$
\mathbb{P}\left\{X_{1}=X_{2} \forall t \in\left[0, \tau_{1} \wedge \tau_{2}\right]\right\} \geq \mathbb{P}\left\{\cup_{k \geq 1} \Omega_{k}\right\}=1,
$$

which completes the proof.

For the cut-off problem (2.23), we also have pathwise uniqueness. Indeed, since $\mathcal{Z} \hookrightarrow$ $\mathcal{V}$, the additional terms coming from the cut-off function $\chi_{R}(\cdot)$ can be handled by the mean value theorem as

$$
\left|\chi_{R}\left(\left\|X_{1}\right\| \mathcal{V}\right)-\chi_{R}\left(\left\|X_{2}\right\| \mathcal{V}\right)\right| \leq C\left\|X_{1}-X_{2}\right\| \mathcal{V} \leq C\left\|X_{1}-X_{2}\right\|_{\mathcal{Z}}
$$

Then, one can modify the proof of Lemma 2.4 in a straightforward way to get

Lemma 2.5 Let $T>0$ and $\mathcal{S}=\left(\Omega, \mathcal{F}, \mathbb{P},\left\{\mathcal{F}_{t}\right\}_{t \geq 0}, \mathcal{W}\right)$ be a fixed stochastic basis. Let $\left(\boldsymbol{B}_{1}\right)$ hold and let $X_{0}$ be an $\mathcal{X}$-valued $\mathcal{F}_{0}$-measurable random variable satisfying $\mathbb{E}\left\|X_{0}\right\|_{\mathcal{X}}^{2}<\infty$.

If $\left(\mathcal{S}, X_{1}, T\right)$ and $\left(\mathcal{S}, X_{2}, T\right)$ are two solutions, on the same basis $\mathcal{S}$, of (2.23) such that $\mathbb{P}\left\{X_{1}(0)=X_{2}(0)=X_{0}\right\}=1$ and $X_{i} \in L^{2}(\Omega ; C([0, T) ; \mathcal{X}))$ for $i=1,2$, then

$$
\mathbb{P}\left\{X_{1}=X_{2} \forall t \in[0, T]\right\}=1 \text {. }
$$

\subsubsection{Pathwise Solution to the Cut-Off Problem}

Now we prove the existence and uniqueness of a pathwise solution to (2.23). To be more precise, we are going to show the following result.

Lemma 2.6 Let $\mathcal{S}=\left(\Omega, \mathcal{F}, \mathbb{P},\left\{\mathcal{F}_{t}\right\}_{t \geq 0}, \mathcal{W}\right)$ be a fixed stochastic basis. Let $X_{0} \in$ $L^{2}(\Omega ; \mathcal{X})$ be an $\mathcal{F}_{0}$-measurable random variable.

If Assumptions (A)-(B) hold, then (2.23) has a unique global pathwise solution $X$ which satisfies for any $T>0$

$$
X \in L^{2}(\Omega ; C([0, T] ; \mathcal{X})) .
$$


Proof Uniqueness is a direct consequence of Lemma 2.5. The proof of the other assertions is divided into two steps.

Step 1: Existence Let $\mathcal{S}=\left(\Omega, \mathcal{F}, \mathbb{P},\left\{\mathcal{F}_{t}\right\}_{t \geq 0}, \mathcal{W}\right)$ be given and let $X_{\varepsilon}$ be the global pathwise solution to (2.24). We define sequences of measures $v_{\mathcal{E}^{(1)}, \varepsilon^{(2)}}$ and $\mu_{\varepsilon^{(1)}, \varepsilon^{(2)}}$ as

$$
v_{\varepsilon^{(1)}, \varepsilon^{(2)}}(\cdot)=\mathbb{P}\left\{\left(X_{\varepsilon^{(1)}}, X_{\varepsilon^{(2)}}\right) \in \cdot\right\} \text { on } C([0, T] ; \mathcal{Z}) \times C([0, T] ; \mathcal{Z})
$$

and

$$
\begin{aligned}
\mu_{\varepsilon^{(1)}, \varepsilon^{(2)}}(\cdot)= & \mathbb{P}\left\{\left(X_{\mathcal{E}^{(1)}}, X_{\varepsilon^{(2)}}, \mathcal{W}\right) \in \cdot\right\} \text { on } C([0, T] ; \mathcal{Z}) \times C([0, T] ; \mathcal{Z}) \\
& \times C\left([0, T] ; \mathbb{U}_{0}\right) .
\end{aligned}
$$

Let $\left\{v_{\varepsilon_{k}^{(1)}, \varepsilon_{k}^{(2)}}\right\}_{k \in \mathbb{N}}$ be an arbitrary subsequence of $\left\{v_{\varepsilon^{(1)}, \varepsilon^{(2)}}\right\}_{n \in \mathbb{N}}$ such that $\varepsilon_{k}^{(1)}, \varepsilon_{k}^{(2)} \rightarrow$ 0 as $k \rightarrow \infty$. With minor modifications in the proof of Lemma 2.1, the tightness of $\left\{v_{\varepsilon_{k}}^{(1)}, \varepsilon_{k}^{(2)}\right\}_{k \in \mathbb{N}}$ can be obtained. Similar to Lemma 2.2, one can find a probability space $(\widetilde{\Omega}, \widetilde{\mathcal{F}}, \widetilde{\mathbb{P}})$ on which there is a sequence of random variables $(\underbrace{}_{\varepsilon_{k}^{(1)}}, \overline{X_{\varepsilon_{k}^{(2)}}}, \widetilde{\mathcal{W}_{k}})$ and a random variable $(\underline{X}, \bar{X}, \tilde{\mathcal{W}})$ such that

$$
\begin{aligned}
& (\underbrace{}_{\varepsilon_{k}^{(1)}}, \overline{X_{\varepsilon_{k}^{(2)}}}, \widetilde{\mathcal{W}_{k}}) \underset{k \rightarrow \infty}{\longrightarrow}(\underline{X}, \bar{X}, \widetilde{\mathcal{W}}) \text { in } C([0, T] ; \mathcal{Z}) \\
& \quad \times C([0, T] ; \mathcal{Z}) \times C\left([0, T] ; \mathbb{U}_{0}\right) \widetilde{\mathbb{P}}-\text { a.s. }
\end{aligned}
$$

Then, $v_{\mathcal{E}^{(1)}, \varepsilon^{(2)}}$ converges weakly to a measure $v$ on $C([0, T] ; \mathcal{Z}) \times C([0, T] ; \mathcal{Z})$ defined by

$$
v(\cdot)=\widetilde{\mathbb{P}}\{(\underline{X}, \bar{X}) \in \cdot\}
$$

Going along the lines as in Sect. 2.3.3, we see that both $(\widetilde{\mathcal{S}}, \underline{X}, T)$ and $(\widetilde{\mathcal{S}}, \bar{X}, T)$ are martingale solutions to (2.23) such that $\underline{X}, \bar{X} \in L^{2}\left(\widetilde{\Omega} ; L^{\infty}(0, T ; \mathcal{X}) \cap C([0, T] ; \mathcal{Z})\right)$. Moreover, since $X_{\varepsilon}(0) \equiv X_{0}$ for all $n$, we have that $\underline{X}(0)=\bar{X}(0)$ almost surely in $\widetilde{\Omega}$. Then, we use Lemma 2.5 to see

$$
v(\{(\underline{X}, \bar{X}) \in C([0, T] ; \mathcal{Z}) \times C([0, T] ; \mathcal{Z}), \underline{X}=\bar{X}\})=1 .
$$

Lemma A.9 implies that the original sequence $\left\{X_{\varepsilon}\right\}$ defined on the initial probability space $(\Omega, \mathcal{F}, \mathbb{P})$ has a subsequence (still labeled in the same way) satisfying

$$
X_{\varepsilon} \rightarrow X \text { in } C([0, T] ; \mathcal{Z})
$$

for some $X$ in $C([0, T] ; \mathcal{Z})$. Similar to $(2.38)$, we have 


$$
\begin{aligned}
\mathbb{E} \sup _{t \in[0, T]}\|X\|_{\mathcal{X}}^{2} & \leq \liminf _{m \rightarrow \infty} \mathbb{E} \sup _{t \in[0, T]}\left\|\pi_{m} X\right\|_{\mathcal{X}}^{2} \\
& \leq \liminf _{m \rightarrow \infty} \liminf _{\varepsilon \rightarrow 0} \mathbb{E} \sup _{t \in[0, T]}\left\|\pi_{m} X_{\mathcal{E}}\right\|_{\mathcal{X}}^{2} \\
& \leq \liminf _{m \rightarrow \infty} \liminf _{\varepsilon \rightarrow 0} \mathbb{E} \sup _{t \in[0, T]}\left\|X_{\mathcal{E}}\right\|_{\mathcal{X}}^{2}<C\left(R, X_{0}, T\right) .
\end{aligned}
$$

Therefore, $X \in L^{2}\left(\Omega ; L^{\infty}(0, T ; \mathcal{X}) \cap C([0, T] ; \mathcal{Z})\right)$. Since for each $n, X_{\varepsilon}$ is $\left\{\mathcal{F}_{t}\right\}_{t \geq 0}$ progressive measurable, so is $X$. Using (2.44) and the embedding $\mathcal{Z} \hookrightarrow \mathcal{V}$, we obtain a global pathwise solution to (2.23).

Step 2: Time continuity As $X \in L^{2}\left(\Omega ; L^{\infty}(0, T ; \mathcal{X}) \cap C([0, T] ; \mathcal{Z})\right)$, now we only need to prove that $X(t)$ is continuous in $\mathcal{X}$. Since $\mathcal{X} \hookrightarrow \mathcal{Z}$ is dense, we see that $X$ is weakly continuous in $\mathcal{X}$ (cf. Temam 1977, page 263, Lemma 1.4). It suffices to prove the continuity of $[0, T] \ni t \mapsto\|X(t)\| \mathcal{X}$. The difficulty here is that the problem (1.1) is singular, i.e., $g(t, X)$ is only a $\mathcal{Z}$-valued process and $h(t, X)$ is only an $\mathcal{L}_{2}(\mathbb{U} ; \mathcal{Y})$-valued process; hence, the products $(g(t, X), X) \mathcal{X}$ and $\left(h(t, X) e_{i}, X\right) \mathcal{X}$ might not exist and the classical Itô formula in the Hilbert space $\mathcal{X}$ (see Da Prato and Zabczyk 2014, Theorem 4.32 or Gawarecki and Mandrekar 2011, Theorem 2.10) cannot be used directly here. At this point the regularization operator $T_{\varepsilon}$ from $\left(\mathbf{B}_{2}\right)$ is invoked to consider the Itô formula for $\left\|T_{\varepsilon} X\right\|_{\mathcal{X}}^{2}$ instead. Then, we have

$$
\begin{aligned}
\mathrm{d}\left\|T_{\mathcal{E}} X\right\|_{\mathcal{X}}^{2}= & 2 \chi_{R}\left(\|X\|_{\mathcal{V}}\right)\left(T_{\varepsilon} h(t, X) \mathrm{d} \mathcal{W}, T_{\varepsilon} X\right)_{\mathcal{X}}+2 \chi_{R}^{2}\left(\|X\|_{\mathcal{V}}\right)\left(T_{\varepsilon} b(t, X), T_{\varepsilon} X\right)_{\mathcal{X}} \mathrm{d} t \\
& +2 \chi_{R}^{2}\left(\|X\|_{\mathcal{V}}\right)\left(T_{\varepsilon} g(t, X), T_{\varepsilon} X\right)_{\mathcal{X}} \mathrm{d} t+\chi_{R}^{2}\left(\|X\|_{\mathcal{V}}\right)\left\|T_{\varepsilon} h(t, X)\right\|_{\mathcal{L}_{2}(\mathbb{U} ; \mathcal{X})}^{2} \mathrm{~d} t
\end{aligned}
$$

By (2.45),

$$
\tau_{N}=\inf \left\{t \geq 0:\|X\|_{\mathcal{X}}>N\right\} \rightarrow \infty \text { as } N \rightarrow \infty \mathbb{P}-\text { a.s. }
$$

Thus, we only need to prove the continuity up to time $\tau_{N} \wedge T$ for each $N \geq 1$. Using $\left(\mathbf{B}_{2}\right),\left(\mathbf{A}_{1}\right)$ and the bound $\chi_{R}\left(\|X\|_{\mathcal{V}}\right) \leq 1$, we have for $\left[t_{2}, t_{1}\right] \subset[0, T]$ with $t_{1}-t_{2}<1$ the estimate

$$
\mathbb{E}\left[\left(\left\|T_{\varepsilon} X\left(t_{1} \wedge \tau_{N}\right)\right\|_{\mathcal{X}}^{2}-\left\|T_{\varepsilon} X\left(t_{2} \wedge \tau_{N}\right)\right\|_{\mathcal{X}}^{2}\right)^{4}\right] \leq C(N, T)\left|t_{1}-t_{2}\right|^{2}
$$

Using Fatou's lemma, we arrive at

$$
\mathbb{E}\left[\left(\left\|X\left(t_{1} \wedge \tau_{N}\right)\right\|_{\mathcal{X}}^{2}-\left\|X\left(t_{2} \wedge \tau_{N}\right)\right\|_{\mathcal{X}}^{2}\right)^{4}\right] \leq C(N, T)\left|t_{1}-t_{2}\right|^{2},
$$

which together with Kolmogorov's continuity theorem ensures the continuity of $t \mapsto$ $\left\|X\left(t \wedge \tau_{N}\right)\right\| \mathcal{X}$.

With Lemma 2.6 at hand, we are in the position to finish the proof of (ii) in Theorem 2.1. 


\subsubsection{Concluding the Proof of (ii) in Theorem 2.1}

Similar to Lemma 2.4 , for $X_{0}(\omega, x) \in L^{2}(\Omega ; \mathcal{X})$, we let

$$
\Omega_{k}=\left\{k-1 \leq\left\|X_{0}\right\| \mathcal{X}<k\right\}, \quad k \geq 1,
$$

and recall (2.42). On account of Lemma 2.6, we let $X_{k, R}$ be the global pathwise solution to the cut-off problem (2.23) with initial value $X_{0, k}$ and cut-off function $\chi_{R}(\cdot)$. Define

$$
\tau_{k, R}=\inf \left\{t>0: \sup _{t^{\prime} \in[0, t]}\left\|X_{k, R}\left(t^{\prime}\right)\right\|_{\mathcal{X}}^{2}>\left\|X_{0, k}\right\|_{\mathcal{X}}^{2}+2\right\} .
$$

Since $X_{k, R}$ is continuous in time (cf. Lemma 2.6), for any $R>0$, we have $\mathbb{P}\left\{\tau_{k, R}>\right.$ $0, \forall k \geq 1\}=1$. Now we let $R=R_{k}$ be discrete and then denote $\left(X_{k}, \tau_{k}\right)=$ $\left(X_{k, R_{k}}, \tau_{k, R_{k}}\right)$. If $R_{k}^{2}>k^{2}+2$, then $\mathbb{P}\left\{\tau_{k}>0, \forall k \geq 1\right\}=1$ and

$$
\mathbb{P}\left\{\left\|X_{k}\right\|_{\mathcal{V}}^{2} \leq\left\|X_{k}\right\|_{\mathcal{X}}^{2} \leq\left\|X_{0, k}\right\|_{\mathcal{X}}^{2}+2<R_{k}^{2}, \forall t \in\left[0, \tau_{k}\right], \forall k \geq 1\right\}=1
$$

which means

$$
\mathbb{P}\left\{\chi_{R_{k}}\left(\left\|X_{k}\right\| \mathcal{V}\right)=1, \forall t \in\left[0, \tau_{k}\right], \forall k \geq 1\right\}=1
$$

Therefore, $\left(X_{k}, \tau_{k}\right)$ is the pathwise solution to (1.1) with initial value $X_{0, k}$. As shown in Lemma 2.4, $\mathbf{1}_{\Omega_{k}} X_{k}$ also solves (1.1) with initial value $X_{0, k}$ on $\left[0, \mathbf{1}_{\Omega_{k}} \tau_{k}\right]$. Then, uniqueness means $X_{k}=\mathbf{1}_{\Omega_{k}} X_{k}$ on $\left[0, \mathbf{1}_{\Omega_{k}} \tau_{k}\right] \mathbb{P}-$ a.s. Therefore, we infer from $\mathbb{P}\left\{\bigcup_{k \geq 1} \Omega_{k}\right\}=1$ that the pair

$$
\left(X=\sum_{k \geq 1} \mathbf{1}_{\Omega_{k}} X_{k}, \quad \tau=\sum_{k \geq 1} \mathbf{1}_{\Omega_{k}} \tau_{k}\right)
$$

is a pathwise solution to (1.1) corresponding to the initial condition $X_{0}$. Since for each $k, X_{k}$ is continuous in time (cf. Lemma 2.6), so is $X$. Then, we have

$$
\sup _{t \in[0, \tau]}\|X\|_{H^{s}}^{2}=\sum_{k \geq 1} \mathbf{1}_{\Omega_{k}} \sup _{t \in\left[0, \tau_{k}\right]}\left\|X_{k}\right\|_{H^{s}}^{2} \leq \sum_{k \geq 1} \mathbf{1}_{\Omega_{k}}\left(\left\|X_{0, k}\right\|_{H^{s}}^{2}+2\right) \leq 2\left\|X_{0}\right\|_{H^{s}}^{2}+4 .
$$

Taking expectation gives rise to (2.21) and we have finished the proof of (ii) in Theorem 2.1 .

\subsection{Proof of (iii) in Theorem 2.1}

To complete the proof of Theorem 2.1, it suffices to prove the blow-up criterion (2.22) when Assumption (C) holds true additionally. To show it, we define

$\tau_{1, m}:=\inf \{t \geq 0:\|X(t)\| \mathcal{X} \geq m\}, \quad \tau_{2, l}:=\inf \{t \geq 0:\|X(t)\| \mathcal{V} \geq l\}, \quad m, l \in \mathbb{N}$, 
where $\inf \emptyset=\infty$. Denote $\tau_{1}=\lim _{m \rightarrow \infty} \tau_{1, m}$ and $\tau_{2}=\lim _{l \rightarrow \infty} \tau_{2, l}$. Then, (2.22) is just a direct consequence of the statement

$$
\tau_{1}=\tau_{2} \mathbb{P}-\text { a.s. }
$$

Hence, it suffices to prove (2.49). Because $\mathcal{X} \hookrightarrow \mathcal{V}$, it is obvious that $\tau_{1} \leq \tau_{2} \mathbb{P}-$ a.s. Therefore, the proof reduces further to checking only $\tau_{1} \geq \tau_{2} \mathbb{P}-$ a.s. We first notice that for all $M, l \in \mathbb{N}$,

$$
\left\{\sup _{t \in\left[0, \tau_{2, l} \wedge M\right]}\|X(t)\| \mathcal{X}<\infty\right\}=\bigcup_{m \in \mathbb{N}}\left\{\sup _{t \in\left[0, \tau_{2, l} \wedge M\right]}\|X(t)\| \mathcal{X}<m\right\} \subset \bigcup_{m \in \mathbb{N}}\left\{\tau_{2, l} \wedge M \leq \tau_{1, m}\right\} .
$$

Because

$$
\bigcup_{m \in \mathbb{N}}\left\{\tau_{2, l} \wedge M \leq \tau_{1, m}\right\} \subset\left\{\tau_{2, l} \wedge M \leq \tau_{1}\right\}
$$

as long as

$$
\mathbb{P}\left(\sup _{t \in\left[0, \tau_{2, l} \wedge M\right]}\|X(t)\| \mathcal{X}<\infty\right)=1 \forall M, l \in \mathbb{N},
$$

we have $\mathbb{P}\left(\tau_{2, l} \wedge M \leq \tau_{1}\right)=1$ for all $M, l \in \mathbb{N}$, and

$$
\mathbb{P}\left(\tau_{2} \leq \tau_{1}\right)=\mathbb{P}\left(\bigcap_{l \in \mathbb{N}}\left\{\tau_{2, l} \leq \tau_{1}\right\}\right)=\mathbb{P}\left(\bigcap_{M, l \in \mathbb{N}}\left\{\tau_{2, l} \wedge M \leq \tau_{1}\right\}\right)=1
$$

As a result, it remains to prove (2.50). However, as mentioned before, we cannot directly apply the Itô formula to $\|X\|_{\mathcal{X}}^{2}$ to get control of $\mathbb{E}\|X(t)\|_{\mathcal{X}}^{2}$. As in (2.46), but now with $Q_{\varepsilon}$, we use Itô formula for $\left\|Q_{\varepsilon} X\right\|_{\mathcal{X}}^{2}$, apply the BDG inequality, $\left(\mathbf{A}_{1}\right)$ and Assumption (C) to find constants $C_{1}>0$ and $C_{2}=C_{2}(l)>0$ such that

$$
\begin{aligned}
& \mathbb{E} \sup _{t \in\left[0, \tau_{2, l} \wedge M\right]}\left\|Q_{\varepsilon} X\right\|_{\mathcal{X}}^{2}-\mathbb{E}\left\|Q_{\varepsilon} X_{0}\right\|_{\mathcal{X}}^{2} \\
& \leq C_{1} \mathbb{E}\left(\int_{0}^{\tau_{2, l} \wedge M} k(t) f\left(\|X\|_{\mathcal{V}}\right)\|X\|_{\mathcal{X}}^{2}\left\|Q_{\varepsilon} X\right\|_{\mathcal{X}}^{2} \mathrm{~d} t\right)^{\frac{1}{2}} \\
& \quad+C_{1} \mathbb{E} \int_{0}^{\tau_{2, l} \wedge M} k(t) f\left(\|X\|_{\mathcal{V}}\right)\|X\|_{\mathcal{X}}^{2} \mathrm{~d} t \\
& \leq C_{2} \mathbb{E}\left(\sup _{t \in\left[0, \tau_{2, l} \wedge M\right]}\left\|Q_{\varepsilon} X\right\|_{\mathcal{X}}^{2} \int_{0}^{\tau_{2, l} \wedge M} k(t)\|X\|_{\mathcal{X}}^{2} \mathrm{~d} t\right)^{\frac{1}{2}}
\end{aligned}
$$




$$
\begin{aligned}
& +C_{2} \mathbb{E} \int_{0}^{\tau_{2, l} \wedge M} k(t)\|X\|_{\mathcal{X}}^{2} \mathrm{~d} t \\
\leq & \frac{1}{2} \mathbb{E} \sup _{t \in\left[0, \tau_{2, l} \wedge M\right]}\left\|Q_{\varepsilon} X\right\|_{\mathcal{X}}^{2}+C_{2} \int_{0}^{M} k(t) \mathbb{E} \sup _{t^{\prime} \in\left[0, t \wedge \tau_{2, l}\right]}\left\|X\left(t^{\prime}\right)\right\|_{\mathcal{X}}^{2} \mathrm{~d} t .
\end{aligned}
$$

This yields

$$
\mathbb{E} \sup _{t \in\left[0, \tau_{2, l} \wedge M\right]}\left\|Q_{\varepsilon} X\right\|_{\mathcal{X}}^{2} \leq 2 \mathbb{E}\left\|X_{0}\right\|_{\mathcal{X}}^{2}+C_{2} \int_{0}^{M} k(t) \mathbb{E} \sup _{t^{\prime} \in\left[0, t \wedge \tau_{2, l}\right]}\left\|X\left(t^{\prime}\right)\right\|_{\mathcal{X}}^{2} \mathrm{~d} t
$$

Since the right hand side of the inequality above does not depend on $\varepsilon$, and since $Q_{\varepsilon}$ satisfies (2.16), we can send $\varepsilon \rightarrow 0$ to find

$$
\mathbb{E} \sup _{t \in\left[0, \tau_{2, l} \wedge M\right]}\|X\|_{\mathcal{X}}^{2} \leq 2 \mathbb{E}\left\|X_{0}\right\|_{\mathcal{X}}^{2}+C_{2} \int_{0}^{M} k(t) \mathbb{E} \sup _{t^{\prime} \in\left[0, t \wedge \tau_{2, l}\right]}\left\|X\left(t^{\prime}\right)\right\|_{\mathcal{X}}^{2} \mathrm{~d} t
$$

Then, Grönwall's inequality shows that for each $l, M \in \mathbb{N}$,

$$
\mathbb{E} \sup _{t \in\left[0, \tau_{2, l} \wedge M\right]}\|X(t)\|_{\mathcal{X}}^{2} \leq 2 \mathbb{E}\left\|X_{0}\right\|_{\mathcal{X}}^{2} \exp \left\{C_{2} \int_{0}^{M} k(t) \mathrm{d} t\right\}<\infty
$$

which gives (2.50). We conclude the proof of (iii) in Theorem 2.1.

\section{Applications to Nonlinear Ideal Fluid Models with Transport Noise}

\subsection{Stochastic Advection by Lie Transport in Fluid Dynamics}

Starting with the pioneering works (Fedrizzi and Flandoli 2013; Flandoli et al. 2010) for linear scalar transport equations, many achievements have been made in recent years for stochastic fluid equations with noise of transport type. Transport-type noise refers to noise depending linearly on the gradient of the solution. In Holm (2015), stochastic equations governing the dynamics of some ideal fluid regimes have been derived by employing a novel variational principle for stochastic Lagrangian particle dynamics. Later, the same stochastic evolution equations were rediscovered in Cotter et al. (2017) using a multi-scale decomposition of the deterministic Lagrangian flow map into a slow large-scale mean, and a rapidly fluctuating small-scale map. In Holm (2015), the extension of geometric mechanics to include stochasticity in nonlinear fluid theories was accomplished by using Hamilton's variational principle. This extension motivates us to study stochastic Lagrangian fluid trajectories, denoted as $X_{t}(x, t)$, arising from the stochastic Eulerian vector field with a noise in the Stratonovich sense, i.e., 


$$
\mathrm{d} X_{t}(x, t):=u(x, t) d t+\sum_{k=1}^{M} \xi_{k}(x) \circ \mathrm{d} W_{k}
$$

In (3.1) $u(x, t)$ means the drift velocity, $\left\{W_{k}=W_{k}(t)\right\}_{k=1,2, \ldots, M}$ is a family of standard 1-D independent Brownian motions, and $M$ can be determined via the amount of variance required from a principal component analysis, or via empirical orthogonal function analysis.

Deriving continuum-scale equations taking into account noise as in (3.1) is known as the Stochastic Advection by Lie Transport (SALT) approach, see Cotter et al. (2019) and the references therein. The SALT approach combines stochasticity in the velocity of the fluid material loop in Kelvin's circulation theorem with ensemble forecasting and meets the important challenge of incorporating stochastic parameterization at the fundamental level, see for example Berner et al. (2012), Leslie and Quarini (1979) and Zidikheri and Frederiksen (2010).

Many subsequent investigations of the properties of the equations of fluid dynamics with the SALT modification have appeared in the literature recently. For example, local existence in Sobolev spaces and a Beale-Kato-Majda type blow-up criterion were derived in Crisan et al. (2019) and Flandoli and Luo (2019) for the incompressible 3-D SALT Euler equations. For the 2-D version, global existence of solutions has been shown in Crisan and Lang (2019). In Alonso-Orán and Bethencourt de León (2020), the authors provide a local existence result for the incompressible 2-D SALT Boussinesq equations. For a simpler but still nonlinear equation as the SALT Burgers equation, we refer to Alonso-Orán et al. (2019) and Flandoli (2011).

\subsubsection{The Two-Component CH System with Transport Noise}

The Camassa-Holm $(\mathrm{CH})$ equation

$$
u_{t}-u_{x x t}+3 u u_{x}=2 u_{x} u_{x x}+u u_{x x x}
$$

was proposed independently by Fuchssteiner and Fokas (1981) and by Camassa and Holm (1993). In Fuchssteiner and Fokas (1981), it was proposed to consider some completely integrable generalizations of the Korteweg-De Vries equation with biHamiltonian structures, and in Camassa and Holm (1993), it was derived to describe the unidirectional propagation of shallow water waves over a flat bottom. Solutions to equation (3.2) exhibit the wave-breaking phenomenon, i.e., smooth global existence may fail (Constantin and Escher 1998a, b). Global conservative solutions to the $\mathrm{CH}$ equation (3.2) were obtained in Bressan and Constantin (2007) and Holden and Raynaud (2007). Different stochastic versions of the $\mathrm{CH}$ equation have been studied including additive noise (Chen et al. 2012) and multiplicative noise (Albeverio et al. 2021; Rohde and Tang 2020, 2021; Tang 2018, 2020). Following the approach in Holm (2015), the corresponding stochastic version of the $\mathrm{CH}$ equation with transport noise was introduced in Bendall et al. (2019) and Crisan and Holm (2018). Transforming the equation into a partial differential equation with random coefficients, the well-posedness of the stochastic $\mathrm{CH}$ equation with some special transport noise has 
been studied in Albeverio et al. (2021). We can extend this result to a far more complex system: the stochastic two-component $\mathrm{CH}$ system (see Holm and Luesink (2021) for the related models), i.e.,

$$
\left\{\begin{array}{l}
\mathrm{d} m+\left(m \partial_{x}+\partial_{x} m\right) \mathrm{d} \chi_{t}+\eta \partial_{x} \eta \mathrm{d} t=0 \\
\mathrm{~d} \eta+\left(\eta \mathrm{d} \chi_{t}\right)_{x}=0 \\
m=u-u_{x x}
\end{array}\right.
$$

In (3.3), $u$ is the fluid velocity and $\eta$ denotes the depth of the flow. As in (3.1), the noise structure in (3.3) is

$$
\mathrm{d} \chi_{t}=u(t, x) \mathrm{d} t+\sum_{k=1}^{M} \xi_{k}(x) \circ \mathrm{d} W_{k} .
$$

The functions $\xi_{1}, \ldots, \xi_{M}$ represent spatial velocity-velocity correlations up to order $M$.

Note that the system (3.3) reduces to the scalar $\mathrm{CH}$ equation from Albeverio et al. (2021) if we put $\eta$ to be zero. Here we consider $M=\infty$ and rewrite (3.3) as

$$
\left\{\begin{array}{l}
\mathrm{d} m+\left[(m u)_{x}+\eta \eta_{x}\right] \mathrm{d} t+\sum_{k=1}^{\infty} \mathcal{L}_{\xi_{k}} m \circ \mathrm{d} W_{k}=0 \\
\mathrm{~d} \eta+(\eta u)_{x} \mathrm{~d} t+\sum_{k=1}^{\infty} \mathcal{L}_{\xi_{k}} \eta \circ \mathrm{d} W_{k}=0 .
\end{array}\right.
$$

The differential operator $\mathcal{L}_{\xi_{k}}$ is given by

$$
\mathcal{L}_{\xi_{k}}=\partial_{x} \xi_{k}+\xi_{k} \partial_{x}
$$

Calculating the cross-variation term in the general transformation formula

$$
\int_{0}^{t} f \circ \mathrm{d} W=\int_{0}^{t} f \mathrm{~d} W+\frac{1}{2}\langle f, W\rangle_{t},
$$

we obtain the corresponding Itô formulation of (3.4), given by

$$
\left\{\begin{array}{l}
\mathrm{d} m+\left[(m u)_{x}+\eta \eta_{x}\right] \mathrm{d} t-\frac{1}{2} \sum_{k=1}^{\infty} \mathcal{L}_{\xi_{k}}^{2} m \mathrm{~d} t=-\sum_{k=1}^{\infty} \mathcal{L}_{\xi_{k}} m \mathrm{~d} W_{k}, \\
\mathrm{~d} \eta+(\eta u)_{x} \mathrm{~d} t-\frac{1}{2} \sum_{k=1}^{\infty} \mathcal{L}_{\xi_{k}}^{2} \eta \mathrm{d} t=-\sum_{k=1}^{\infty} \mathcal{L}_{\xi_{k}} \eta \mathrm{d} W_{k} .
\end{array}\right.
$$

Note that the operator $\mathcal{L}_{\xi_{k}}^{2}$ in (3.6) is the second-order operator

$$
\mathcal{L}_{\xi_{k}}^{2} f=\mathcal{L}_{\xi_{k}}\left(\mathcal{L}_{\xi_{k}} f\right)=\xi_{k}^{2} \partial_{x x}^{2} f+3 \xi_{k} \partial_{x} \xi_{k} \partial_{x} f+\left(\xi_{k} \partial_{x x}^{2} \xi_{k}+\left(\partial_{x} \xi_{k}\right)^{2}\right) f
$$


In this paper, we will consider (3.6) on the periodic torus $\mathbb{T}=\mathbb{R} / 2 \pi \mathbb{Z}$ in terms of the unknowns $(u, \eta)$. Therefore, for any real number $s$, we define $D^{s}=\left(I-\partial_{x x}^{2}\right)^{s / 2}$ as $\widehat{D^{s} f}(k)=\left(1+|k|^{2}\right)^{s / 2} \widehat{f}(k)$. Then, we apply $\left(I-\partial_{x x}^{2}\right)^{-1}=D^{-2}$ to (3.6) and consider for $(u, \eta)$ the nonlocal Cauchy problem

$$
\left\{\begin{array}{l}
\mathrm{d} u+\left[u u_{x}+\partial_{x} D^{-2}\left(\frac{1}{2} u^{2}+u_{x}^{2}+\frac{1}{2} \eta^{2}\right)-\frac{1}{2} D^{-2} \sum_{k=1}^{\infty} \mathcal{L}_{\xi_{k}}^{2} D^{2} u\right] \mathrm{d} t \\
\quad=-D^{-2} \sum_{k=1}^{\infty} \mathcal{L}_{\xi_{k}} D^{2} u \mathrm{~d} W_{k} \\
\mathrm{~d} \eta+\left(u \eta_{x}+\eta u_{x}\right) \mathrm{d} t-\frac{1}{2} \sum_{k=1}^{\infty} \mathcal{L}_{\xi_{k}}^{2} \eta \mathrm{d} t=-\sum_{k=1}^{\infty} \mathcal{L}_{\xi_{k}} \eta \mathrm{d} W_{k}, \\
(u(0), \eta(0))=\left(u_{0}, \eta_{0}\right)
\end{array}\right.
$$

Here we remark that in (3.7), $f=D^{-2} g=\left(I-\partial_{x x}^{2}\right)^{-1} g$ means $f=\mathcal{G} \star g$, where $\mathcal{G}$ is the Green function of the Helmholtz operator $\left(I-\partial_{x x}^{2}\right)$ and $\star$ stands for the convolution. The local theory for (3.7) is stated in Theorem 3.1.

\subsubsection{The CCF Model with Transport Noise}

As the second application of the abstract framework, we will consider a stochastic transport equation with non-local velocity on the periodic torus $\mathbb{T}$. In the deterministic case, it reads

$$
\theta_{t}+(\mathcal{H} \theta) \theta_{x}=0
$$

where $\mathcal{H}$ is the periodic Hilbert transform defined by

$$
(\mathcal{H} f)(x)=\frac{1}{2 \pi} \text { p.v. } \int_{0}^{2 \pi} f(t) \cot \left(\frac{x-t}{2}\right) \mathrm{d} t .
$$

Equation (3.8) was proposed by Córdoba et al. (2005) to consider advective transport with non-local velocity. It is deeply connected to the 2-D SQG equation and hence with the 3-D Euler equations (cf. Bae and Granero-Belinchón 2015 and the references therein). Notice that, if we replace the non-local Hilbert transform by the identity operator we recover the classical Burgers equation. In Córdoba et al. (2005), the breakdown of classical solutions to (3.8) for a generic class of smooth initial data was discovered.

To the best of our knowledge, the stochastic counterpart of the CCF model (3.8) has not been studied yet. In this paper, we will consider the stochastic CCF model with transport noise, i.e.,

$$
\mathrm{d} \theta+(\mathcal{H} \theta) \partial_{x} \theta \mathrm{d} t+\sum_{k=1}^{\infty} \mathcal{L}_{\xi_{k}} \theta \circ \mathrm{d} W_{k}=0,
$$


where $\left\{W_{k}=W_{k}(t)\right\}_{k \in \mathbb{N}}$ is a sequence of standard 1-D independent Brownian motions and $\mathcal{L}_{\xi_{k}}$ is given as in (3.5). Using the corresponding Itô formulation, we are led to the Cauchy problem

$$
\left\{\begin{array}{l}
\mathrm{d} \theta+(\mathcal{H} \theta) \partial_{x} \theta \mathrm{d} t-\frac{1}{2} \sum_{k=1}^{\infty} \mathcal{L}_{\xi_{k}}^{2} \theta \mathrm{d} t=-\sum_{k=1}^{\infty} \mathcal{L}_{\xi_{k}} \theta \mathrm{d} W_{k}, \\
\theta(0)=\theta_{0} .
\end{array}\right.
$$

A local theory for (3.11) is stated in Theorem 3.2.

\subsection{Notations, Assumptions and Main Results}

To state the main results for (3.7) and (3.11), we introduce some function spaces. For $d \in \mathbb{N}$ and $1 \leq p<\infty$ we denote by $L^{p}\left(\mathbb{T}^{d} ; \mathbb{R}\right)$ the standard Lebesgue space of measurable $p$-integrable $\mathbb{R}$-valued functions with domain $\mathbb{T}^{d}=(\mathbb{R} / 2 \pi \mathbb{Z})^{d}$ and by $L^{\infty}\left(\mathbb{T}^{d} ; \mathbb{R}\right)$ the space of essentially bounded functions. Particularly, $L^{2}\left(\mathbb{T}^{d} ; \mathbb{R}\right)$ is equipped with the inner product $(f, g)_{L^{2}}=\int_{\mathbb{T}^{d}} f \cdot \bar{g} \mathrm{~d} x$, where $\bar{g}$ denotes the complex conjugate of $g$. The Fourier transform and inverse Fourier transform of $f(x) \in L^{2}\left(\mathbb{T}^{d} ; \mathbb{R}\right)$ are defined by $\widehat{f}(\xi)=\int_{\mathbb{T}^{d}} f(x) \mathrm{e}^{-\mathrm{i} x \cdot \xi} \mathrm{d} x$ and $f(x)=$ $\frac{1}{(2 \pi)^{d}} \sum_{k \in \mathbb{Z}^{d}} \widehat{f}(k) \mathrm{e}^{\mathrm{i} x \cdot k}$, respectively. Recalling that for any $s \in \mathbb{R}, \widehat{D^{s} f}(k)=$ $\left(1+|k|^{2}\right)^{s / 2} \widehat{f}(k)$, we define the Sobolev space $H^{s}$ on $\mathbb{T}^{d}$ with values in $\mathbb{R}$ as

$$
H^{s}\left(\mathbb{T}^{d} ; \mathbb{R}\right):=\left\{f \in L^{2}\left(\mathbb{T}^{d} ; \mathbb{R}\right):\|f\|_{H^{s}\left(\mathbb{T}^{d} ; \mathbb{R}\right)}^{2}=\sum_{k \in \mathbb{Z}^{d}}\left|\widehat{D^{s} f}(k)\right|^{2}<+\infty\right\}
$$

For $u=\left(u_{j}\right)_{1 \leq j \leq n}: \mathbb{T}^{d} \mapsto \mathbb{R}^{n}$, we define $\|u\|_{H^{s}\left(\mathbb{T}^{d} ; \mathbb{R}^{n}\right)}^{2}:=\sum_{j=1}^{n}\left\|u_{j}\right\|_{H^{s}\left(\mathbb{T}^{d} ; \mathbb{R}\right)}^{2}$. For the sake of simplicity, we omit the parentheses in the above notations from now on if there is no ambiguity. Similarly, for two spaces $H^{s_{1}}$ and $H^{s_{2}}\left(s_{1}, s_{2}>0\right)$ and $(f, g) \in H^{s_{1}} \times H^{s_{2}}$, we define $\|(f, g)\|_{H^{s_{1}} \times H^{s_{2}}}^{2}:=\|f\|_{H^{s_{1}}}^{2}+\|g\|_{H^{s_{2}}}^{2}$. The commutator for two operators $P, Q$ is denoted by $[P, Q]:=P Q-Q P$. The space of bounded linear operators from $\mathbb{U}$ to some separable Hilbert space $\mathbb{X}$ is denoted by $\mathcal{L}(\mathbb{U} ; \mathbb{X})$.

To obtain a local theory for (3.7) and (3.11), we have to impose natural regularity assumptions on $\left\{\xi_{k}(x)\right\}_{k \in \mathbb{N}}$ to give a reasonable meaning to the stochastic integral and to show certain estimates. For this reason, we make the following assumption:

Assumption (D) $\sum_{k \in \mathbb{N}}\left\|\xi_{k}\right\|_{H^{s}}<\infty$ for any $s \geq 0$.

Remark 3.1 It follows from Assumption (D) that there is a $C>0$ such that for all $f \in H^{s+2}$ with $s>\frac{1}{2}$, we have

$$
\sum_{k=1}^{\infty}\left\|\mathcal{L}_{\xi_{k}} f\right\|_{H^{s}} \leq C\|f\|_{H^{s+1}} \text { and } \sum_{k=1}^{\infty}\left\|\mathcal{L}_{\xi_{k}}^{2} f\right\|_{H^{s}} \leq C\|f\|_{H^{s+2}}
$$


Besides, we do not require that $\left\{\xi_{k}\right\}_{k \in \mathbb{N}}$ is an orthogonal system.

The main results for (3.7) and (3.11) are the following:

Theorem 3.1 Let $s>\frac{11}{2}$ and $\mathcal{S}=\left(\Omega, \mathcal{F}, \mathbb{P},\left\{\mathcal{F}_{t}\right\}_{t \geq 0}, \mathcal{W}\right)$ be a stochastic basis fixed in advance. Let Assumption (D) hold. If $\left(u_{0}, \eta_{0}\right) \in L^{2}\left(\Omega ; H^{s} \times H^{s-1}\right)$ is an $\mathcal{F}_{0}$-measurable random variable, then (3.7) has a local unique pathwise solution $((u, \eta), \tau)$ such that

$$
(u, \eta)(\cdot \wedge \tau) \in L^{2}\left(\Omega ; C\left([0, \infty) ; H^{s} \times H^{s-1}\right)\right) .
$$

Moreover, the maximal solution $\left((u, \eta), \tau^{*}\right)$ to (3.7) satisfies

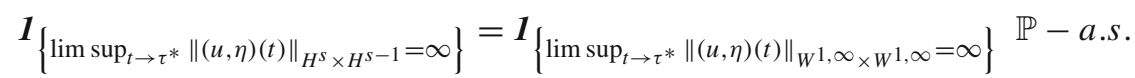

Theorem 3.2 Let $s>\frac{7}{2}$ and $\mathcal{S}=\left(\Omega, \mathcal{F}, \mathbb{P},\left\{\mathcal{F}_{t}\right\}_{t \geq 0}, \mathcal{W}\right)$ be a stochastic basis fixed in advance. Let Assumption (D) hold. If $\theta_{0} \in L^{2}\left(\Omega ; H^{s}\right)$ is an $\mathcal{F}_{0}$-measurable random variable, then (3.11) has a local unique pathwise solution $(\theta, \tau)$ such that

$$
\theta(\cdot \wedge \tau) \in L^{2}\left(\Omega ; C\left([0, \infty) ; H^{s}\right)\right)
$$

Moreover, the maximal solution $\left(\theta, \tau^{*}\right)$ to $(3.11)$ satisfies

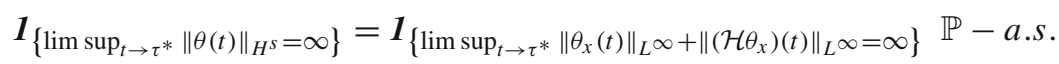

Remark 3.2 We require $s>11 / 2$ in Theorem 3.1. This is because, if $(u, \eta) \in H^{s} \times$ $H^{s-1}$, then $\left(-\frac{1}{2} D^{-2} \sum_{k=1}^{\infty} \mathcal{L}_{\xi_{k}}^{2} D^{2} u,-\frac{1}{2} \sum_{k=1}^{\infty} \mathcal{L}_{\xi_{k}}^{2} \eta\right) \in H^{s-2} \times H^{s-3}$. To apply Theorem 2.1 to (3.7) with $\mathcal{X}=H^{s} \times H^{s-1}$, we have to verify (2.15) with using Lemma A.5. Therefore, $s-4>\frac{3}{2}$, which means $s>11 / 2$. Similarly, $s>7 / 2$ is needed in Theorem 3.2.

Remark 3.3 The scalar stochastic $\mathrm{CH}$ equation with transport noise has been analyzed very recently in Albeverio et al. (2021) with a completely different approach. The authors obtain the local existence of pathwise solutions in less regular spaces but without a blow-up criterion. Note that the abstract framework developed in this article can be applied to cover this equation to show local existence, uniqueness and derive a blow-up criterion.

Remark 3.4 Notice that in the deterministic case, one can use the estimate

$$
\left\|\mathcal{H} \theta_{x}\right\|_{L^{\infty}} \lesssim\left(1+\left\|\theta_{x}\right\|_{L^{\infty}} \log \left(\mathrm{e}+\left\|\theta_{x}\right\|_{H^{1}}\right)+\left\|\theta_{x}\right\|_{L^{2}}\right)
$$

to improve the blow-up criterion (3.14) into (cf. Dong 2008)

$$
\limsup _{t \rightarrow \tau^{*}}\|\theta(t)\|_{H^{s}}=\infty \Longleftrightarrow \limsup _{t \rightarrow \tau^{*}}\left\|\theta_{x}(t)\right\|_{L^{\infty}}=\infty .
$$


To achieve this in the stochastic setting, we have an essential difficulty in closing the $H^{s}$-estimate. That is, one has to split the expectation $\mathbb{E}\left\|\mathcal{H} \theta_{x}\right\|_{L^{\infty}}\|\theta\|_{H^{s}}^{2}$. By invoking (3.15), so far we have not been able to close the estimate for $\mathbb{E}\|\theta\|_{H^{s}}^{2}$ because $\mathbb{E}\left[\left(1+\left\|\theta_{x}\right\|_{L^{\infty}} \log \left(\mathrm{e}+\left\|\theta_{x}\right\|_{H^{1}}\right)+\left\|\theta_{x}\right\|_{L^{2}}\right)\|\theta\|_{H^{s}}\right]$ is involved.

\subsection{The Stochastic Two-Component CH System: Proof of Theorem 3.1}

Now we consider (3.7) on the periodic torus $\mathbb{T}$, and we will apply the abstract framework developed in Sect. 2 to obtain Theorem 3.1. To put (3.7) into the abstract framework, we define

$$
X=(u, \eta), G(u, \eta)=\partial_{x} D^{-2}\left(\frac{1}{2} u^{2}+u_{x}^{2}+\frac{1}{2} \eta^{2}\right)
$$

and we set

$$
\left\{\begin{aligned}
b(t, X) & =b(X)=\left(-G(u, \eta),-\eta u_{X}\right), \\
g(t, X) & =g(X)=\left(-u u_{x}+\frac{1}{2} D^{-2} \sum_{k=1}^{\infty} \mathcal{L}_{\xi_{k}}^{2} D^{2} u,-u \eta_{x}+\frac{1}{2} \sum_{k=1}^{\infty} \mathcal{L}_{\xi_{k}}^{2} \eta\right), \\
h^{k}(t, X) & =h^{k}(X)=\left(-D^{-2} \mathcal{L}_{\xi_{k}} D^{2} u,-\mathcal{L}_{\xi_{k}} \eta\right), \quad k \in \mathbb{N} .
\end{aligned}\right.
$$

Now we recall that $\mathbb{U}$ is a fixed separable Hilbert space and $\left\{e_{i}\right\}_{i \in \mathbb{N}}$ is a complete orthonormal basis of $\mathbb{U}$ such that the cylindrical Wiener process $\mathcal{W}$ is defined as in (2.1). Then, we define $h(X) \in \mathcal{L}\left(\mathbb{U} ; H^{s} \times H^{s-1}\right)$ such that

$$
h(X)\left(e_{k}\right)=h^{k}(X)=\left(-D^{-2} \mathcal{L}_{\xi_{k}} D^{2} u,-\mathcal{L}_{\xi_{k}} \eta\right), \quad k \in \mathbb{N} .
$$

Altogether we can rewrite the problem (3.7) as

$$
\left\{\begin{array}{l}
\mathrm{d} X=(b(X)+g(X)) \mathrm{d} t+h(X) \mathrm{d} \mathcal{W}, \\
X(0)=X_{0}=\left(u_{0}, \eta_{0}\right)
\end{array}\right.
$$

In order to prove Theorem 3.1 by applying Theorem 2.1 , we need to check that Assumptions (A), (B) and (C) are satisfied. To ease notation, we define

$$
\mathcal{X}^{s}=H^{s} \times H^{s-1}
$$

and make the following choice for the spaces $\mathcal{X} \subset \mathcal{Y} \subset \mathcal{Z}$ and $\mathcal{Z} \subset \mathcal{V}$,

$$
\mathcal{X}=\mathcal{X}^{s}, \mathcal{Y}=\mathcal{X}^{s-1}, \mathcal{Z}=\mathcal{X}^{s-2}, \mathcal{V}=W^{1, \infty} \times W^{1, \infty}
$$




\subsubsection{Estimates on Nonlinear Terms}

In this preparatory part, some basic Sobolev estimates to deal with $b, g, h$ from (3.16), (3.17) are introduced.

Lemma 3.1 Let $s>5 / 2$. Then, $b$ is regular in $\mathcal{X}^{s}$ and for $X=(u, \eta) \in \mathcal{X}^{s}, Y=$ $(v, \rho) \in \mathcal{X}^{s}$, we have

$$
\begin{aligned}
\|b(X)\| \mathcal{X}^{s} & \lesssim\|X\|_{\mathcal{V}\|X\|_{\mathcal{X}^{s}}} \\
\|b(X)-b(Y)\|_{\mathcal{X}^{s}} & \lesssim\left(\|X\|_{\mathcal{X}^{s}}+\|Y\|_{\mathcal{X}^{s}}\right)\|X-Y\|_{\mathcal{X}^{s}}
\end{aligned}
$$

Proof Since $\partial_{x}\left(1-\partial_{x x}^{2}\right)^{-1}$ is a bounded map from $H^{s}$ to $H^{s+1}$, the first estimate follows from

$$
\begin{aligned}
\|b(X)\|_{\mathcal{X}^{s}}^{2} & =\|G(u, \eta)\|_{H^{s}}^{2}+\left\|u_{x} \eta\right\|_{H^{s-1}}^{2} \\
& \lesssim\left\|u^{2}+u_{x}^{2}+\eta\right\|_{H^{s-1}}^{2}+\left\|u_{x}\right\|_{L^{\infty}}^{2}\|\eta\|_{H^{s-1}}^{2}+\left\|u_{x}\right\|_{H^{s-1}}^{2}\|\eta\|_{L^{\infty}}^{2} \\
& \lesssim\|u\|_{W^{1, \infty}}^{2}\|u\|_{H^{s}}^{2}+\|\eta\|_{L^{\infty}}^{2}\|\eta\|_{H^{s-1}}^{2}+\|u\|_{W^{1, \infty}}^{2}\|\eta\|_{H^{s-1}}^{2}+\|u\|_{H^{s}}^{2}\|\eta\|_{L^{\infty}}^{2} \\
& \lesssim\|(u, \eta)\|_{W^{1, \infty} \times L^{\infty}}^{2}\|(u, \eta)\|_{H^{s} \times H^{s-1}}^{2} .
\end{aligned}
$$

Using the fact that $H^{s-1}$ is an algebra, we can infer that

$$
\begin{aligned}
& \|b(X)-b(Y)\|_{\mathcal{X}^{s}}^{2} \\
& \quad \lesssim\|G(u, \eta)-G(v, \rho)\|_{H^{s}}^{2}+\left\|u_{x} \eta-v_{x} \rho\right\|_{H^{s-1}}^{2} \\
& \quad \lesssim\left\|u^{2}-v^{2}+u_{x}^{2}-v_{x}^{2}+\eta^{2}-\rho^{2}\right\|_{H^{s-1}}^{2}+\left\|u_{x}(\eta-\rho)+\rho\left(u_{x}-v_{x}\right)\right\|_{H^{s-1}}^{2} \\
& \quad \lesssim\|u+v\|_{H^{s}}^{2}\|u-v\|_{H^{s}}^{2}+\|\eta+\rho\|_{H^{s-1}}^{2}\|\eta-\rho\|_{H^{s-1}}^{2} \\
& \quad+\|u\|_{H^{s}}^{2}\|\eta-\rho\|_{H^{s-1}}^{2}+\|\rho\|_{H^{s-1}}^{2}\|u-v\|_{H^{s}}^{2} \\
& \quad \lesssim\left(\|(u, \eta)\|_{H^{s} \times H^{s-1}}^{2}+\|(v, \rho)\|_{H^{s} \times H^{s-1}}^{2}\right)\|(u-v, \eta-\rho)\|_{H^{s} \times H^{s-1}}^{2},
\end{aligned}
$$

which gives the second estimate.

Lemma 3.2 Let Assumption (D) hold true and $s>7 / 2$. If $X=(u, \eta) \in \mathcal{X}^{s}$, then $g: \mathcal{X}^{s} \rightarrow \mathcal{X}^{s-2}$ and $h: \mathcal{X}^{s} \rightarrow \mathcal{L}_{2}\left(\mathbb{U} ; \mathcal{X}^{s-1}\right)$ obey

$$
\|g(X)\|_{\mathcal{X}^{s-2}} \lesssim 1+\|X\|_{\mathcal{X}^{s}}^{2}
$$

and

$$
\|h(X)\|_{\mathcal{L}_{2}\left(\mathbb{U} ; \mathcal{X}^{s-1}\right)} \lesssim\|X\| \mathcal{X}^{s}
$$

Proof Using $H^{s-3} \hookrightarrow L^{\infty}$, we derive 


$$
\begin{aligned}
\|g(X)\|_{\mathcal{X}^{s-2}}^{2}= & \left\|-u u_{x}+\frac{1}{2} D^{-2} \sum_{k=1}^{\infty} \mathcal{L}_{\xi_{k}}^{2} D^{2} u\right\|_{H^{s-2}}^{2} \\
& +\left\|-u \eta_{x}+\frac{1}{2} \sum_{k=1}^{\infty} \mathcal{L}_{\xi_{k}}^{2} \eta\right\|_{H^{s-3}}^{2} \\
& \lesssim\|u\|_{H^{s}}^{4}+\left\|D^{2} u\right\|_{H^{s-2}}^{2}+\|\eta\|_{H^{s-1}}^{2}\|u\|_{H^{s}}^{2}+\|\eta\|_{H^{s-1}}^{2} \\
& \lesssim\left(1+\|u\|_{H^{s}}^{2}+\|\eta\|_{H^{s-1}}^{2}\right)^{2},
\end{aligned}
$$

which implies the first estimate. Similarly, from the definition of $h$ in (3.17), and the definition of $\mathcal{L}_{\xi}$ in (3.5), one has

$$
\begin{aligned}
\sum_{k=1}^{\infty}\left\|h(X) e_{k}\right\|_{\mathcal{X}^{s-1}}^{2} & =\sum_{k=1}^{\infty}\left(\left\|D^{-2} \mathcal{L}_{\xi_{k}} D^{2} u\right\|_{H^{s-1}}^{2}+\left\|\mathcal{L}_{\xi_{k}} \eta\right\|_{H^{s-2}}^{2}\right) \\
& \lesssim \sum_{k=1}^{\infty}\left(\left\|\mathcal{L}_{\xi_{k}} D^{2} u\right\|_{H^{s-3}}^{2}+\left\|\mathcal{L}_{\xi_{k}} \eta\right\|_{H^{s-2}}^{2}\right) \lesssim\|u\|_{H^{s}}^{2}+\|\eta\|_{H^{s-1}}^{2}
\end{aligned}
$$

which gives the second estimate.

Lemma 3.3 Let $s>\frac{11}{2}, X=(u, \eta) \in \mathcal{X}^{s}$ and $Y=(v, \rho) \in \mathcal{X}^{s}$. Then, we have

$$
\begin{aligned}
& 2(g(t, X)-g(t, Y), X-Y)_{\mathcal{X}^{s-2}}+\|h(t, X)-h(t, Y)\|_{\mathcal{L}_{2}\left(\mathbb{U} ; \mathcal{X}^{s-2}\right)}^{2} \\
& \quad \lesssim\left(1+\|X\|_{\mathcal{X}^{s}}^{2}+\|Y\|_{\mathcal{X}^{s}}^{2}\right)\|X-Y\|_{\mathcal{X}^{s-2}}^{2} .
\end{aligned}
$$

Proof Recalling (3.16) and (3.17), we have

$$
\begin{aligned}
2(g(X)-g(Y), X-Y)_{\mathcal{X}^{s-2}}+\|h(t, X)-h(t, Y)\|_{\mathcal{L}_{2}\left(\mathbb{U} ; \mathcal{X}^{s-2}\right)}^{2} \\
=2\left(v v_{x}-u u_{x}, u-v\right)_{H^{s-2}}+2\left(v \rho_{x}-u \eta_{x}, \eta-\rho\right)_{H^{s-3}} \\
\quad+\left(D^{-2} \sum_{k=1}^{\infty} \mathcal{L}_{\xi_{k}}^{2} D^{2}(u-v), u-v\right)_{H^{s-2}} \\
\quad+\sum_{k=1}^{\infty}\left(D^{-2} \mathcal{L}_{\xi_{k}} D^{2}(u-v), D^{-2} \mathcal{L}_{\xi_{k}} D^{2}(u-v)\right)_{H^{s-2}} \\
\quad+\left(\sum_{k=1}^{\infty} \mathcal{L}_{\xi_{k}}^{2}(\eta-\rho), \eta-\rho\right)_{H^{s-3}}
\end{aligned}
$$




$$
\begin{aligned}
& +\sum_{k=1}^{\infty}\left(\mathcal{L}_{\xi_{k}}(\eta-\rho), \mathcal{L}_{\xi_{k}}(\eta-\rho)\right)_{H^{s-3}} \\
:= & \sum_{i=1}^{6} I_{i} .
\end{aligned}
$$

Because $H^{s-2} \hookrightarrow W^{1, \infty}$, we can use Lemma A.4 and integration by parts to arrive at

$$
\begin{aligned}
\left|I_{1}\right| & \lesssim\left|\left(D^{s-2} v(u-v)_{x}, D^{s-2}(u-v)\right)_{L^{2}}\right|+\left|\left(D^{s-2}(u-v) u_{x}, D^{s-2}(u-v)\right)_{L^{2}}\right| \\
& \lesssim\left\|\left[D^{s-2}, v\right](u-v)_{x}\right\|_{L^{2}}\|u-v\|_{H^{s-2}}+\left\|u_{x}\right\|_{L^{\infty}}\|u-v\|_{H^{s-2}}^{2} \\
& \lesssim\left(\|v\|_{H^{s}}+\|u\|_{H^{s}}\right)\|u-v\|_{H^{s-2}}^{2} .
\end{aligned}
$$

Similarly, we have

$$
\begin{aligned}
\left|I_{2}\right| & \lesssim\left|\left(D^{s-3} v(\eta-\rho)_{x}, D^{s-3}(\eta-\rho)\right)_{L^{2}}\right|+\left|\left(D^{s-3}(u-v) \eta_{x}, D^{s-3}(\eta-\rho)\right)_{L^{2}}\right| \\
& \lesssim\left\|\left[D^{s-3}, v\right](\eta-\rho)_{x}\right\|_{L^{2}}\|\eta-\rho\|_{H^{s-3}}+\left\|\eta_{x}\right\|_{H^{s-3}}\|u-v\|_{H^{s-3}}\|\eta-\rho\|_{H^{s-3}} \\
& \lesssim\|v\|_{H^{s}}\|\eta-\rho\|_{H^{s-3}}^{2}+\|\eta\|_{H^{s-1}}^{2}\|u-v\|_{H^{s-3}}^{2}+\|\eta-\rho\|_{H^{s-3}}^{2} .
\end{aligned}
$$

Therefore,

$$
\begin{aligned}
\left|I_{1}\right|+\left|I_{2}\right| & \lesssim\left(\|\eta\|_{H^{s-1}}^{2}+\|v\|_{H^{s}}+\|u\|_{H^{s}}\right)\|u-v\|_{H^{s-2}}^{2}+\left(1+\|v\|_{H^{s}}\right)\|\eta-\rho\|_{H^{s-3}}^{2} \\
& \lesssim\left(1+\|X\|_{\mathcal{X}^{s}}^{2}+\|Y\|_{\mathcal{X}^{s}}^{2}\right)\|X-Y\|_{\mathcal{X}^{s-2}}^{2} .
\end{aligned}
$$

Observe that $D^{s-2} D^{-2}=D^{s-4}$. Since $s-4>3 / 2$, we can invoke Lemma A.5 to obtain

$$
\begin{aligned}
I_{3} & +I_{4} \\
= & \left(D^{s-4} \sum_{k=1}^{\infty} \mathcal{L}_{\xi_{k}}^{2} D^{2}(u-v), D^{s-4} D^{2}(u-v)\right)_{L^{2}} \\
& +\sum_{k=1}^{\infty}\left(D^{s-4} \mathcal{L}_{\xi_{k}} D^{2}(u-v), D^{s-4} \mathcal{L}_{\xi_{k}} D^{2}(u-v)\right)_{L^{2}} \\
& \lesssim\left\|D^{2}(u-v)\right\|_{H^{s-4}}^{2} \lesssim\|u-v\|_{H^{s-2}}^{2} .
\end{aligned}
$$

In the same way, we have 


$$
\begin{aligned}
I_{5} & +I_{6} \\
= & \left(D^{s-3} \sum_{k=1}^{\infty} \mathcal{L}_{\xi_{k}}^{2}(\eta-\rho), D^{s-3}(\eta-\rho)\right)_{L^{2}} \\
& +\sum_{k=1}^{\infty}\left(D^{s-2} \mathcal{L}_{\xi_{k}}(\eta-\rho), D^{s-2} \mathcal{L}_{\xi_{k}}(\eta-\rho)\right)_{L^{2}} \\
& \lesssim\|\eta-\rho\|_{H^{s-3}}^{2} .
\end{aligned}
$$

Collecting the above estimates, we obtain the desired result.

\subsubsection{Proof of Theorem 3.1}

Now we will prove that all the requirements in Assumptions (A), (B) and (C) hold true. We first fix regular mappings $g_{\varepsilon}$ and $h_{\varepsilon}$ using the mollification operators from (A.1) and (A.2) in Appendix A by

$$
\begin{aligned}
g_{\varepsilon}(X)= & \left(-J_{\varepsilon}\left[J_{\varepsilon} u J_{\varepsilon} u_{x}\right]+\frac{1}{2} J_{\varepsilon}^{3} D^{-2} \sum_{k=1}^{\infty} \mathcal{L}_{\xi_{k}}^{2} D^{2} J_{\varepsilon} u,\right. \\
& \left.-J_{\varepsilon}\left[J_{\varepsilon} u J_{\varepsilon} \eta_{x}\right]+\frac{1}{2} J_{\varepsilon}^{3} \sum_{k=1}^{\infty} \mathcal{L}_{\xi_{k}}^{2} J_{\varepsilon} \eta\right) .
\end{aligned}
$$

Let

$$
h_{\varepsilon}^{k}(X)=\left(-J_{\varepsilon} D^{-2} \mathcal{L}_{\xi_{k}} D^{2} J_{\varepsilon} u,-J_{\varepsilon} \mathcal{L}_{\xi_{k}} J_{\varepsilon} \eta\right)
$$

Similar to (3.17), here we define $h_{\varepsilon}(X) \in \mathcal{L}\left(\mathbb{U} ; \mathcal{X}^{s}\right)$ such that

$$
h_{\varepsilon}(X)\left(e_{k}\right)=h_{\varepsilon}^{k}(X), \quad k \in \mathbb{N} \text {. }
$$

In the following, we will show that all the requirements in Assumptions (A), (B) and $(\mathrm{C})$ hold true for the following choice:

$-\mathcal{X}:=\mathcal{X}^{s}, \mathcal{Y}:=\mathcal{X}^{s-1}$ and $\mathcal{Z}:=\mathcal{X}^{s-2}$, where $\mathcal{X}^{s}$ is given in (3.19), and $\mathcal{V}=$ $W^{1, \infty} \times W^{1, \infty}$

$-b, g, h, g_{\varepsilon}$ and $h_{\varepsilon}$ are given in (3.16), (3.17), (3.21) and (3.23), respectively;

$-k(\cdot) \equiv 1, f(\cdot)=C(1+\cdot), q(\cdot)=C\left(1+\cdot^{5}\right)$ for some $C>1$ large enough depending only on $b, g, h$;

$-T_{\varepsilon}=Q_{\varepsilon}=\tilde{J}_{\varepsilon}$, where $\tilde{J}_{\varepsilon}$ is given in (A.2).

Let $s>11 / 2$. Obviously, $\mathcal{X} \hookrightarrow \mathcal{Y} \hookrightarrow \hookrightarrow \mathcal{Z} \hookrightarrow \mathcal{V}$. Then, Lemma 3.1 shows $b: \mathcal{X}^{s} \rightarrow \mathcal{X}^{s}$, and Lemma 3.2 implies $g: \mathcal{X}^{s} \rightarrow \mathcal{X}^{s-2}$ and $h: \mathcal{X}^{s} \rightarrow \mathcal{L}_{2}\left(\mathbb{U} ; \mathcal{X}^{s-1}\right)$. Hence, the stochastic integral in (3.18) is a well defined $\mathcal{X}^{s-1}$-valued local martingale. It is straightforward to verify that all of them are continuous in $X \in \mathcal{X}^{s}$.

Checking ( $\left.\mathbf{A}_{1}\right)$ in Assumption (A): Lemma 3.1 implies $\left(\mathbf{A}_{1}\right)$. 
Checking ( $\left.\mathbf{A}_{2}\right)$ in Assumption (A): By the construction of $g_{\varepsilon}(\cdot)$ and $h_{\varepsilon}(\cdot),(\mathrm{A} .4)$, Lemma 3.2 and Assumption (D), it is easy to check that $\left(\mathbf{A}_{2}\right)$ is satisfied. have

Checking $\left(\mathbf{A}_{3}\right)$ in Assumption (A): We first verify (2.12). By (3.22) and (A.6), we

$$
\begin{aligned}
\left(h_{\varepsilon}^{k}(X), X\right)_{\mathcal{X}^{s}} & =\left(-J_{\varepsilon} D^{-2} \mathcal{L}_{\xi_{k}} D^{2} J_{\varepsilon} u, u\right)_{H^{s}}+\left(-J_{\varepsilon} \mathcal{L}_{\xi_{k}} J_{\varepsilon} \eta, \eta\right)_{H^{s-1}} \\
& =-\left(D^{-2} \mathcal{L}_{\xi_{k}} D^{2} J_{\varepsilon} u, J_{\varepsilon} u\right)_{H^{s}}-\left(\mathcal{L}_{\xi_{k}} J_{\varepsilon} \eta, J_{\varepsilon} \eta\right)_{H^{s-1}} \\
& =-\left(D^{s-2} \mathcal{L}_{\xi_{k}} D^{2} J_{\varepsilon} u, D^{s-2} D^{2} J_{\varepsilon} u\right)_{L^{2}}-\left(D^{s-1} \mathcal{L}_{\xi_{k}} J_{\varepsilon} \eta, D^{s-1} J_{\varepsilon} \eta\right)_{L^{2}} .
\end{aligned}
$$

Let $v=D^{2} J_{\varepsilon} u$. From the definition of the operator $\mathcal{L}_{\xi}$ in (3.5), we have

$$
\begin{aligned}
\left(D^{s-2} \mathcal{L}_{\xi_{k}} v, D^{s-2} v\right)_{L^{2}}= & \left(D^{s-2}\left(v \partial_{x} \xi_{k}\right), D^{s-2} v\right)_{L^{2}}+\left(D^{s-2}\left(\partial_{x} v \xi_{k}\right), D^{s-2} v\right)_{L^{2}} \\
= & \left(\left[D^{s-2}, v\right] \partial_{x} \xi_{k}, D^{s-2} v\right)_{L^{2}}+\left(v D^{s-2} \partial_{x} \xi_{k}, D^{s-2} v\right)_{L^{2}} \\
& +\left(\left[D^{s-2}, \xi_{k}\right] \partial_{x} v, D^{s-2} v\right)_{L^{2}}+\left(\xi_{k} D^{s-2} \partial_{x} v, D^{s-2} v\right)_{L^{2}}
\end{aligned}
$$

By Lemma A.4, $H^{s-2} \hookrightarrow W^{1, \infty}$ and integration by parts, we arrive at

$$
\left(\left[D^{s-2}, v\right] \partial_{x} \xi_{k}, D^{s-2} v\right)_{L^{2}}+\left(v D^{s-2} \partial_{x} \xi_{k}, D^{s-2} v\right)_{L^{2}} \lesssim\|v\|_{H^{s-2}}^{2}\left\|\xi_{k}\right\|_{H^{s-1}}
$$

and

$$
\left(\left[D^{s-2}, \xi_{k}\right] \partial_{x} v, D^{s-2} v\right)_{L^{2}}+\left(\xi_{k} D^{s-2} \partial_{x} v, D^{s-2} v\right)_{L^{2}} \lesssim\|v\|_{H^{s-2}}^{2}\left\|\xi_{k}\right\|_{H^{s-2}}
$$

Combining the above estimates and using (A.7), we have that

$$
\left(D^{s-2} \mathcal{L}_{\xi_{k}} D^{2} J_{\varepsilon} u, D^{s-2} D^{2} J_{\varepsilon} u\right)_{L^{2}} \lesssim\|v\|_{H^{s-2}}^{2}\left\|\xi_{k}\right\|_{H^{s}} \leq\|u\|_{H^{s}}^{2}\left\|\xi_{k}\right\|_{H^{s}}
$$

Similarly,

$$
\left(D^{s-1} \mathcal{L}_{\xi_{k}} J_{\varepsilon} \eta, D^{s-1} J_{\varepsilon} \eta\right)_{L^{2}} \lesssim\left\|J_{\varepsilon} \eta\right\|_{H^{s-1}}^{2}\left\|\xi_{k}\right\|_{H^{s}} \leq\|\eta\|_{H^{s-1}}^{2}\left\|\xi_{k}\right\|_{H^{s}}
$$

Therefore, by using (3.22), (3.23), Assumption (D) and (A.7), we conclude that

$$
\begin{aligned}
\sum_{k=1}^{\infty}\left|\left(h_{\varepsilon}(X) e_{k}, X\right) \mathcal{X}^{s}\right|^{2} & =\sum_{k=1}^{\infty}\left|\left(h_{\varepsilon}^{k}(X), X\right)_{\mathcal{X}^{s}}\right|^{2} \\
& \lesssim \sum_{k=1}^{\infty}\left\|\xi_{k}\right\|_{H^{s}}^{2}\left(\|u\|_{H^{s}}^{2}+\|\eta\|_{H^{s-1}}^{2}\right)^{2} \leq C\|X\|_{\mathcal{X}^{s}}^{4}
\end{aligned}
$$

which yields (2.12). 
Now we prove (2.13). This is the cancellation property. For all $X=(u, \eta) \in \mathcal{X}^{s}$, we have

$$
\begin{aligned}
2\left(g_{\varepsilon}\right. & (X), X)_{\mathcal{X}^{s}}+\left\|h_{\varepsilon}(X)\right\|_{\mathcal{L}_{2}\left(\mathbb{U} ; \mathcal{X}^{s}\right)}^{2} \\
= & -2\left(J_{\varepsilon}\left[J_{\varepsilon} u J_{\varepsilon} u_{x}\right], u\right)_{H^{s}}-2\left(J_{\varepsilon}\left[J_{\varepsilon} u J_{\varepsilon} \eta_{x}\right], \eta\right)_{H^{s-1}} \\
& +\left(D^{s} J_{\varepsilon}^{3} D^{-2} \sum_{k=1}^{\infty} \mathcal{L}_{\xi_{k}}^{2} D^{2} J_{\varepsilon} u, D^{s} u\right)_{L^{2}} \\
& +\sum_{k=1}^{\infty}\left(D^{s} J_{\varepsilon} D^{-2} \mathcal{L}_{\xi_{k}} D^{2} J_{\varepsilon} u, D^{s} J_{\varepsilon} D^{-2} \mathcal{L}_{\xi_{k}} D^{2} J_{\varepsilon} u\right)_{L^{2}} \\
& +\left(D^{s-1} J_{\varepsilon}^{3} \sum_{k=1}^{\infty} \mathcal{L}_{\xi_{k}}^{2} J_{\varepsilon} \eta, D^{s-1} \eta\right)_{L^{2}} \\
& +\sum_{k=1}^{\infty}\left(D^{s-1} J_{\varepsilon} \mathcal{L}_{\xi_{k}} J_{\varepsilon} \eta, D^{s-1} J_{\varepsilon} \mathcal{L}_{\xi_{k}} J_{\varepsilon} \eta\right)_{L^{2}} \\
:= & \sum_{i=1}^{6} E_{i} .
\end{aligned}
$$

It follows from (A.5), (A.7), Lemma A.4 and integration by parts that $\left|E_{1}\right|=2\left|\left(\left[D^{s}, J_{\varepsilon} u\right] J_{\varepsilon} u_{x}, D^{s} J_{\varepsilon} u\right)_{L^{2}}+\left(J_{\varepsilon} u D^{s} J_{\varepsilon} u_{x}, D^{s} J_{\varepsilon} u\right)_{L^{2}}\right| \lesssim\left\|u_{x}\right\|_{L^{\infty}}\|u\|_{H^{s}}^{2}$ and

$$
\begin{aligned}
\left|E_{2}\right| & =2\left|\left(\left[D^{s-1}, J_{\varepsilon} u\right] J_{\varepsilon} \eta_{x}, D^{s-1} J_{\varepsilon} \eta\right)_{L^{2}}+\left(J_{\varepsilon} u D^{s-1} J_{\varepsilon} \eta_{x}, D^{s-1} J_{\varepsilon} \eta\right)_{L^{2}}\right| \\
& \lesssim\left(\left\|u_{x}\right\|_{L^{\infty}}+\left\|\eta_{x}\right\|_{L^{\infty}}\right)\left(\|u\|_{H^{s}}^{2}+\|\eta\|_{H^{s-1}}^{2}\right) .
\end{aligned}
$$

By (A.5), (A.6) and the fact that $D^{s-2}=D^{s} D^{-2}$, we obtain

$$
\begin{aligned}
E_{3} & +E_{4} \\
= & \left(D^{s-2} J_{\varepsilon} \sum_{k=1}^{\infty} \mathcal{L}_{\xi_{k}}^{2} D^{2} J_{\varepsilon} u, D^{s-2} J_{\varepsilon} D^{2} J_{\varepsilon} u\right)_{L^{2}} \\
& +\sum_{k=1}^{\infty}\left(D^{s-2} J_{\varepsilon} \mathcal{L}_{\xi_{k}} D^{2} J_{\varepsilon} u, D^{s-2} J_{\varepsilon} \mathcal{L}_{\xi_{k}} D^{2} J_{\varepsilon} u\right)_{L^{2}} .
\end{aligned}
$$

Since $D^{s-2} J_{\varepsilon} \in \operatorname{OPS}_{1,0}^{s-2}$ (cf. Lemma A.1), we apply Lemma A.5 with $\mathcal{P}=D^{s-2} J_{\varepsilon}$ to arrive at

$$
E_{3}+E_{4} \lesssim\left\|D^{2} J_{\varepsilon} u\right\|_{H^{s-2}}^{2} \leq C\|u\|_{H^{s}}^{2},
$$


where we have used (A.7) in the last inequality. Similarly,

$$
\begin{aligned}
E_{5} & +E_{6} \\
= & \left(D^{s-1} J_{\varepsilon} \sum_{k=1}^{\infty} \mathcal{L}_{\xi_{k}}^{2} J_{\varepsilon} \eta, D^{s-1} J_{\varepsilon} J_{\varepsilon} \eta\right)_{L^{2}} \\
& +\sum_{k=1}^{\infty}\left(D^{s-1} J_{\varepsilon} \mathcal{L}_{\xi_{k}} J_{\varepsilon} \eta, D^{s-1} J_{\varepsilon} \mathcal{L}_{\xi_{k}} J_{\varepsilon} \eta\right)_{L^{2}} \\
\leq & C\left\|J_{\varepsilon} \eta\right\|_{H^{s-1}}^{2} \leq C\|\eta\|_{H^{s-1}}^{2} .
\end{aligned}
$$

Combining the above estimates, we arrive at

$$
\begin{aligned}
2\left(g_{\varepsilon}(X), X\right)_{\mathcal{X}^{s}}+\left\|h_{\varepsilon}(X)\right\|_{\mathcal{L}_{2}\left(\mathbb{U} ; \mathcal{X}^{s}\right)}^{2} & \lesssim\left(1+\left\|u_{X}\right\|_{L^{\infty}}+\left\|\eta_{x}\right\|_{L^{\infty}}\right)\left(\|u\|_{H^{s}}^{2}+\|\eta\|_{H^{s-1}}^{2}\right) \\
& \leq f(\|X\| \mathcal{V})\|X\|_{\mathcal{X}^{s}}^{2},
\end{aligned}
$$

which implies (2.13) with $k(t) \equiv 1$.

Checking $\left(\mathbf{B}_{1}\right)$ in Assumption (B): It is clear that $\mathcal{X}=\mathcal{X}^{s}$ is dense in $\mathcal{Z}=$ $\mathcal{X}^{s-2}$. Since $s-2>\frac{5}{2}$, inequality (2.14) follows directly from Lemma 3.1. Applying Lemma 3.3 yields (2.15).

Checking $\left(\mathbf{B}_{2}\right)$ in Assumption (B): Recall that $\tilde{J}_{\varepsilon}=\left(1-\varepsilon^{2} \Delta\right)^{-1}$ is given in (A.2). Due to (A.7) and $T_{\varepsilon}=Q_{\varepsilon}=\tilde{J}_{\varepsilon},\left(\mathbf{B}_{2}\right)$ is a direct consequence of Assumption (C), which will be checked below.

Checking Assumption (C): It is easy to prove (2.16) and we omit the details here. Then, we notice that

$$
\begin{aligned}
2\left(T_{\varepsilon} g(X), T_{\varepsilon} X\right)_{\mathcal{X}^{s}}+\left\|T_{\varepsilon} h(X)\right\|_{\mathcal{L}_{2}\left(\mathbb{U} ; \mathcal{X}^{s}\right)}^{2} & -2\left(T_{\varepsilon}\left[u u_{x}\right], T_{\varepsilon} u\right)_{H^{s}}-2\left(T_{\varepsilon}\left[u \eta_{x}\right], T_{\varepsilon} \eta\right)_{H^{s-1}} \\
& +\left(D^{s} T_{\varepsilon} D^{-2} \sum_{k=1}^{\infty} \mathcal{L}_{\xi_{k}}^{2} D^{2} u, D^{s} T_{\varepsilon} u\right)_{L^{2}} \\
& +\sum_{k=1}^{\infty}\left(D^{s} T_{\varepsilon} D^{-2} \mathcal{L}_{\xi_{k}} D^{2} u, D^{s} T_{\varepsilon} D^{-2} \mathcal{L}_{\xi_{k}} D^{2} u\right)_{L^{2}} \\
& +\left(D^{s-1} T_{\varepsilon} \sum_{k=1}^{\infty} \mathcal{L}_{\xi_{k}}^{2} \eta, D^{s-1} T_{\varepsilon} \eta\right)_{L^{2}} \\
& +\sum_{k=1}^{\infty}\left(D^{s-1} T_{\varepsilon} \mathcal{L}_{\xi_{k}} \eta, D^{s-1} T_{\varepsilon} \mathcal{L}_{\xi_{k}} \eta\right)_{L^{2}}=\sum_{i=1}^{6} R_{i} .
\end{aligned}
$$

For the first term, we have that 


$$
\begin{aligned}
\left|R_{1}\right| & =2\left|\left(D^{s} T_{\varepsilon}\left[u u_{x}\right], D^{s} T_{\varepsilon} u\right)_{L^{2}}\right| \\
& \leq 2\left|\left(\left[D^{s}, u\right] u_{x}, D^{s} T_{\varepsilon}^{2} u\right)_{L^{2}}+\left(\left[T_{\varepsilon}, u\right] D^{s} u_{x}, D^{s} T_{\varepsilon} u\right)_{L^{2}}+\left(u D^{s} T_{\varepsilon} u_{x}, D^{s} T_{\varepsilon} u\right)_{L^{2}}\right| \\
& \leq C\left\|u_{x}\right\|_{L^{\infty}}\|u\|_{H^{s}}\left\|T_{\varepsilon} u\right\|_{H^{s}}+C\left\|u_{x}\right\|_{L^{\infty}}\left\|T_{\varepsilon} u\right\|_{H^{s}}^{2},
\end{aligned}
$$

where we have used Lemmas A.3 and A.4, integration by parts, embedding $H^{s-1} \hookrightarrow$ $W^{1, \infty},($ A.6) and (A.7). Similarly, we can show that

$$
\begin{aligned}
& \left|R_{2}\right|=2\left|\left(D^{s-1} T_{\varepsilon}\left[u \eta_{x}\right], D^{s-1} T_{\varepsilon} \eta\right)_{L^{2}}\right| \\
& =2 \mid\left(\left[D^{s-1}, u\right] \eta_{x}, D^{s-1} T_{\varepsilon}^{2} \eta\right)_{L^{2}} \\
& +\left(\left[T_{\varepsilon}, u\right] D^{s-1} \eta_{x}, D^{s-1} T_{\varepsilon} \eta\right)_{L^{2}}+\left(u D^{s-1} T_{\varepsilon} \eta_{x}, D^{s-1} T_{\varepsilon} \eta\right)_{L^{2}} \mid \\
& \leq C\left(\left\|u_{x}\right\|_{L^{\infty}}\|\eta\|_{H^{s-1}}\left\|T_{\varepsilon} \eta\right\|_{H^{s-1}}+\left\|\eta_{x}\right\|_{L^{\infty}}\|u\|_{H^{s}}\left\|T_{\varepsilon} \eta\right\|_{H^{s-1}}\right)+C\left\|u_{x}\right\|_{L^{\infty}}\left\|T_{\varepsilon} \eta\right\|_{H^{s}}^{2} \\
& \lesssim\left\|u_{x}\right\|_{L^{\infty}}\|\eta\|_{H^{s-1}}\left\|T_{\varepsilon} \eta\right\|_{H^{s-1}}+\left\|\eta_{x}\right\|_{L^{\infty}}\|u\|_{H^{s}}\left\|T_{\varepsilon} \eta\right\|_{H^{s-1}} \text {. }
\end{aligned}
$$

Using Lemma A.5 yields

$$
\begin{aligned}
& R_{3}+R_{4}=\left(D^{s-2} T_{\varepsilon} \sum_{k=1}^{\infty} \mathcal{L}_{\xi_{k}}^{2} D^{2} u, D^{s-2} T_{\varepsilon} D^{2} u\right)_{L^{2}} \\
&+\sum_{k=1}^{\infty}\left(-D^{s-2} T_{\varepsilon} \mathcal{L}_{\xi_{k}} D^{2} u,-D^{s-2} T_{\varepsilon} \mathcal{L}_{\xi_{k}} D^{2} u\right)_{L^{2}} \\
& \lesssim\left\|D^{2} u\right\|_{H^{s-2}}^{2} \leq\|u\|_{H^{s}}^{2} .
\end{aligned}
$$

and analogously

$$
\begin{aligned}
R_{5}+R_{6}= & \left(D^{s-1} T_{\varepsilon} \sum_{k=1}^{\infty} \mathcal{L}_{\xi_{k}}^{2} \eta, D^{s-1} T_{\varepsilon} \eta\right)_{L^{2}} \\
& +\sum_{k=1}^{\infty}\left(D^{s-1} T_{\varepsilon} \mathcal{L}_{\xi_{k}} \eta, D^{s-1} T_{\varepsilon} \mathcal{L}_{\xi_{k}} \eta\right)_{L^{2}} \lesssim\|\eta\|_{H^{s-1}}^{2}
\end{aligned}
$$

Gathering together the above estimates and noticing (A.7), we get

$$
\begin{aligned}
& 2\left(T_{\varepsilon} g(X), T_{\varepsilon} X\right)_{\mathcal{X}^{s}}+\left\|T_{\varepsilon} h(X)\right\|_{\mathcal{L}_{2}\left(\mathbb{U} ; \mathcal{X}^{s}\right)}^{2} \\
& \quad \lesssim\left(1+\left\|u_{X}\right\|_{L^{\infty}}+\left\|\eta_{x}\right\|_{L^{\infty}}\right)\left(\|u\|_{H^{s}}^{2}+\|\eta\|_{H^{s-1}}^{2}\right) \leq f(\|X\| \mathcal{V})\|X\|_{\mathcal{X}^{s}}^{2},
\end{aligned}
$$

which gives (2.20). We are just left to show (2.19) to conclude the proof of Theorem 3.1. To this end, we recall (3.16) and consider 


$$
\begin{aligned}
-\left(T_{\varepsilon} h_{k}(X), T_{\varepsilon} X\right)_{\mathcal{X}^{s}}= & \left(T_{\varepsilon} D^{s-2} \mathcal{L}_{\xi_{k}} D^{2} u, T_{\varepsilon} D^{s} u\right)_{L^{2}} \\
& +\left(T_{\varepsilon} D^{s-1} \mathcal{L}_{\xi_{k}} \eta, T_{\varepsilon} D^{s-1} \eta\right)_{L^{2}} \\
= & \left(\mathcal{P}_{1} \mathcal{L}_{\xi_{k}} D^{2} u, \mathcal{P}_{1} D^{2} u\right)_{L^{2}}+\left(\mathcal{P}_{2} \mathcal{L}_{\xi_{k}} \eta, \mathcal{P}_{2} \eta\right)_{L^{2}} \\
= & \left(\mathcal{T}_{1} D^{2} u, \mathcal{P}_{1} D^{2} u\right)_{L^{2}}+\left(\mathcal{L}_{\xi_{k}} \mathcal{P}_{1} D^{2} u, \mathcal{P}_{1} D^{2} u\right)_{L^{2}} \\
& +\left(\mathcal{T}_{2} \eta, \mathcal{P}_{2} \eta\right)_{L^{2}}+\left(\mathcal{L}_{\xi_{k}} \mathcal{P}_{2} \eta, \mathcal{P}_{2} \eta\right)_{L^{2}} \\
& :=\sum_{i=1}^{4} J_{i},
\end{aligned}
$$

where $\mathcal{P}_{1}:=T_{\varepsilon} D^{s-2} \in \operatorname{OPS}_{1,0}^{s-2}, \mathcal{P}_{2}:=T_{\varepsilon} D^{s-1} \in \operatorname{OPS}_{1,0}^{s-1}$ (cf. Lemma A.1), and $\mathcal{T}_{1}=\left[\mathcal{P}_{1}, \mathcal{L}_{\xi_{k}}\right], \mathcal{T}_{2}=\left[\mathcal{P}_{2}, \mathcal{L}_{\xi_{k}}\right]$. Using integration by parts, (3.5) and (A.5), we have that

$$
\left|J_{2}\right|+\left|J_{4}\right| \lesssim\left\|\partial_{x} \xi_{k}\right\|_{L^{\infty}}\left(\left\|\mathcal{P}_{1} D^{2} u\right\|_{L^{2}}^{2}+\left\|\mathcal{P}_{2} \eta\right\|_{L^{2}}^{2}\right) \lesssim\left\|\partial_{x} \xi_{k}\right\|_{L^{\infty}}\left\|T_{\varepsilon} X\right\|_{\mathcal{X}^{s}}^{2}
$$

Using (A.6) and (A.5), we have

$$
\begin{aligned}
J_{3}= & \left(\mathcal{T}_{2} \eta, \mathcal{P}_{2} \eta\right)_{L^{2}} \\
= & \left(D^{s-1} \mathcal{L}_{\xi_{k}} \eta, D^{s-1} T_{\varepsilon}^{2} \eta\right)_{L^{2}}-\left(\mathcal{L}_{\xi_{k}} D^{s-1} T_{\varepsilon} \eta, D^{s-1} T_{\varepsilon} \eta\right)_{L^{2}} \\
= & \left(D^{s-1} \xi_{k} \partial_{x} \eta, D^{s-1} T_{\varepsilon}^{2} \eta\right)_{L^{2}}+\left(D^{s-1} \eta \partial_{x} \xi_{k}, D^{s-1} T_{\varepsilon}^{2} \eta\right)_{L^{2}} \\
& -\left(\mathcal{L}_{\xi_{k}} D^{s-1} T_{\varepsilon} \eta, D^{s-1} T_{\varepsilon} \eta\right)_{L^{2}} \\
= & \left(\left[D^{s-1}, \xi_{k}\right] \partial_{x} \eta, D^{s-1} T_{\varepsilon}^{2} \eta\right)_{L^{2}}+\left(T_{\varepsilon} \xi_{k} D^{s-1} \partial_{x} \eta, D^{s-1} T_{\varepsilon} \eta\right)_{L^{2}} \\
& +\left(D^{s-1} \eta \partial_{x} \xi_{k}, D^{s-1} T_{\varepsilon}^{2} \eta\right)_{L^{2}}-\left(\mathcal{L}_{\xi_{k}} D^{s-1} T_{\varepsilon} \eta, D^{s-1} T_{\varepsilon} \eta\right)_{L^{2}} \\
:= & \sum_{i=1}^{4} K_{i} .
\end{aligned}
$$

On account of $H^{s-1} \hookrightarrow W^{1, \infty}$ and integration by parts, it holds that

$$
\left|K_{3}\right| \lesssim\left\|\eta \partial_{x} \xi_{k}\right\|_{H^{s-1}}\left\|T_{\varepsilon} \eta\right\|_{H^{s-1}} \leq\left\|\xi_{k}\right\|_{H^{s}}\|\eta\|_{H^{s-1}}\left\|T_{\varepsilon} \eta\right\|_{H^{s-1}}
$$

and

$$
\left|K_{4}\right| \lesssim\left\|\partial_{x} \xi_{k}\right\|_{L^{\infty}}\left\|T_{\varepsilon} \eta\right\|_{H^{s-1}}^{2} \lesssim\left\|\partial_{x} \xi_{k}\right\|_{L^{\infty}}\|\eta\|_{H^{s-1}}\left\|T_{\varepsilon} \eta\right\|_{H^{s-1}}
$$


Then, we apply Lemma A.4 to $K_{1}$ to find

$$
\left|K_{1}\right| \lesssim\left\|\xi_{k}\right\|_{H^{s}}\|\eta\|_{H^{s-1}}\left\|T_{\varepsilon} \eta\right\|_{H^{s-1}}
$$

For $K_{2}$, we use Lemma A.3 and integration by parts to derive

$$
\begin{aligned}
\left|K_{2}\right| & \lesssim\left|\left(\left[T_{\varepsilon}, \xi_{k}\right] \partial_{x} D^{s-1} \eta, D^{s-1} T_{\varepsilon} \eta\right)\right|+\left|\left(\xi_{k} \partial_{x} D^{s-1} T_{\varepsilon} \eta, D^{s-1} T_{\varepsilon} \eta\right)\right| \\
& \lesssim\left\|\partial_{x} \xi_{k}\right\|_{L^{\infty}}\|\eta\|_{H^{s-1}}\left\|T_{\varepsilon} \eta\right\|_{H^{s-1}}
\end{aligned}
$$

Therefore,

$$
\left|J_{3}\right|=\left|\left(\mathcal{T}_{2} \eta, \mathcal{P}_{2} \eta\right)_{L^{2}}\right| \lesssim\left\|\xi_{k}\right\|_{H^{s}}\|\eta\|_{H^{s-1}}\left\|T_{\varepsilon} \eta\right\|_{H^{s-1}} .
$$

The form $J_{4}=\left(\mathcal{T}_{1} D^{2} u, \mathcal{P}_{1} D^{2} u\right)_{L^{2}}$ can be handled in the same way using $H^{s-2} \hookrightarrow$ $W^{1, \infty}$. Hence, we have

$$
\left|J_{3}\right|=\left|\left(\mathcal{T}_{1} f, \mathcal{P}_{1} f\right)_{L^{2}}\right| \lesssim\left\|\xi_{k}\right\|_{H^{s}}\|f\|_{H^{s-2}}\left\|T_{\varepsilon} f\right\|_{H^{s-2}} \lesssim\left\|\xi_{k}\right\|_{H^{s}}\|u\|_{H^{s}}\left\|T_{\varepsilon} u\right\|_{H^{s}}
$$

Now we summarize the above estimates, and use (3.17) and Assumption (D) to arrive at

$$
\sum_{k=1}^{\infty}\left|\left(T_{\varepsilon} h(X) e_{k}, T_{\varepsilon} X\right) \mathcal{X}^{s}\right|^{2} \lesssim \sum_{k=1}^{\infty}\left\|\xi_{k}\right\|_{H^{s}}^{2}\|X\|_{\mathcal{X}^{s}}^{2}\left\|T_{\varepsilon} X\right\|_{\mathcal{X}^{s}}^{2} \leq C\|X\|_{\mathcal{X}^{s}}^{2}\left\|T_{\varepsilon} X\right\|_{\mathcal{X}^{s}}^{2}
$$

Hence, we obtain inequality (2.19) and complete the proof.

\subsection{The Stochastic CCF Model: Proof of Theorem 3.2}

In this section we will apply Theorem 2.1 to (3.11) with $x \in \mathbb{T}$ to obtain Theorem 3.2. To that purpose, we set $X=\theta$ and

$$
\left\{\begin{aligned}
b(t, X) & =b(X)=0 \\
g(t, X) & =g(X)=-(\mathcal{H} \theta) \partial_{x} \theta+\frac{1}{2} \sum_{k=1}^{\infty} \mathcal{L}_{\xi_{k}}^{2} \theta \\
h^{k}(t, X) & =h^{k}(X)=-\mathcal{L}_{\xi_{k}} \theta, \quad k \in \mathbb{N} .
\end{aligned}\right.
$$

As in (3.17), for a fixed separable Hilbert space $\mathbb{U}$ with a complete orthonormal basis $\left\{e_{i}\right\}_{i \in \mathbb{N}}$, we define $h(X) \in \mathcal{L}\left(\mathbb{U} ; H^{s}\right)$ such that

$$
h(X)\left(e_{k}\right)=h^{k}(X), \quad k \in \mathbb{N} .
$$


With the above notations, we reformulate (3.11) in the abstract form, i.e.,

$$
\left\{\begin{array}{l}
\mathrm{d} X=(b(X)+g(X)) \mathrm{d} t+h(X) \mathrm{d} \mathcal{W} \\
X(0)=\theta_{0}
\end{array}\right.
$$

To prove Theorem 3.2, we would like to invoke Theorem 2.1 to this setting. To do that, we just need to check the Assumptions (A), (B) and (C). Now we let $r \in$ $(3 / 2, s-2)$, and then, let

$$
\mathcal{X}^{s}=H^{s} \text { and } \mathcal{V}=H^{r}
$$

\subsubsection{Estimates on Nonlinear Terms}

Analogously to Sect. 3.3.1 we will need the following auxiliary lemmas.

Lemma 3.4 Let Assumption (D) hold true and $s>5 / 2$. If $X=\theta \in \mathcal{X}^{s}$, then $g$ : $\mathcal{X}^{s} \rightarrow \mathcal{X}^{s-2}$ and $h: \mathcal{X}^{s} \rightarrow \mathcal{L}_{2}\left(\mathbb{U} ; \mathcal{X}^{s-1}\right)$ such that

$$
\|g(X)\|_{\mathcal{X}^{s-2}} \lesssim 1+\|X\|_{\mathcal{X}^{s}}^{2}
$$

and

$$
\|h(X)\|_{\mathcal{L}_{2}\left(\mathbb{U} ; \mathcal{X}^{s-1}\right)} \lesssim\|X\| \mathcal{X}^{s}
$$

Proof Using $H^{s-2} \hookrightarrow W^{1, \infty}$, the continuity of the Hilbert transform for $s \geq 0$ and Remark 3.1, one can prove the above estimates directly. We omit the details for exposition clearness.

Lemma 3.5 Let $X=\theta \in \mathcal{X}^{s}$ and $Y=\rho \in \mathcal{X}^{s}$. Then, we have that for $s>7 / 2$,

$$
\begin{aligned}
& 2(g(t, X)-g(t, Y), X-Y) \mathcal{X}_{\mathcal{X}^{s-2}}+\|h(t, X)-h(t, Y)\|_{\mathcal{L}_{2}\left(\mathbb{U} ; \mathcal{X}^{s-2}\right)}^{2} \\
& \quad \lesssim\left(1+\|X\|_{\mathcal{X}^{s}}^{2}+\|Y\|_{\mathcal{X}^{s}}^{2}\right)\|X-Y\|_{\mathcal{X}^{s-2}}^{2} .
\end{aligned}
$$

Proof Recalling (3.25) and (3.26), we have

$$
\begin{aligned}
& 2(g(X)-g(Y), X-Y) \mathcal{X}^{s-2}+\|h(t, X)-h(t, Y)\|_{\mathcal{L}_{2}\left(\mathbb{U} ; \mathcal{X}^{s-2}\right)}^{2} \\
& \quad=2\left((\mathcal{H} \rho) \rho_{x}-(\mathcal{H} \theta) \theta_{x}, \theta-\rho\right)_{H^{s-2}}+\left(\sum_{k=1}^{\infty} \mathcal{L}_{\xi_{k}}^{2}(\theta-\rho), \theta-\rho\right)_{H^{s-2}} \\
& \quad+\sum_{k=1}^{\infty}\left(\mathcal{L}_{\xi_{k}}(\theta-\rho), \mathcal{L}_{\xi_{k}}(\theta-\rho)\right)_{H^{s-2}} .
\end{aligned}
$$


Because $H^{s-2} \hookrightarrow W^{1, \infty}$, we use Remark 3.1, Lemma A.4, the continuity of the Hilbert transform and integration by parts to bound the first term as

$$
\begin{aligned}
& \left((\mathcal{H} \rho) \rho_{x}-(\mathcal{H} \theta) \theta_{x}, \theta-\rho\right)_{H^{s-2}} \\
& \quad \lesssim\left|\left(D^{s-2}(\mathcal{H} \rho)(\theta-\rho)_{x}, D^{s-2}(\theta-\rho)\right)_{L^{2}}\right|+\left|\left(D^{s-2}(\mathcal{H}(\theta-\rho)) \theta_{x}, D^{s-2}(\theta-\rho)\right)_{L^{2}}\right| \\
& \quad \lesssim\left\|\left[D^{s-2}, \mathcal{H} \rho\right](\theta-\rho)_{x}\right\|_{L^{2}}\|\theta-\rho\|_{H^{s-2}}+\left\|\partial_{x} \mathcal{H} \rho\right\|_{L^{\infty}}\|\theta-\rho\|_{H^{s-2}}^{2} \\
& \quad+\left\|\left[D^{s-2}, \mathcal{H}(\theta-\rho)\right] \theta_{x}\right\|_{L^{2}}\|\theta-\rho\|_{H^{s-2}}+\left\|\partial_{x} \mathcal{H}(\theta-\rho)\right\|_{L^{\infty}}\|\theta-\rho\|_{H^{s-2}}^{2} \\
& \quad \lesssim\left(\|\rho\|_{H^{s}}+\|\theta\|_{H^{s}}\right)\|\theta-\rho\|_{H^{s-2}}^{2} .
\end{aligned}
$$

The last two terms can be bounded by invoking Lemma A.5

to obtain

$$
\begin{aligned}
& \left(D^{s-2} \sum_{k=1}^{\infty} \mathcal{L}_{\xi_{k}}^{2}(\theta-\rho), D^{s-2}(\theta-\rho)\right)_{L^{2}} \\
& +\sum_{k=1}^{\infty}\left(D^{s-2} \mathcal{L}_{\xi_{k}}(\theta-\rho), D^{s-2} \mathcal{L}_{\xi_{k}}(\theta-\rho)\right)_{L^{2}} \lesssim\|\theta-\rho\|_{H^{s-2}}^{2}
\end{aligned}
$$

Collecting the above estimates, we obtain the desired result.

\subsubsection{Proof of Theorem 3.2}

To avoid unnecessary repetition, we just sketch the main points of the proof since it is similar to the proof of Theorem 3.1. Recalling (A.1), we define

$$
g_{\varepsilon}(X)=-J_{\varepsilon}\left[\left(\mathcal{H} J_{\varepsilon} \theta\right) \partial_{x} J_{\varepsilon} \theta\right]+\frac{1}{2} J_{\varepsilon}^{3} \sum_{k=1}^{\infty} \mathcal{L}_{\xi_{k}}^{2} J_{\varepsilon} \theta
$$

Let

$$
h_{\varepsilon}^{k}(X)=-J_{\varepsilon} \mathcal{L}_{\xi_{k}} J_{\varepsilon} \theta
$$

Similar to (3.17), we define $h_{\varepsilon}(X) \in \mathcal{L}\left(\mathbb{U} ; \mathcal{X}^{s}\right)$ such that

$$
h_{\varepsilon}(X)\left(e_{k}\right)=h_{\varepsilon}^{k}(X), \quad k \in \mathbb{N}
$$

We now prove that all the requirements in Assumptions (A), (B) and (C) hold true for the following choice:

$-\mathcal{X}:=\mathcal{X}^{s}, \mathcal{Y}:=\mathcal{X}^{s-1}$ and $\mathcal{Z}:=\mathcal{X}^{s-2}$, where $\mathcal{X}^{s}$ is given in (3.28), and $\mathcal{V}:=H^{r}$ with $r \in(3 / 2, s-2)$;

$-b, g, h, g_{\varepsilon}$ and $h_{\varepsilon}$ are given in (3.25), (3.29), (3.30) and (3.31), respectively; 
$-k(\cdot) \equiv 1, f(\cdot)=C(1+\cdot), q(\cdot)=C\left(1+\cdot^{5}\right)$ for some $C>1$ large enough;

$-T_{\varepsilon}=Q_{\varepsilon}=\tilde{J}_{\varepsilon}$, where $\tilde{J}_{\varepsilon}$ is given in (A.2).

Let $s>7 / 2$. Obviously, $\mathcal{X} \hookrightarrow \mathcal{Y} \hookrightarrow \hookrightarrow \mathcal{Z} \hookrightarrow \mathcal{V}$. Moreover, Lemma 3.4 implies $g: \mathcal{X}^{s} \rightarrow \mathcal{X}^{s-2}$ and $h: \mathcal{X}^{s} \rightarrow \mathcal{L}_{2}\left(\mathbb{U} ; \mathcal{X}^{s-1}\right)$. Hence, the stochastic integral in (3.27) is a well defined $\mathcal{X}^{s-1}$-valued local martingale. It is easy to check that $g$ and $h$ are continuous in $X \in \mathcal{X}^{s}$.

Checking $\left(\mathbf{A}_{1}\right)$ in Assumption (A): Trivial, since $b(t, X) \equiv 0$.

Checking ( $\left.\mathbf{A}_{2}\right)$ in Assumption (A): By the construction of $g_{\varepsilon}(X)$ and $h_{\varepsilon}(X),(\mathrm{A} .4)$, Lemma 3.4 and Assumption (D), ( $\left.\mathbf{A}_{2}\right)$ is verified.

Checking $\left(\mathbf{A}_{3}\right)$ in Assumption (A): Since (3.30) enjoys similar estimates as we established for (3.22), the first part (2.12) can be proved as before. Therefore, we just need to show (2.13). For all $X=\theta \in \mathcal{X}^{s}$, we have

$$
\begin{aligned}
2\left(g_{\varepsilon}(X), X\right)_{\mathcal{X}^{s}}+\left\|h_{\varepsilon}(X)\right\|_{\mathcal{L}_{2}\left(\mathbb{U} ; \mathcal{X}^{s}\right)}^{2}= & -2\left(D^{s} J_{\varepsilon}\left[\mathcal{H} J_{\varepsilon} \theta \partial_{x} J_{\varepsilon} \theta\right], D^{s} \theta\right)_{L^{2}} \\
& +\left(D^{s} J_{\varepsilon}^{3} \sum_{k=1}^{\infty} \mathcal{L}_{\xi_{k}}^{2} J_{\varepsilon} \theta, D^{s} \theta\right)_{L^{2}} \\
& +\sum_{k=1}^{\infty}\left(D^{s} J_{\varepsilon} \mathcal{L}_{\xi_{k}} J_{\varepsilon} \theta, D^{s} J_{\varepsilon} \mathcal{L}_{\xi_{k}} J_{\varepsilon} \theta\right)_{L^{2}} \\
:= & \sum_{i=1}^{3} E_{i} .
\end{aligned}
$$

Invoking Lemma A.5 with $\mathcal{P}=D^{s} J_{\varepsilon} \in \mathrm{OPS}_{1,0}^{s}$ (cf. Lemma A.1), we have that

$$
\begin{aligned}
E_{2}+E_{3}= & \left(D^{s} J_{\varepsilon} \sum_{k=1}^{\infty} \mathcal{L}_{\xi_{k}}^{2} J_{\varepsilon} \theta, D^{s} J_{\varepsilon} J_{\varepsilon} \theta\right)_{L^{2}} \\
& +\sum_{k=1}^{\infty}\left(D^{s} J_{\varepsilon} \mathcal{L}_{\xi_{k}} J_{\varepsilon} \theta, D^{s} J_{\varepsilon} \mathcal{L}_{\xi_{k}} J_{\varepsilon} \theta\right)_{L^{2}} \\
\leq & C\left\|J_{\varepsilon} \theta\right\|_{H^{s}}^{2} \leq C\|\theta\|_{H^{s}}^{2} .
\end{aligned}
$$

To bound the first term, we notice that $H^{r} \hookrightarrow W^{1, \infty}$; then, we use Lemma A.4, integration by parts, (A.7) and (A.8) to find

$$
\begin{aligned}
\left|E_{1}\right| & =2\left|\left(\mathcal{H} J_{\varepsilon} \theta \partial_{x} J_{\varepsilon} D^{s} \theta, D^{s} J_{\varepsilon} \theta\right)_{L^{2}}+2\left(\left[D^{s}, \mathcal{H} J_{\varepsilon} \theta\right] \partial_{x} J_{\varepsilon} \theta, D^{s} J_{\varepsilon} \theta\right)_{L^{2}}\right| \\
& \lesssim\left\|\mathcal{H} \partial_{x} \theta\right\|_{L^{\infty}}\left\|D^{s} J_{\varepsilon} \theta\right\|_{L^{2}}^{2}+\left\|\left[D^{s}, \mathcal{H} J_{\varepsilon} \theta\right] \partial_{x} J_{\varepsilon} \theta\right\|_{L^{2}}\left\|D^{s} J_{\varepsilon} \theta\right\|_{L^{2}} \\
\lesssim & \left\|\mathcal{H} \partial_{x} J_{\varepsilon} \theta\right\|_{L^{\infty}}\left\|D^{s} J_{\varepsilon} \theta\right\|_{L^{2}}^{2} \\
& +\left(\left\|\partial_{x} \mathcal{H} J_{\varepsilon} \theta\right\|_{L^{\infty}}\left\|D^{s-1} \partial_{x} J_{\varepsilon} \theta\right\|_{L^{2}}\right. \\
& \left.+\left\|D^{s} \mathcal{H} J_{\varepsilon} \theta\right\|_{L^{2}}\left\|\partial_{x} J_{\varepsilon} \theta\right\|_{L^{\infty}}\right)\left\|D^{s} J_{\varepsilon} \theta\right\|_{L^{2}} \\
& \lesssim\left\|\mathcal{H} \partial_{x} J_{\varepsilon} \theta\right\|_{L^{\infty}}\|\theta\|_{H^{s}}^{2}
\end{aligned}
$$




$$
\begin{aligned}
&+\left\|\partial_{x} \mathcal{H} J_{\varepsilon} \theta\right\|_{L^{\infty}}\|\theta\|_{H^{s}}^{2}+\left\|\partial_{x} J_{\varepsilon} \theta\right\|_{L^{\infty}}\|\theta\|_{H^{s}}^{2} \\
& \lesssim\left\|\mathcal{H} \partial_{x} \theta\right\|_{L^{\infty}}\|\theta\|_{H^{s}}^{2}+\left\|\partial_{x} \theta\right\|_{L^{\infty}}\|\theta\|_{H^{s}}^{2}
\end{aligned}
$$

Combining the above estimates, we arrive at

$$
\begin{aligned}
2\left(g_{\varepsilon}(X), X\right)_{\mathcal{X}^{s}}+\left\|h_{\varepsilon}(t, X)\right\|_{\mathcal{L}_{2}\left(\mathbb{U} ; \mathcal{X}^{s}\right)}^{2} & \lesssim\left(\left\|\mathcal{H} \partial_{x} \theta\right\|_{L^{\infty}}+\left\|\partial_{x} \theta\right\|_{L^{\infty}}\right)\|\theta\|_{H^{s}}^{2} \\
& \leq f(\|X\| \mathcal{V})\|X\|_{\mathcal{X}^{s}}^{2}
\end{aligned}
$$

which implies (2.13).

Checking $\left(\mathbf{B}_{1}\right)$ in Assumption (B): The dense embedding $\mathcal{X}=\mathcal{X}^{s} \hookrightarrow \mathcal{Z}=\mathcal{X}^{s-2}$ and (2.14) is clear. Applying Lemma 3.5, we infer (2.15).

Checking $\left(\mathbf{B}_{2}\right)$ in Assumption (B): As before, this is a direct consequence of Assumption (C), which will be shown next.

Checking Assumption (C): Following the same way as we proved (3.24), we have that for some $C>1$,

$$
\sum_{k=1}^{\infty}\left|\left(T_{\varepsilon} h(\theta) e_{k}, T_{\varepsilon} \theta\right)_{H^{s}}\right|^{2} \leq C\|\theta\|_{H^{s}}^{2}\left\|T_{\varepsilon} \theta\right\|_{H^{s}}^{2}
$$

Hence, (2.19) holds. Now we just need to prove (2.20). Indeed,

$$
\begin{aligned}
& 2\left(T_{\varepsilon} g(X), T_{\varepsilon} X\right)_{\mathcal{X}^{s}}+\left\|T_{\varepsilon} h(X)\right\|_{\mathcal{L}_{2}\left(\mathbb{U} ; \mathcal{X}^{s}\right)}^{2} \\
& =-2\left(T_{\varepsilon}\left[\mathcal{H} \theta \theta_{x}\right], T_{\varepsilon} \theta\right)_{H^{s}}+\left(D^{s} T_{\varepsilon} \sum_{k=1}^{\infty} \mathcal{L}_{\xi_{k}}^{2} \theta, D^{s} T_{\varepsilon} \theta\right)_{L^{2}} \\
& \quad+\sum_{k=1}^{\infty}\left(D^{s} T_{\varepsilon} \mathcal{L}_{\xi_{k}} \theta, D^{s} T_{\varepsilon} \mathcal{L}_{\xi_{k}} \theta\right)_{L^{2}} \\
& \quad:=\sum_{i=1}^{3} R_{i} .
\end{aligned}
$$

Using Lemma A.4, (A.8), (A.9), integration by parts, Lemma A.3 and (A.7), we have

$$
\begin{aligned}
\left|R_{1}\right| \leq & 2 \mid\left(\left[D^{s}, \mathcal{H} \theta\right] \theta_{x}, D^{s} T_{\varepsilon}^{2} \theta\right)_{L^{2}}+\left(\left[T_{\varepsilon}, \mathcal{H} \theta\right] D^{s} \theta_{x}, D^{s} T_{\varepsilon} \theta\right)_{L^{2}} \\
& +\left(\mathcal{H} \theta D^{s} T_{\varepsilon} \theta_{x}, D^{s} T_{\varepsilon} \theta\right)_{L^{2}} \mid \\
\leq & C\left\|\theta_{x}\right\|_{L^{\infty}}\|\theta\|_{H^{s}}^{2}+C\left\|\mathcal{H} \theta_{x}\right\|_{L^{\infty}}\|\theta\|_{H^{s}}^{2} \lesssim\left(\left\|\theta_{x}\right\|_{L^{\infty}}+\left\|\mathcal{H} \theta_{x}\right\|_{L^{\infty}}\right)\|\theta\|_{H^{s}}^{2}
\end{aligned}
$$

Using Lemma (A.5) with $\mathcal{P}=D^{s} T_{\varepsilon} \in \mathrm{OPS}_{1,0}^{s}$ (cf. Lemma A.1), we have that

$$
R_{2}+R_{3}=\left(D^{s} T_{\varepsilon} \sum_{k=1}^{\infty} \mathcal{L}_{\xi_{k}}^{2} \theta, D^{s} T_{\varepsilon} \theta\right)_{L^{2}}+\sum_{k=1}^{\infty}\left(D^{s} T_{\varepsilon} \mathcal{L}_{\xi_{k}} \theta, D^{s} T_{\varepsilon} \mathcal{L}_{\xi_{k}} \theta\right)_{L^{2}} \lesssim\|\theta\|_{H^{s}}^{2}
$$


Combining the above estimates, we find some $C>1$ such that,

$$
2\left(T_{\varepsilon} g(X), T_{\varepsilon} X\right)_{\mathcal{X}^{s}}+\left\|T_{\varepsilon} h(X)\right\|_{\mathcal{L}_{2}\left(\mathbb{U} ; \mathcal{X}^{s}\right)}^{2} \leq C\left(1+\left\|\theta_{x}\right\|_{L^{\infty}}+\left\|\mathcal{H} \theta_{x}\right\|_{L^{\infty}}\right)\|\theta\|_{H^{s}}^{2}
$$

Due to $\mathcal{V}=H^{r} \hookrightarrow W^{1, \infty}$ and (A.9), (2.20) holds true. Therefore, we can apply Theorem 2.1 to obtain the existence, uniqueness of pathwise solutions, together with the blow-up criterion

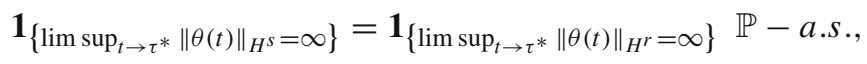

where $r \in(3 / 2, s-2)$ is arbitrary. Now we only need to improve the above blow-up criterion to (3.14). To this end, we proceed as in the proof of (2.22) (cf. (2.49)). For $m, l \in \mathbb{N}$, we define

$$
\sigma_{1, m}=\inf \left\{t \geq 0:\|\theta(t)\|_{H^{s}} \geq m\right\}, \quad \sigma_{2, l}=\inf \left\{t \geq 0:\left\|\theta_{x}(t)\right\|_{L^{\infty}}+\left\|\mathcal{H} \theta_{x}\right\|_{L^{\infty}} \geq l\right\},
$$

where inf $\varnothing=\infty$. Denote $\sigma_{1}=\lim _{m \rightarrow \infty} \sigma_{1, m}$ and $\sigma_{2}=\lim _{l \rightarrow \infty} \sigma_{2, l}$. Now we fix a $r \in(3 / 2, s-2)$. Then,

$$
\left\|\theta_{x}(t)\right\|_{L^{\infty}}+\left\|\mathcal{H} \theta_{x}\right\|_{L^{\infty}} \lesssim\|\theta(t)\|_{H^{r}} \lesssim\|\theta(t)\|_{H^{s}}
$$

From this, it is obvious that $\sigma_{1} \leq \sigma_{2} \mathbb{P}-$ a.s. To prove $\sigma_{1}=\sigma_{2} \mathbb{P}-$ a.s., we need to prove $\sigma_{1} \geq \sigma_{2} \mathbb{P}-$ a.s. In the same way as we prove (2.49), we only need to prove

$$
\mathbb{P}\left\{\sup _{t \in\left[0, \sigma_{2, l} \wedge N\right]}\|\theta(t)\|_{H^{s}}<\infty\right\}=1 \quad \forall N, l \in \mathbb{N} .
$$

It follows from (3.32) and (3.33) that

$$
\begin{aligned}
E & \sup _{t \in\left[0, \sigma_{2, l} \wedge N\right]}\left\|T_{\varepsilon} \theta\right\|_{H^{s}}^{2}-\mathbb{E}\left\|T_{\varepsilon} \theta_{0}\right\|_{H^{s}}^{2} \\
\leq & C \mathbb{E}\left(\int_{0}^{\sigma_{2, l} \wedge N}\|\theta\|_{H^{s}}^{2}\left\|T_{\varepsilon} \theta\right\|_{H^{s}}^{2} \mathrm{~d} t\right)^{\frac{1}{2}}\left(1+\left\|\theta_{x}\right\|_{L^{\infty}}+\left\|\mathcal{H} \theta_{x}\right\|_{L^{\infty}}\right)\|\theta\|_{H^{s}}^{2} \mathrm{~d} t \\
& +C \mathbb{E} \int_{0}^{\sigma_{2, l} \wedge N}\left(\sup _{t \in\left[0, \sigma_{2, l} \wedge N\right]}\left\|T_{\varepsilon} \theta\right\|_{H^{s}}^{2} \int_{0}^{\sigma_{2, l} \wedge N}\|\theta\|_{H^{s}}^{2} \mathrm{~d} t\right)^{\frac{1}{2}}+C_{l} \mathbb{E} \int_{0}^{\sigma_{2, l} \wedge N}\|\theta\|_{H^{s}}^{2} \mathrm{~d} t \\
\leq & C \mathbb{E}\left(\sup _{t \in\left[0, \sigma_{2, l} \wedge N\right]}\left\|T_{\varepsilon} \theta\right\|_{H^{s}}^{2}+C_{l} \int_{0}^{M} \operatorname{E}_{t^{\prime} \in\left[0, t \wedge \sigma_{2, l}\right]} \sup \left\|\theta\left(t^{\prime}\right)\right\|_{H^{s}}^{2} \mathrm{~d} t,\right.
\end{aligned}
$$


where $C_{l}=C(1+l)$ for some $C>1$ large enough. Therefore, we arrive at

$$
\mathbb{E} \sup _{t \in\left[0, \sigma_{2, l} \wedge N\right]}\left\|T_{\varepsilon} \theta\right\|_{H^{s}}^{2}-2 \mathbb{E}\left\|T_{\varepsilon} \theta_{0}\right\|_{H^{s}}^{2} \leq C_{l} \int_{0}^{M} \mathbb{E} \sup _{t^{\prime} \in\left[0, t \wedge \sigma_{2, l}\right]}\left\|\theta\left(t^{\prime}\right)\right\|_{H^{s}}^{2} \mathrm{~d} t .
$$

Hence, one can send $\varepsilon \rightarrow 0$ and then use Grönwall's inequality to derive that for each $l, N \in \mathbb{N}$,

$$
\mathbb{E} \sup _{t \in\left[0, \sigma_{2, l} \wedge N\right]}\|\theta(t)\|_{H^{s}}^{2} \leq C \mathbb{E}\left\|\theta_{0}\right\|_{H^{s}}^{2} \exp \left(C_{l} N\right)<\infty
$$

which is (3.34). Hence, we obtain (3.14) and finish the proof.

\subsection{Further Examples}

Actually, the abstract framework for (1.1) can be applied to show the local existence theory to a broader class of fluid dynamics equations. For instance, consider the SALT surface quasi-geostrophic (SQG) equation:

$$
\left\{\begin{array}{l}
\mathrm{d} \theta+u \cdot \nabla \theta \mathrm{d} t+\sum_{k=1}^{\infty}\left(\xi_{k} \cdot \nabla \theta\right) \circ \mathrm{d} W_{k}=0, \quad x \in \mathbb{T}^{2}, \\
u=\mathcal{R}^{\perp} \theta
\end{array}\right.
$$

where $\mathcal{R}$ is the Riesz transform in $\mathbb{T}^{2}$, and $\left\{W_{t}^{k}\right\}_{k \in \mathbb{N}}$ is a sequence of standard 1-D independent Brownian motions. The deterministic version of (3.35) reduces to the SQG equation describing the dynamics of sharp fronts between masses of hot and cold air (cf. Constantin et al. 1994). The SQG equations have been studied intensively, and we cannot survey the vast research literature here. However, the stochastic version with transport noise as in (3.35) has not been studied yet as far as we know.

To apply Theorem 2.1 to (3.35) to get a local theory, we introduce some notations. For any real number $s, \Lambda^{s}=(-\Delta)^{s / 2}$ are defined by $\widehat{\Lambda^{s} f}(k)=|k|^{s} \widehat{f}(k)$. Then, we let

$$
\mathcal{X}^{s}=H^{s} \cap\left\{f: \int_{\mathbb{T}^{2}} f \mathrm{~d} x=0\right\} .
$$

We notice that with the mean-zero condition, $\mathcal{X}^{s}$ is Hilbert space for $s>0$ with inner product $(f, g) \mathcal{X}^{s}=\left(\Lambda^{s} f, \Lambda^{s} g\right)_{L^{2}}$ and homogeneous Sobolev norm $\|f\|_{\mathcal{X}^{s}}=$ $\left\|\Lambda^{s} f\right\|_{L^{2}}$. However, it can be shown that if $f \in \mathcal{X}^{s}$ for $s>0$, then, cf. Bahouri et al. (2011),

$$
\|f\|_{H^{s}} \lesssim\|f\|_{\mathcal{X}^{s}} \lesssim\|f\|_{H^{s}}
$$


Assumption (E) For all $s>1,\left\{\xi_{k}(x): \mathbb{T}^{2} \rightarrow \mathbb{R}^{2}\right\}_{k \in \mathbb{N}} \subset H^{s} \cap\left\{f \in H^{1}: \nabla \cdot f=0\right\}$ and $\sum_{k \in \mathbb{N}}\left\|\xi_{k}\right\|_{H^{s}}<\infty$.

Then, we have the following local results for (3.35):

Theorem 3.3 Let $s>4, \mathcal{S}=\left(\Omega, \mathcal{F}, \mathbb{P},\left\{\mathcal{F}_{t}\right\}_{t \geq 0}, \mathcal{W}\right)$ be a stochastic basis fixed in advance and $\mathcal{X}^{s}$ be given in (3.36). Let Assumption (E) hold true. If $\theta_{0} \in L^{2}\left(\Omega ; \mathcal{X}^{s}\right)$ is an $\mathcal{F}_{0}$-measurable random variable, then (3.35) has a local unique pathwise solution $\theta$ starting from $\theta_{0}$ such that

$$
\theta(\cdot \wedge \tau) \in L^{2}\left(\Omega ; C\left([0, \infty) ; \mathcal{X}^{s}\right)\right) .
$$

Moreover, the maximal solution $\left(\theta, \tau^{*}\right)$ to (3.35) satisfies

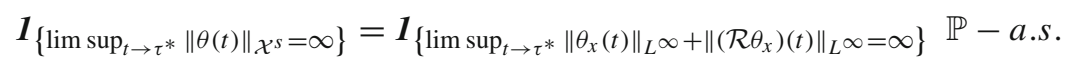

Proof We only give a very quick sketch. The approximation of (3.35) can be constructed as in the proof of Theorem 3.2. We only notice that if Assumption (E) is verified and $\theta_{0}$ has mean-zero, then the approximate solution $\theta_{\varepsilon}$ has also mean-zero. Recalling that $\mathbb{U}$ is fixed in advance to define (2.1), we take $\mathcal{X}=\mathcal{X}^{s}, \mathcal{Y}=\mathcal{X}^{s-1}$, $\mathcal{Z}=\mathcal{X}^{s-2}, \mathcal{V}=\mathcal{X}^{r}$ with $2<r<s-2$ and $T_{\varepsilon}=Q_{\varepsilon}=\tilde{J}_{\varepsilon}$, where $\tilde{J}_{\varepsilon}$ is given in (A.2). One can basically go along the lines as in the proof of Theorem 3.2 with using the $\Lambda^{s}$-version of Lemma A.4 (see also in Kato and Ponce 1988; Kenig et al. 1991) to estimate the nonlinear term. For the noise term, after writing it into the Itô form, one can use Lemma A.5 and (3.37) to estimate the corresponding two terms. For the sake of brevity, we omit the details.

Remark 3.5 If the relation $u=\mathcal{R}^{\perp} \theta$ in (3.35) is replaced by $u=\mathcal{R}^{\perp} \Lambda^{\alpha} u$ with $\alpha \in[-1,0],(3.35)$ becomes a SALT 2-D Euler- $\alpha$ model in vorticity form, which interpolates with the SALT 2-D Euler equations (Crisan and Lang 2019) $(\alpha=-1)$ and the SALT SQG equations $(\alpha=0)$. If $u=\mathcal{R}^{\perp} \mathcal{R}_{1} \theta$ in (3.35), then (3.35) is the SALT incompressible porous medium equation, where $\theta$ is now explained as the density of the incompressible fluid moving through a homogeneous porous domain. For the deterministic incompressible porous medium equation, we refer to Castro et al. (2009). Both of them with SALT noise $\sum_{k=1}^{\infty}\left(\xi_{k} \cdot \nabla \theta\right) \circ \mathrm{d} W_{k}$ have not been studied. Similar to Theorem 3.1, our general framework (ii) is also applicable to them.

Remark 3.6 It is worthwhile remarking that a new framework called LagrangianAveraged Stochastic Advection by Lie Transport (LA SALT) has been developed for a class of stochastic partial differential equations in Alonso-Orán et al. (2020) and Drivas et al. (2020). For LA SALT, the velocity field is randomly transported by white-noise vector fields as well as by its own average over realizations of this noise. For the even more general distribution-path dependent case of transport type equations, we refer to Ren et al. (2020). Generally speaking, the distribution of the solution is a global object on the path space, and it does not exist for explosive stochastic processes whose paths are killed at the life time. For a local theory of distribution dependent SDEs/SPDEs, we have to either consider the non-explosive setting or modify the "distribution" by 
a local notion (for example, conditional distribution given by solution does not blow up at present time). Here, we focus our attention to the abstract framework for SPDEs with SALT noise. The general case with LA SALT is left as future work.

Acknowledgements D. Alonso-Orán is deeply indebted to Antonio Córdoba for his helpful conversations about the theory of pseudo-differential operators. H. Tang benefited greatly from many insightful discussions with Professor Feng-Yu Wang. We sincerely thank the anonymous referees for the comments and suggestions which have led to an improvement in the article.

Funding Open access funding provided by University of Oslo (incl Oslo University Hospital).

Open Access This article is licensed under a Creative Commons Attribution 4.0 International License, which permits use, sharing, adaptation, distribution and reproduction in any medium or format, as long as you give appropriate credit to the original author(s) and the source, provide a link to the Creative Commons licence, and indicate if changes were made. The images or other third party material in this article are included in the article's Creative Commons licence, unless indicated otherwise in a credit line to the material. If material is not included in the article's Creative Commons licence and your intended use is not permitted by statutory regulation or exceeds the permitted use, you will need to obtain permission directly from the copyright holder. To view a copy of this licence, visit http://creativecommons.org/licenses/by/4.0/.

\section{A Auxiliary Results}

In this appendix, we formulate and prove some estimates employed in the proofs above. We start from mollifiers which can preserve periodicity. Let $j=j(x)$ be a Schwartz function such that $0 \leq \widehat{j}(\xi) \leq 1$ for all $\xi \in \mathbb{R}^{d}$ and $\widehat{j}(\xi)=1$ for any $|\xi| \leq 1$. Define for $\varepsilon \in(0,1)$ the mollifier

$$
J_{\varepsilon} g(x):=\left(j_{\varepsilon} \star g\right)(x),
$$

where $j_{\varepsilon}(x)=\frac{1}{\varepsilon} j\left(\frac{x}{\varepsilon}\right)$. The following operator $\tilde{J}_{\varepsilon}$ is also fundamental for the approximation and defined by

$$
\tilde{J}_{\varepsilon} g(x):=\left(1-\varepsilon^{2} \Delta\right)^{-1} g(x)=\sum_{k \in \mathbb{Z}^{d}}\left(1+\varepsilon^{2}|k|^{2}\right)^{-1} \widehat{g}(k) \mathrm{e}^{\mathrm{i} x \cdot k} .
$$

For any $u, v \in H^{s}, J_{\varepsilon}$ and $\tilde{J}_{\varepsilon}$ satisfy, cf. Tang $(2018,2020)$,

$$
\begin{aligned}
& \left\|u-J_{\varepsilon} u\right\|_{H^{r}} \sim o\left(\varepsilon^{s-r}\right), \quad r \leq s \\
& \left\|J_{\varepsilon} u\right\|_{H^{r}} \lesssim \varepsilon^{s-r}\|u\|_{H^{s}}, \quad r>s \\
& {\left[D^{s}, J_{\varepsilon}\right]=\left[D^{s}, \tilde{J}_{\varepsilon}\right]=0,} \\
& \left(J_{\varepsilon} u, v\right)_{L^{2}}=\left(u, J_{\varepsilon} v\right)_{L^{2}},\left(\tilde{J}_{\varepsilon} u, v\right)_{L^{2}}=\left(u, \tilde{J}_{\varepsilon} v\right)_{L^{2}},
\end{aligned}
$$

and

$$
\left\|J_{\varepsilon} u\right\|_{H^{s}},\left\|\tilde{J}_{\varepsilon} u\right\|_{H^{s}} \leq\|u\|_{H^{s}}, \quad\left\|J_{\varepsilon} u\right\|_{L^{\infty}} \lesssim\|u\|_{L^{\infty}}
$$


From the definition of the Hilbert transform $\mathcal{H}$ in (3.9), we have

$$
\left[D^{s}, \mathcal{H}\right]=\left[\partial_{x}, \mathcal{H}\right]=\left[J_{\varepsilon}, \mathcal{H}\right]=0,
$$

and for any $s \geq 0$,

$$
\|\mathcal{H} u\|_{H^{s}} \lesssim\|u\|_{H^{s}}
$$

A pseudo-differential operator $P(x, D)$ on the periodic torus $\mathbb{T}^{d}$ is an operator given by

$$
P(x, D) f(x)=\frac{1}{(2 \pi)^{d}} \sum_{k \in \mathbb{Z}^{d}} a(x, k) \mathrm{e}^{i x \cdot k} \widehat{f}(k),
$$

where $P(x, D)$ belongs to a certain class and $a(x, k)$ is called the symbol of $P(x, D)$. For $\rho, \delta \in[0,1], s \in \mathbb{R}$, we define the Hörmander class of symbols $S_{\rho, \delta}^{m}$ to be the set of all symbols $a: \mathbb{T}^{d} \times \mathbb{Z}^{d} \rightarrow \mathbb{C}$ such that $a(\cdot, k) \in C^{\infty}\left(\mathbb{T}^{d}\right)$ for all $k \in \mathbb{Z}^{d}$ and for all $\alpha, \beta \in \mathbb{N}^{d}$, there exists a constant $C=C(\alpha, \beta)>0$ such that

$$
\left|\Delta_{k}^{\alpha} \partial_{x}^{\beta} a(x, k)\right| \leq C\langle k\rangle^{s-\rho|\alpha|+\delta|\beta|},
$$

where $\langle k\rangle=\left(1+k^{2}\right)^{1 / 2}$ and for $g: \mathbb{Z}^{d} \rightarrow \mathbb{C}$,

$$
\Delta_{k}^{\alpha} g(k):=\sum_{\gamma \in \mathbb{N}^{d}, \gamma \leq \alpha}(-1)^{|\alpha-\gamma|}\left(\begin{array}{l}
\alpha \\
\gamma
\end{array}\right) g(k+\gamma)
$$

is the finite difference operator of order $\alpha$ with step size one in each of the coordinates of the frequency variable $k$. In such a case we say the associated operator $P(x, D)$ defined by (A.10) belongs to the class $\operatorname{OPS}_{\rho, \delta}^{s}$ (cf. Taylor 1991, page 8). Then, it is easy to check (see for example Taylor 1991 , pages $13 \& 22$ ) that $J_{\varepsilon}$ and $\tilde{J}_{\varepsilon}$ also satisfy

Lemma A.1 Let $J_{\varepsilon}, \tilde{J}_{\varepsilon}$ be defined as in (A.1) and (A.2), then the following properties hold true:

1. $J_{\varepsilon} \in O P S_{1,0}^{-\infty}, \tilde{J}_{\varepsilon} \in O P S_{1,0}^{-2}$ for every $\varepsilon \in(0,1)$;

2. $\left\{J_{\varepsilon}\right\}_{0<\varepsilon<1}$ and $\left\{\tilde{J}_{\varepsilon}\right\}_{0<\varepsilon<1}$ are bounded subsets of $O P S_{1,0}^{0}$;

3. If $p(x, D) \in O P S_{1,0}^{s}$, then $p(x, D) J_{\varepsilon} \in O P S_{1,0}^{-\infty}, p(x, D) \tilde{J}_{\varepsilon} \in O P S_{1,0}^{-\infty}$ for all $\varepsilon \in(0,1)$;

4. If $p(x, D) \in O P S_{1,0}^{s}$, then $\left\{p(x, D) J_{\varepsilon}\right\}_{0<\varepsilon<1} \subset O P S_{1,0}^{S}$ and $\left\{p(x, D) \tilde{J}_{\varepsilon}\right\}_{0<\varepsilon<1} \subset$ $O P S_{1,0}^{S}$ are bounded.

We also recall the following commutator estimates for two pseudo-differential operators. 
Lemma A.2 (Hörmander 1985; Taylor 1991) Let $\mathcal{P} \in O P S_{\rho, \delta}^{p}$ and $\mathcal{T} \in O P S_{\rho, \delta}^{q}$ with $p, q \in \mathbb{R}, 0 \leq \delta<\rho, \leq 1$ then

$$
[\mathcal{P}, \mathcal{T}] \in O P S_{\rho, \delta}^{p+q-(\rho-\delta)}
$$

Lemma A.3 (Tang 2020; Ren et al. 2020) Let $d \geq 1$ and $f, g: \mathbb{T}^{d} \rightarrow \mathbb{R}^{d}$ such that $g \in W^{1, \infty}$ and $f \in L^{2}$. Then, for some $C>0$,

$$
\left\|\left[\tilde{J}_{\varepsilon},(g \cdot \nabla)\right] f\right\|_{L^{2}} \leq C\|\nabla g\|_{L^{\infty}}\|f\|_{L^{2}} .
$$

Now we recall some useful estimates.

Lemma A.4 (Kato and Ponce 1988; Kenig et al. 1991) If $f, g \in H^{s} \cap W^{1, \infty}$ with $s>0$, then for $p, p_{i} \in(1, \infty)$ with $i=2,3$ and $\frac{1}{p}=\frac{1}{p_{1}}+\frac{1}{p_{2}}=\frac{1}{p_{3}}+\frac{1}{p_{4}}$, we have

$$
\left\|\left[D^{S}, f\right] g\right\|_{L^{p}} \leq C\left(\|\nabla f\|_{L^{p_{1}}}\left\|D^{s-1} g\right\|_{L^{p_{2}}}+\left\|D^{S} f\right\|_{L^{p_{3}}}\|g\|_{L^{p_{4}}}\right),
$$

and

$$
\left\|D^{S}(f g)\right\|_{L^{p}} \leq C\left(\|f\|_{L^{p_{1}}}\left\|D^{S} g\right\|_{L^{p_{2}}}+\left\|D^{s} f\right\|_{L^{p_{3}}}\|g\|_{L^{p_{4}}}\right)
$$

Lemma A.5 Let $s>\frac{d}{2}+1, f \in H^{s+2}$ be a scalar function, $\xi_{k}$ be a $d-D$ vector and $\mathcal{P} \in O P S_{1,0}^{S}$. Define

$$
\mathcal{L}_{\xi_{k}} f=\xi_{k} \cdot \nabla f+\left(\operatorname{div} \xi_{k}\right) f
$$

If Assumption (D) holds, then we have

$$
\left(\mathcal{P} \sum_{k=1}^{\infty} \mathcal{L}_{\xi_{k}}^{2} f, \mathcal{P} f\right)_{L^{2}}+\sum_{k=1}^{\infty}\left(\mathcal{P} \mathcal{L}_{\xi_{k}} f, \mathcal{P} \mathcal{L}_{\xi_{k}} f\right)_{L^{2}} \lesssim\|f\|_{H^{s}}^{2}
$$

Proof The essential part of the desired estimate lies in the following result in AlonsoOrán and Bethencourt de León (2020): Let $\mathcal{Q}$ be a first-order linear operator with smooth coefficients and $\mathcal{P} \in \mathrm{OPS}_{1,0}^{s}$. Then, $f \in H^{s}$ with $s>\frac{d}{2}+1$ we have that

$$
\left(\mathcal{P} \mathcal{Q}^{2} f, \mathcal{P} f\right)_{L^{2}}+(\mathcal{P} \mathcal{Q} f, \mathcal{P} \mathcal{Q} f)_{L^{2}} \lesssim\|f\|_{H^{s}}^{2}
$$

In particular, if we choose $\mathcal{Q}=\mathcal{L}_{\xi_{k}}$ we have that:

$$
\left(\mathcal{P} \mathcal{L}_{\xi_{k}}^{2} f, \mathcal{P} f\right)_{L^{2}}+\left(\mathcal{P} \mathcal{L}_{\xi_{k}} f, \mathcal{P} \mathcal{L}_{\xi_{k}} f\right)_{L^{2}} \lesssim\|f\|_{H^{s}}^{2}
$$

Since we want to calculate this estimate for $\sum_{k=1}^{\infty} \mathcal{L}_{\xi_{k}}^{2}$, we need to precise the constant of the right hand side of (A.12). To this end, mimicking the proof of Alonso-Orán and 
Bethencourt de León (2020) we can rewrite the left hand side of (A.12) as

$$
\begin{aligned}
(\mathcal{P} & \left.\mathcal{L}_{\xi_{k}}^{2} f, \mathcal{P} f\right)_{L^{2}}+\left(\mathcal{P} \mathcal{L}_{\xi_{k}} f, \mathcal{P} \mathcal{L}_{\xi_{k}} f\right)_{L^{2}} \\
= & \left(R_{2} f, \mathcal{P} f\right)_{L^{2}}+\left(R_{1} f, R_{1} f\right)_{L^{2}}+\left(\mathcal{P} f, E R_{1} f\right)_{L^{2}} \\
& -\frac{1}{2}\left(\mathcal{P} f, R_{0} \mathcal{P} f\right)_{L^{2}}+\frac{1}{2}\left(\mathcal{P} f, E^{2} \mathcal{P} f\right)_{L^{2}}+\left(R_{1} f, E \mathcal{P} f\right)_{L^{2}} \\
& :=\sum_{i=1}^{6} I_{i}
\end{aligned}
$$

where $E=\operatorname{div} \xi_{k} \in \operatorname{OPS}_{1,0}^{0}, R_{0}=\left[\mathcal{L}_{\xi_{k}}, E\right] \in \operatorname{OPS}_{1,0}^{1}, R_{1}=\left[\mathcal{P}, \mathcal{L}_{\xi_{k}}\right]$ and $R_{2}=$ $\left[R_{1}, \mathcal{L}_{\xi_{k}}\right]$. By Lemma A.2, we have

$$
R_{1}, R_{2},\left[R_{1}, \nabla\right] \in \mathrm{OPS}_{1,0}^{s}
$$

To derive (A.11) we will invoke the following commutator estimates (see Taylor 1991, (3.6.1) and (3.6.2)):

- If $P \in \mathrm{OPS}_{1,0}^{s}, s>0$, then there is a $C>0$ such that

$$
\|P(g u)-g P u\|_{L^{2}} \leq C\left(\|g\|_{W^{1, \infty}}\|u\|_{H^{s-1}}+\|g\|_{H^{s}}\|u\|_{L^{\infty}}\right) .
$$

- If $P \in \mathrm{OPS}_{1,0}^{1}$, then there is a $C>0$ such that

$$
\|P(g u)-g P u\|_{L^{2}} \leq C\|g\|_{W^{1, \infty}}\|u\|_{L^{2}} .
$$

For $I_{1}$, we have that

$$
\begin{aligned}
\left|I_{1}\right| & \leq\left\|R_{2} f\right\|_{L^{2}}\|P f\|_{L^{2}} \\
& \leq\left\|\left[R_{1}, \mathcal{L}_{\xi_{k}}\right] f\right\|_{L^{2}}\|f\|_{H^{s}} \\
& =\left(\left\|\left[R_{1}, \xi_{k} \cdot \nabla\right] f\right\|_{L^{2}}+\left\|\left[R_{1}, \operatorname{div} \xi_{k}\right] f\right\|_{L^{2}}\right)\|f\|_{H^{s}} \\
& =\left(\left\|\left[R_{1}, \xi_{k} \cdot\right] \nabla f\right\|_{L^{2}}+\left\|\xi_{k} \cdot\left[R_{1}, \nabla\right] f\right\|_{L^{2}}+\left\|\left[R_{1}, \operatorname{div} \xi_{k}\right] f\right\|_{L^{2}}\right)\|f\|_{H^{s}} \\
& =\left(I_{1,1}+I_{1,2}+I_{1,3}\right)\|f\|_{H^{s}}
\end{aligned}
$$

Applying (A.13) with $P=R_{1}, g=\xi_{k}, u=\nabla f$, and using $H^{s} \hookrightarrow W^{1, \infty}$, we arrive at

$$
\left|I_{1,1}\right| \leq\left\|\xi_{k}\right\|_{W^{1, \infty}}\|\nabla f\|_{H^{s-1}}+\left\|\xi_{k}\right\|_{H^{s}}\|\nabla f\|_{L^{\infty}} \leq\left\|\xi_{k}\right\|_{H^{s}}\|f\|_{H^{s}}
$$

For the second term, we have

$$
\left|I_{1,2}\right|=\left\|\xi_{k} \cdot\left[R_{1}, \nabla\right] f\right\|_{L^{2}} \leq\left\|\xi_{k}\right\|_{L^{\infty}}\left\|\left[R_{1}, \nabla\right] f\right\|_{L^{2}} \leq\left\|\xi_{k}\right\|_{H^{s}}\|f\|_{H^{s}}
$$


Applying (A.13) with $P=R_{1}, g=\operatorname{div} \xi_{k}$ and $u=f$ yields

$$
\left|I_{1,3}\right| \leq\left\|\operatorname{div} \xi_{k}\right\|_{W^{1, \infty}}\|f\|_{H^{s-1}}+\left\|\operatorname{div} \xi_{k}\right\|_{H^{s}}\|f\|_{L^{\infty}} \leq\left\|\xi_{k}\right\|_{H^{s+1}}\|f\|_{H^{s}}
$$

Hence, we have show that

$$
\left|I_{1}\right| \leq C\left\|\xi_{k}\right\|_{H^{s+1}}\|f\|_{H^{s}}^{2}
$$

Repeat the above procedure as we estimate $\left\|R_{2} f\right\|_{L^{2}}=\left\|\left[R_{1}, \mathcal{L}_{\xi_{k}}\right] f\right\|_{L^{2}}$ with replacing $R_{1}$ by $\mathcal{P}$, we have

$$
\begin{aligned}
\left|I_{2}\right| & \leq\left\|R_{1} f\right\|_{L^{2}}^{2} \leq\left\|\left[\mathcal{P}, \mathcal{L}_{\xi_{k}}\right] f\right\|_{L^{2}}^{2} \\
& =\left(\left\|\left[\mathcal{P}, \xi_{k} \cdot \nabla\right] f\right\|_{L^{2}}+\left\|\left[\mathcal{P}, \operatorname{div} \xi_{k}\right] f\right\|_{L^{2}}\right)^{2} \\
& =\left(\left\|\left[\mathcal{P}, \xi_{k} \cdot\right] \nabla f\right\|_{L^{2}}+\left\|\xi_{k} \cdot[\mathcal{P}, \nabla] f\right\|_{L^{2}}+\left\|\left[\mathcal{P}, \operatorname{div} \xi_{k}\right] f\right\|_{L^{2}}\right)^{2} \\
& \leq\left\|\xi_{k}\right\|_{H^{s+1}}^{2}\|f\|_{H^{s}}^{2},
\end{aligned}
$$

For the third term, using the Cauchy-Schwarz inequality and the fact that $E=\operatorname{div} \xi_{k} \in$ $\mathrm{OPS}_{1,0}^{1}$ gives rise to

$$
\left|I_{3}\right|=\left(\mathcal{P} f, E R_{1} f\right)_{L^{2}} \leq\|\mathcal{P} f\|_{L^{2}}\left\|\operatorname{div} \xi_{k} R_{1} f\right\|_{L^{2}} \leq\left\|\operatorname{div} \xi_{k}\right\|_{L^{\infty}}\|f\|_{H^{s}}^{2} .
$$

Similarly,

$$
\begin{aligned}
\left|I_{5}+I_{6}\right| & =\left|\frac{1}{2}\left(\mathcal{P} f, E^{2} \mathcal{P} f\right)_{L^{2}}+\left(R_{1} f, E \mathcal{P} f\right)_{L^{2}}\right| \\
& \leq C\left(\left\|\operatorname{div} \xi_{k}\right\|_{L^{\infty}}^{2}\|\mathcal{P} f\|_{L^{2}}^{2}+\left\|R_{1} f\right\|_{L^{2}}\left\|\operatorname{div} \xi_{k}\right\|_{L^{\infty}}\|\mathcal{P} f\|_{L^{2}}\right) \\
& \leq C\left(\left\|\operatorname{div} \xi_{k}\right\|_{L^{\infty}}+\left\|\operatorname{div} \xi_{k}\right\|_{L^{\infty}}^{2}\right)\|f\|_{H^{2}}^{2} .
\end{aligned}
$$

For $I_{4}$, we notice that $\mathcal{L}_{\xi_{k}} \in \operatorname{OPS}_{1,0}^{1}$. Hence, it follows from (A.14) with $P=\mathcal{L}_{\xi_{k}}$, $g=\operatorname{div} \xi_{k}$ and $u=\mathcal{P} f$ that

$$
\begin{aligned}
\left|I_{4}\right| & \leq C\|\mathcal{P} f\|_{L^{2}}\left\|\left[\mathcal{L}_{\xi_{k}}, \operatorname{div} \xi_{k}\right] \mathcal{P} f\right\|_{L^{2}} \leq C\|\mathcal{P} f\|_{L^{2}}\left\|\operatorname{div} \xi_{k}\right\|_{W^{1, \infty}}\|\mathcal{P} f\|_{L^{2}} \\
& \leq C\left\|\xi_{k}\right\|_{H^{s+1}}\|f\|_{H^{s}}^{2}
\end{aligned}
$$

Gathering all the above estimates implies that for some $C>0$,

$$
\left(\mathcal{P} \mathcal{L}_{\xi_{k}}^{2} f, \mathcal{P} f\right)_{L^{2}}+\left(\mathcal{P} \mathcal{L}_{\xi_{k}} f, \mathcal{P} \mathcal{L}_{\xi_{k}} f\right)_{L^{2}} \leq C\left(\left\|\xi_{k}\right\|_{H^{s+1}}^{2}+\left\|\xi_{k}\right\|_{H^{s+1}}\right)\|f\|_{H^{s}}^{2}
$$

Using Assumption (D) to the above estimates, we obtain (A.11).

We conclude this appendix with some useful tools in stochastic analysis. 
Lemma A.6 (Prokhorov Theorem, Da Prato and Zabczyk (2014)) Let $\mathbb{X}$ be a complete and separable metric space. A sequence of measures $\left\{\mu_{n}\right\} \subset \mathscr{P}(\mathbb{X})$ is tight if and only if it is relatively compact, i.e., there is a subsequence $\left\{\mu_{n_{k}}\right\}$ converging to a probability measure $\mu$ weakly.

Lemma A.7 (Skorokhod Theorem, Da Prato and Zabczyk (2014)) Let $\mathbb{X}$ be a complete and separable metric space. For an arbitrary sequence $\left\{\mu_{n}\right\} \subset \mathscr{P}(\mathbb{X})$ such that $\left\{\mu_{n}\right\}$ is tight on $(\mathbb{X}, \mathcal{B}(\mathbb{X}))$, there exists a subsequence $\left\{\mu_{n_{k}}\right\}$ converging weakly to a probability measure $\mu$, and a probability space $(\Omega, \mathcal{F}, \mathbb{P})$ with $\mathbb{X}$-valued Borel measurable random variables $x_{n}$ and $x$, such that $\mu_{n}$ is the distribution of $x_{n}, \mu$ is the distribution of $x$, and $x_{n} \stackrel{n \rightarrow \infty}{\longrightarrow} x \mathbb{P}-$ a.s.

Lemma A.8 (Breit et al. 2018; Debussche et al. 2011) Let $(\Omega, \mathcal{F}, \mathbb{P})$ be a complete probability space and $\mathbb{X}$ be a separable Hilbert space and let $\mathcal{S}_{n}=$ $\left(\Omega, \mathcal{F},\left\{\mathcal{F}_{t}^{n}\right\}_{t \geq 0}, \mathbb{P}, \mathcal{W}_{n}\right)$ be a sequence of stochastic bases such that for each $n \geq 1, \mathcal{W}^{n}$ is cylindrical Brownian motion (over $\mathbb{U}$ with the canonical embedding $\mathbb{U} \hookrightarrow \mathbb{U}_{0}$ being Hilbert-Schmidt) with respect to $\left\{\mathcal{F}_{t}^{n}\right\}_{t \geq 0}$. Let $G_{n}$ be an $\mathcal{F}_{t}^{n}$ predictable process ranging in $\mathcal{L}_{2}(\mathbb{U} ; \mathbb{X})$. Finally consider $\mathcal{S}=\left(\Omega, \mathcal{F}, \mathbb{P},\left\{\mathcal{F}_{t}\right\}_{t \geq 0}, \mathcal{W}\right)$ and $G \in L^{2}\left(0, T ; \mathcal{L}_{2}(\mathbb{U} ; \mathbb{X})\right)$, which is $\mathcal{F}_{t}$ predictable. Suppose that we have the following convergence in probability:

$$
\mathcal{W}_{n} \rightarrow \mathcal{W} \text { in } C\left([0, T] ; \mathbb{U}_{0}\right) \text { and } G_{n} \rightarrow G \text { in } L^{2}\left(0, T ; \mathcal{L}_{2}(\mathbb{U} ; \mathbb{X})\right)
$$

Then,

$$
\int_{0}^{\cdot} G_{n} \mathrm{~d} \mathcal{W}_{n} \rightarrow \int_{0}^{\cdot} G \mathrm{~d} \mathcal{W} \quad \text { in } L^{2}(0, T ; \mathbb{X}) \text { in probability. }
$$

Lemma A.9 (Gyöngy-Krylov Lemma, Gyöngy and Krylov (1996)) Let $\mathbb{X}$ be a Polish space equipped with the Borel sigma-algebra $\mathcal{B}(\mathbb{X})$. Let $\left\{Y_{j}\right\}_{j \geq 0}$ be a sequence of $\mathbb{X}$-valued random variables. Let

$$
\mu_{j, l}(\cdot):=\mathbb{P}\left(\left(Y_{j}, Y_{l}\right) \in \cdot\right) \quad \forall \cdot \in \mathcal{B}(\mathbb{X} \times \mathbb{X})
$$

Then, $\left\{Y_{j}\right\}_{j \geq 0}$ converges in probability if and only if for every subsequence of $\left\{\mu_{j_{k}, l_{k}}\right\}_{k \geq 0}$, there exists a further subsequence which weakly converges to some $\mu \in \mathscr{P}(\mathbb{X} \times \mathbb{X})$ satisfying

$$
\mu(\{(u, v) \in \mathbb{X} \times \mathbb{X}, u=v\})=1 .
$$

\section{References}

Albeverio, S., Brzeźniak, Z., Daletskii, A.: Stochastic Camassa-Holm equation with convection type noise. J. Differ. Equ. 276(5), 404-432 (2021)

Alonso-Orán, D., Bethencourt de León, A.: On the well-posedness of stochastic Boussinesq equations with transport noise. J. Nonlinear Sci. 30(1), 175-224 (2020) 
Alonso-Orán, D., Bethencourt de León, A., Takao, S.: The Burgers' equation with stochastic transport: shock formation, local and global existence of smooth solutions. NoDEA Nonlinear Differ. Equ. Appl. 26(6), No. 57, 33 (2019)

Alonso-Orán, D., Bethencourt de León, A., Holm, D.D., Takao, S.: Modelling the climate and weather of a 2-D Lagrangian-averaged Euler-Boussinesq equation with transport noise. J. Stat. Phys. 179, 1267-1303 (2020)

Bae, H., Granero-Belinchón, R.: Global existence for some transport equations with nonlocal velocity. Adv. Math. 269, 197-219 (2015)

Bahouri, H., Chemin, J., Danchin, R.: Fourier analysis and nonlinear partial differential equations. Grundlehren der Mathematischen Wissenschaften, vol. 343. Springer, Heidelberg (2011)

Baker, G.R., Li, X., Morlet, A.C.: Analytic structure of 1D-transport equations with nonlocal fluxes. Physica D 91, 349-375 (1996)

Bendall, T., Cotter, C., Holm, D.D.: Perspectives on the Formation of Peakons in the Stochastic CamassaHolm Equation. arXiv:1910.03018v (2019)

Bensoussan, A.: Stochastic Navier-Stokes equations. Acta Appl. Math. 38(3), 267-304 (1995)

Berner, J., Jung, T., Palmer, T.N.: Systematic model error: The impact of increased horizontal resolution versus improved stochastic and deterministic parameterizations. Journal of Climate 25(14), 4946-4962 (2012)

Breit, D., Feireisl, E., Hofmanová, M.: Stochastically forced compressible fluid flows. De Gruyter Series in Applied and Numerical Mathematics, vol. 3. De Gruyter, Berlin (2018)

Bressan, A., Constantin, A.: Global conservative solutions of the Camassa-Holm equation. Arch. Ration. Mech. Anal. 183(2), 215-239 (2007)

Camassa, R., Holm, D.D.: An integrable shallow water equation with peaked solitons. Phys. Rev. Lett. 71(11), 1661-1664 (1993)

Castro, A., Córdoba, D., Gancedo, F., Orive, R.: Incompressible flow in porous media with fractional diffusion. Nonlinearity 22(8), 1791-1815 (2009)

Chen, Y., Gao, H., Guo, B.: Well-posedness for stochastic Camassa-Holm equation. J. Differential Equations 253(8), 2353-2379 (2012)

Constantin, A., Escher, J.: Wave breaking for nonlinear nonlocal shallow water equations. Acta Math. 181(2), 229-243 (1998a)

Constantin, A., Escher, J.: Well-posedness, global existence, and blowup phenomena for a periodic quasilinear hyperbolic equation. Commun. Pure Appl. Math. 51(5), 475-504 (1998b)

Constantin, P., Majda, A., Tabak, E.: Formation of strong fronts in the 2-D quasi-geostrophic thermal active scalar. Nonlinearity 7, 1495-1533 (1994)

Córdoba, A., Córdoba, D., Fontelos, M.A.: Formation of singularities for a transport equation with nonlocal velocity. Ann. of Math. 162(3), 1377-1389 (2005)

Cotter, C., Gottwald, G., Holm, D.D.: Stochastic partial differential fluid equations as a diffusive limit of deterministic lagrangian multi-time dynamics. Proc. R. Soc. A 473(2205), 20170388 (2017)

Cotter, C., Crisan, D., Holm, D.D., Pan, W., Shevchenko, I.: A Particle Filter for Stochastic Advection by Lie Transport (SALT): a case study for the damped and forced incompressible 2-D Euler equation. arXiv:1907.11884 [stat.AP] (2019)

Crisan, D., Holm, D.: Wave breaking for the stochastic Camassa-Holm equation. Physica D: Nonlinear Phenomena 376, 138-143 (2018)

Crisan, D., Lang, O.: Well-posedness for a stochastic 2-D Euler equation with transport noise. arXiv:1907.00451 (2019)

Crisan, D., Flandoli, F., Holm, D.D.: Solution Properties of a 3D Stochastic Euler Fluid Equation. J. Nonlinear Sci. 29(3), 813-870 (2019)

Da Prato, G., Zabczyk, J.: Stochastic Equations in Infinite Dimensions, of Encyclopedia of Mathematics and Its Applications, vol. 152, 2nd edn. Cambridge University Press, Cambridge (2014)

Debussche, A., Glatt-Holtz, N.E., Temam, R.: Local martingale and pathwise solutions for an abstract fluids model. Phys. D 240(14-15), 1123-1144 (2011)

Dong, H.: Well-posedness for a transport equation with nonlocal velocity. J. Funct. Anal. 255(11), 30703097 (2008)

Drivas, T., Holm, D.D., Leahy, J.M.: Lagrangian Averaged Stochastic Advection by Lie Transport for Fluids. Journal of Statistical Physics 179, 1304-1342 (2020)

Fedrizzi, E., Flandoli, F.: Noise prevents singularities in linear transport equations. J. Funct. Anal. 264(6), 1329-1354 (2013) 
Flandoli, F.: Random perturbation of PDEs and fluid dynamic models, volume 2015 of Lecture Notes in Mathematics. Springer, Heidelberg, (2011). Lectures from the 40th Probability Summer School held in Saint-Flour, 2010, École d'Été de Probabilités de Saint-Flour. [Saint-Flour Probability Summer School]

Flandoli, F., Luo, D.: Euler-Lagrangian approach to 3-D stochastic Euler equations. J. Geom. Mech. 11(2), 153-165 (2019)

Flandoli, F., Gubinelli, M., Priola, E.: Well-posedness of the transport equation by stochastic perturbation. Invent. Math. 180(1), 1-53 (2010)

Fuchssteiner, B., Fokas, A.S.: Symplectic structures, their Bäcklund transformations and hereditary symmetries. Phys. D 4(1), 47-66 (1981)

Gawarecki, L., Mandrekar, V.: Stochastic differential equations in infinite dimensions with applications to stochastic partial differential equations Probability and its Applications (New York). Springer, Heidelberg, (2011)

Glatt-Holtz, N., Vicol, V.: Local and global existence of smooth solutions for the stochastic Euler equations with multiplicative noise. Ann. Probab. 42(1), 80-145 (2014)

Glatt-Holtz, N., Ziane, M.: Strong pathwise solutions of the stochastic Navier-Stokes system. Adv. Differ. Equ. 14(5-6), 567-600 (2009)

Gyöngy, I., Krylov, N.: Existence of strong solutions for Itô's stochastic equations via approximations. Probab. Theory Relat. Fields 105(2), 143-158 (1996)

Holden, H., Raynaud, X.: Global conservative solutions of the Camassa-Holm equation-a Lagrangian point of view. Comm. Partial Differential Equations 32(10-12), 1511-1549 (2007)

Holm, D.D.: Variational principles for stochastic fluid dynamics. Proc. A. 471(2176), 963 (2015)

Holm, D.D., Luesink, E.: Stochastic wave-current interaction in thermal shallow water dynamics. Journal of Nonlinear Science 3(21),(2021)

Hörmander, L.: The analysis of linear partial differential operators. III, volume 274 of Grundlehren der Mathematischen Wissenschaften [Fundamental Principles of Mathematical Sciences]. Springer, Berlin, (1985). Pseudodifferential operators

Kallianpur, G., Xiong, J.: Stochastic differential equations in infinite-dimensional spaces. 26:vi+342, expanded version of the lectures delivered as part of the 1993 Barrett Lectures at the University of Tennessee, Knoxville, TN, March 25-27, 1993. With a foreword by Balram S, Rajput and Jan Rosinski (1995)

Karczewska, A.: Stochastic integral with respect to cylindrical Wiener process. Ann. Univ. Mariae CurieSkłodowska Sect. A 52(2), 79-93 (1998)

Kato, T., Ponce, G.: Commutator estimates and the Euler and Navier-Stokes equations. Commun. Pure Appl. Math. 41(7), 891-907 (1988)

Kenig, C.E., Ponce, G., Vega, L.: Well-posedness of the initial value problem for the Korteweg-de Vries equation. J. Amer. Math. Soc. 4(2), 323-347 (1991)

Krylov, N.V., Rozovskiĭ, B.L.: Stochastic evolution equations. In Current problems in mathematics, Vol. 14 (Russian), pages 71-147, 256. Akad. Nauk SSSR, Vsesoyuz. Inst. Nauchn. i Tekhn. Informatsii, Moscow (1979)

Leha, G., Ritter, G.: On solutions to stochastic differential equations with discontinuous drift in Hilbert space. Math. Ann. 270(1), 109-123 (1985)

Leslie, D., Quarini, G.: The application of turbulence theory to the formulation of subgrid modelling procedures. Journal of Fluid Mechanics 91, 65-91 (1979)

Li, J., Liu, H., Tang, H.: Stochastic MHD equations with fractional kinematic dissipation and partial magnetic diffusion in $\mathbb{R}^{2}$. Stochastic Process. Appl. 135, 139-182 (2021)

Pardoux, E.: Sur des équations aux dérivées partielles stochastiques monotones. C. R. Acad. Sci. Paris Sér. A-B 275, A101-A103 (1972)

Prévôt, C., Röckner, M.: A concise course on stochastic partial differential equations. Lecture Notes in Mathematics, vol. 1905. Springer, Berlin (2007)

Ren, P., Tang, H., Wang, F.-Y.: Distribution-path dependent nonlinear SPDEs with application to stochastic transport type equations. arXiv:2007.09188 (2020)

Rohde, C., Tang, H.: On a stochastic Camassa-Holm type equation with higher order nonlinearities. J. Dyn. Diff. Equat. (2020). https://doi.org/10.1007/s10884-020-09872-1

Rohde, C., Tang, H.: On the stochastic Dullin-Gottwald-Holm equation: global existence and wave-breaking phenomena. NoDEA Nonlinear Differential Equations Appl. 28(1), 34 (2021) 
Tang, H.: On the pathwise solutions to the Camassa-Holm equation with multiplicative noise. SIAM J. Math. Anal. 50(1), 1322-1366 (2018)

Tang, H.: Noise effects on dependence on initial data and blow-up for stochastic Euler-Poincaré equations. arXiv:2002.08719 (2020)

Taylor, M.E.: Pseudodifferential operators and nonlinear PDE. Progress in Mathematics, vol. 100. Birkhäuser Boston Inc, Boston, MA (1991)

Temam, R.: Navier-Stokes equations. Theory and numerical analysis. North-Holland Publishing Co., Amsterdam-New York-Oxford, (1977). Studies in Mathematics and its Applications, Vol. 2

Zidikheri, M., Frederiksen, J.: Stochastic subgrid-scale modelling for non-equilibrium geophysical flows Philosophical Transactions of the Royal Society A: Mathematica. Physical and Engineering Sciences 368, 145-160 (2010)

Publisher's Note Springer Nature remains neutral with regard to jurisdictional claims in published maps and institutional affiliations. 